\title{
A 'Darboux Theorem' for shifted symplectic structures on derived Artin stacks, with applications
}

\author{
Oren Ben-Bassat, Christopher Brav, Vittoria Bussi \\ and Dominic Joyce
}

\begin{abstract}
This is the fifth in a series of papers [15, 4, 3, 3] on the ' $k$-shifted symplectic derived algebraic geometry' of Pantev, Toën, Vaquié and Vezzosi [30. This paper extends the results of [4, 3], [5] from (derived) schemes to (derived) Artin stacks. We prove four main results:
\end{abstract}

(a) If $\left(\boldsymbol{X}, \omega_{\boldsymbol{X}}\right)$ is a $k$-shifted symplectic derived Artin stack for $k<0$ in the sense of 30, then near each $x \in \boldsymbol{X}$ we can find a 'minimal' smooth atlas $\boldsymbol{\varphi}: \boldsymbol{U} \rightarrow \boldsymbol{X}$ with $\boldsymbol{U}$ an affine derived scheme, such that $\left(\boldsymbol{U}, \boldsymbol{\varphi}^{*}\left(\omega_{\boldsymbol{X}}\right)\right)$ may be written explicitly in coordinates in a standard 'Darboux form'.

(b) If $\left(\boldsymbol{X}, \omega_{\boldsymbol{X}}\right)$ is a -1 -shifted symplectic derived Artin stack and $X=$ $t_{0}(\boldsymbol{X})$ the corresponding classical Artin stack, then $X$ extends naturally to a 'd-critical stack' $(X, s)$ in the sense of [15].

(c) If $(X, s)$ is an oriented d-critical stack, we can define a natural perverse sheaf $\check{P}_{X, s}^{\bullet}$ on $X$, such that whenever $T$ is a scheme and $t: T \rightarrow X$ is smooth of relative dimension $n$, then $T$ is locally modelled on a critical locus $\operatorname{Crit}\left(f: U \rightarrow \mathbb{A}^{1}\right)$ for $U$ a smooth scheme, and $t^{*}\left(\check{P}_{X, s}^{\bullet}\right)[n]$ is locally modelled on the perverse sheaf of vanishing cycles $\mathcal{P} \mathcal{V}_{U, f}^{\bullet}$ of $f$.

(d) If $(X, s)$ is a finite type oriented d-critical stack, we can define a natural motive $M F_{X, s}$ in a certain ring of motives $\overline{\mathcal{M}}_{X}^{\text {st, }, \hat{\mu}}$ on $X$, such that whenever $T$ is a finite type scheme and $t: T \rightarrow X$ is smooth of dimension $n$, then $T$ is locally modelled on a critical locus $\operatorname{Crit}\left(f: U \rightarrow \mathbb{A}^{1}\right)$ for $U$ a smooth scheme, and $\mathbb{L}^{-n / 2} \odot t^{*}\left(M F_{X, s}\right)$ is locally modelled on the motivic vanishing cycle $M F_{U, f}^{\text {mot, } \phi}$ of $f$ in $\overline{\mathcal{M}}_{T}^{\text {st, } \hat{\mu}}$.

Our results will have applications to categorified and motivic extensions of Donaldson-Thomas theory of Calabi-Yau 3-folds.

\section{Contents}

1 Introduction 2

2 Local models for atlases of shifted symplectic derived stacks 6

2.1 Derived algebraic geometry . . . . . . . . . . 6 
2.2 Shifted symplectic derived schemes and derived stacks . . . . . . 7

2.3 'Standard form' affine derived schemes . . . . . . . . . . . . . 7

2.4 'Darboux form' shifted symplectic derived schemes . . . . . . . . 10

2.5 'Standard form' atlases for derived stacks . . . . . . . . . . . . . 12

2.6 'Darboux form' atlases for shifted symplectic derived stacks . . . 14

2.7 Comparing 'Darboux form' atlases on overlaps . . . . . . . . 19

3 A truncation functor to d-critical stacks

3.1 Algebraic d-critical loci, the $\mathbb{K}$-scheme case . . . . . . . . . 20

3.2 Extension to Artin stacks, and d-critical stacks . . . . . . . . . . 24

3.3 From -1-shifted symplectic derived stacks to d-critical stacks . . 28

3.4 Proof of Theorem 3.18 . . . . . . . . . . . . . . . . . . . 29

4 Perverse sheaves on d-critical stacks 32

4.1 Perverse sheaves on schemes . . . . . . . . . . . . . . 32

4.2 Perverse sheaves on d-critical loci . . . . . . . . . . . . . . . . . . . . . . . . . . 35

4.3 Perverse sheaves on Artin stacks . . . . . . . . . . . . . . . . . 39

4.4 The main result . . . . . . . . . . . . . . . . . . 41

5 Motives on d-critical stacks

5.1 Rings of motives on $\mathbb{K}$-schemes . . . . . . . . . . . . . . 44

5.2 Motivic vanishing cycles, and d-critical loci . . . . . . . . . . . . 49

5.3 Rings of motives over Artin stacks . . . . . . . . . . . . . . . 51

5.4 The main result . . . . . . . . . . . . . . . . . 55

5.5 Proof of Theorem 5.14 ................. . . . . . . . . .

References $\quad 59$

\section{Introduction}

This is the fifth in a series of papers [15, 4, 3, 3, 5] on the subject of the ' $k$-shifted symplectic derived algebraic geometry' of Pantev, Toën, Vaquié and Vezzosi [30, and its applications to generalizations of Donaldson-Thomas theory of Calabi-Yau 3-folds, and to complex and algebraic symplectic geometry.

Pantev et al. 30] defined notions of $k$-shifted symplectic derived schemes and stacks $(\boldsymbol{X}, \omega)$, a new geometric structure on derived schemes and derived stacks $\boldsymbol{X}$ in the sense of Toën and Vezzosi 32.33. They proved that any derived moduli stack $\mathcal{M}$ of (complexes of) coherent sheaves on a Calabi-Yau $m$-fold $Y$ carries a $(2-m)$-shifted symplectic structure.

We are particularly interested in Calabi-Yau 3-folds, in which case $k=-1$. Pantev et al. [30] also proved that the derived critical locus $\operatorname{Crit}\left(f: U \rightarrow \mathbb{A}^{1}\right)$ of a regular function $f$ on a smooth $\mathbb{K}$-scheme $U$ is -1-shifted symplectic, and that the derived intersection $L \cap M$ of two algebraic Lagrangian submanifolds $L, M$ in an algebraic symplectic manifold $(S, \omega)$ is -1-shifted symplectic.

The first paper Joyce [15] in our series defined and studied 'algebraic dcritical loci' $(X, s)$, a classical $\mathbb{K}$-scheme $X$ with a geometric structure $s$ which 
records information on how $X$ may Zariski locally be written as a classical critical locus $\operatorname{Crit}\left(f: U \rightarrow \mathbb{A}^{1}\right)$ of a regular function $f$ on a smooth $\mathbb{K}$-scheme $U$. It also discussed 'd-critical stacks' $(X, s)$, a generalization to Artin $\mathbb{K}$-stacks.

The second paper by Bussi, Brav and Joyce [4] proved a 'Darboux Theorem' for the $k$-shifted symplectic derived schemes $(\boldsymbol{X}, \omega)$ of 30 when $k<0$, writing $(\boldsymbol{X}, \omega)$ Zariski locally in a standard form, and defined a truncation functor from -1 -shifted symplectic derived schemes $(\boldsymbol{X}, \omega)$ to algebraic d-critical loci $(X, s)$. By [30, this implies that moduli schemes $\mathcal{M}$ of simple (complexes of) coherent sheaves on a Calabi-Yau 3-fold $Y$ can be made into d-critical loci $(\mathcal{M}, s)$.

The third paper by Bussi, Brav, Dupont, Joyce and Szendrői [3] proves that if $(X, s)$ is an algebraic d-critical locus with an 'orientation', then one can define a natural perverse sheaf $P_{X, s}^{\bullet}$, a $\mathscr{D}$-module $D_{X, s}$, and (over $\mathbb{K}=\mathbb{C}$ ) a mixed Hodge module $M_{X, s}$ over $X$, such that if $(X, s)$ is locally modelled on $\operatorname{Crit}\left(f: U \rightarrow \mathbb{A}^{1}\right)$ then $P_{X, s}^{\bullet}$ is locally modelled on the perverse sheaf of vanishing cycles $\mathcal{P} \mathcal{V}_{U, f}^{\bullet}$ of $f$, and similarly for $D_{X, s}, M_{X, s}$. We hope to apply this to the categorification of Donaldson-Thomas theory of Calabi-Yau 3-folds, as in Kontsevich and Soibelman [19.

The fourth paper by Bussi, Joyce and Meinhardt [5] proves that if $(X, s)$ is a finite type, oriented algebraic d-critical locus then one can define a natural motive $M F_{X, s}$ in a ring of motives $\overline{\mathcal{M}}_{X}^{\hat{\mu}}$ on $X$, such that if $(X, s)$ is locally modelled on $\operatorname{Crit}\left(f: U \rightarrow \mathbb{A}^{1}\right)$ then $M F_{X, s}$ is locally modelled on the 'motivic vanishing cycle' $M F_{U, f}^{\text {mot, } \phi}$ of $f$. We hope to apply this to motivic DonaldsonThomas invariants of Calabi-Yau 3-folds, as in Kontsevich and Soibelman [18].

The goal of this paper is to extend the results of [4, [3], [5] from $\mathbb{K}$-schemes to Artin $\mathbb{K}$-stacks, using the notion of d-critical stack from [15]. The next four theorems summarize the main results of sections 2 5 below, respectively:

Theorem 1.1. Let $\mathbb{K}$ be an algebraically closed field of characteristic zero, $\left(\boldsymbol{X}, \omega_{\boldsymbol{X}}\right)$ a $k$-shifted symplectic derived Artin $\mathbb{K}$-stack as in 30 for $k<0$, and $p \in \boldsymbol{X}(\mathbb{K})$ be a $\mathbb{K}$-point of $\boldsymbol{X}$. Then we can construct the following data:

(a) Affine derived $\mathbb{K}$-schemes $\boldsymbol{U}=\operatorname{Spec} A, \boldsymbol{V}=\operatorname{Spec} B$, where $A, B$ are commutative differential graded $\mathbb{K}$-algebras (cdgas) in degrees $\leqslant 0$, of an explicit 'standard form' defined in $\$ 2.3$.

(b) A morphism of derived stacks $\boldsymbol{\varphi}: \boldsymbol{U}=\operatorname{Spec} A \rightarrow \boldsymbol{X}$ which is smooth of the minimal possible relative dimension $n=\operatorname{dim} H^{1}\left(\left.\mathbb{L}_{\boldsymbol{X}}\right|_{p}\right)$.

(c) An inclusion $\iota: B \hookrightarrow A$ of $B$ as a dg-subalgebra of $A$, so that $\boldsymbol{i}=$ Spec $\iota: U \rightarrow \boldsymbol{V}$ is a morphism of derived $\mathbb{K}$-schemes. On classical schemes, $i=t_{0}(\boldsymbol{i}): U=t_{0}(\boldsymbol{U}) \rightarrow V=t_{0}(\boldsymbol{V})$ is an isomorphism.

(d) $A \mathbb{K}$-point $\tilde{p} \in \operatorname{Spec} H^{0}(A)$ with $\varphi(\tilde{p})=p$, such that the 'standard form' cdgas $A, B$ have the minimal possible numbers of generators $\operatorname{dim} H^{j}\left(\left.\mathbb{L}_{\boldsymbol{U}}\right|_{\tilde{p}}\right)$, $\operatorname{dim} H^{j}\left(\left.\mathbb{L}_{\boldsymbol{V}}\right|_{\boldsymbol{i}(\tilde{p})}\right)$ in each degree $j=0,-1, \ldots, k, k-1$.

(e) An equivalence of relative (co)tangent complexes $\mathbb{L}_{\boldsymbol{U} / \boldsymbol{V}} \simeq \mathbb{T}_{\boldsymbol{U} / \boldsymbol{X}}[1-k]$. Hence $\mathbb{L}_{\boldsymbol{U} / \boldsymbol{V}}$ is a vector bundle of rank $n$ in degree $k-1$. 
(f) A k-shifted symplectic structure $\omega_{B}=\left(\omega_{B}^{0}, 0, \ldots\right)$ on $\boldsymbol{V}=\mathbf{S p e c} B$ which is in 'Darboux form' in the sense of [4, §5] and \$2.4 with $\varphi^{*}\left(\omega_{\boldsymbol{X}}\right) \sim \boldsymbol{i}^{*}\left(\omega_{B}\right)$ in $k$-shifted closed 2 -forms on $\boldsymbol{U}$.

For example, if $k=-2 d-1$ for $d=0,1, \ldots$ then the 'standard form' and 'Darboux form' conditions above mean the following. The degree 0 part $B^{0}$ of $B$ is a smooth $\mathbb{K}$-algebra of dimension $m_{0}$, and we are given $x_{1}^{0}, \ldots, x_{m_{0}}^{0} \in B^{0}$ such that $\left(x_{1}^{0}, \ldots, x_{m_{0}}^{0}\right)$ are étale coordinates on all of $V(0)=\operatorname{Spec} B^{0}$. As a graded commutative algebra, $B$ is freely generated over $B^{0}$ by variables

$$
\begin{array}{ll}
x_{1}^{-i}, \ldots, x_{m_{i}}^{-i} & \text { in degree }-i \text { for } i=1, \ldots, d, \\
y_{1}^{i-2 d-1}, \ldots, y_{m_{i}}^{i-2 d-1} & \text { in degree } i-2 d-1 \text { for } i=0,1, \ldots, d .
\end{array}
$$

We have $\omega_{B}^{0}=\sum_{i=0}^{d} \sum_{j=1}^{m_{i}} \mathrm{~d}_{d R} y_{j}^{i-2 d-1} \mathrm{~d}_{d R} x_{j}^{-i}$ in $\left(\Lambda^{2} \Omega_{B}^{1}\right)^{-2 d-1}$. The differential d on the cdga $B$ is $\mathrm{d} b=\{H, b\}$ for $b \in B$, where $\{\}:, B \times B \rightarrow B$ is the Poisson bracket defined using the inverse of $\omega_{B}^{0}$, and $H \in B^{-2 d}$ is a Hamiltonian function satisfying the classical master equation $\{H, H\}=0$. Also $B \subset A$, and $A$ is freely generated as a graded commutative algebra over $B$ by additional variables $w_{1}^{-2 d-2}, \ldots, w_{n}^{-2 d-2}$ in degree $-2 d-2$.

Theorem 1.1 says that given a $k$-shifted derived Artin stack $\left(\boldsymbol{X}, \omega_{\boldsymbol{X}}\right)$ for $k<0$, near each $p \in \boldsymbol{X}(\mathbb{K})$ we can find a smooth atlas $\boldsymbol{\varphi}: \boldsymbol{U} \rightarrow \boldsymbol{X}$ with $\boldsymbol{U}=\operatorname{Spec} A$ an affine derived scheme, such that $\left(\boldsymbol{U}, \boldsymbol{\varphi}^{*}\left(\omega_{\boldsymbol{X}}\right)\right)$ is in a standard 'Darboux form'. Although $\left(\boldsymbol{U}, \boldsymbol{\varphi}^{*}\left(\omega_{\boldsymbol{X}}\right)\right)$ is not $k$-shifted symplectic, as $\boldsymbol{\varphi}^{*}\left(\omega_{\boldsymbol{X}}\right)$ is not nondegenerate, we can build from $\left(\boldsymbol{U}, \boldsymbol{\varphi}^{*}\left(\omega_{\boldsymbol{X}}\right)\right)$ in a natural way a 'Darboux form' $k$-shifted symplectic derived scheme $\left(\boldsymbol{V}, \omega_{B}\right)$, which is equivalent to $\left(\boldsymbol{U}, \boldsymbol{\varphi}^{*}\left(\omega_{\boldsymbol{X}}\right)\right)$ except in degree $k-1$.

Theorem 1.2. Let $\left(\boldsymbol{X}, \omega_{\boldsymbol{X}}\right)$ be a-1-shifted symplectic derived Artin $\mathbb{K}$-stack in the sense of [30] over $\mathbb{K}$ algebraically closed of characteristic zero, and $X=$ $t_{0}(\boldsymbol{X})$ the corresponding classical Artin $\mathbb{K}$-stack. Then $X$ extends naturally to a d-critical stack $(X, s)$ in the sense of [15]. If $T$ is a $\mathbb{K}$-scheme and $t: T \rightarrow$ $X$ a smooth 1-morphism, this gives a d-critical structure $s(T, t)$ on $T$ making $(T, s(T, t))$ into an algebraic d-critical locus, in the sense of [15].

Theorem 1.2 implies that Artin moduli stacks $\mathcal{M}$ of (complexes of) coherent sheaves on a Calabi-Yau 3-fold $Y$ extend naturally to d-critical stacks $(\mathcal{M}, s)$.

Theorem 1.3. Let $(X, s)$ be an oriented d-critical stack over an algebraically closed field $\mathbb{K}$ with char $\mathbb{K} \neq 2$. Fix a theory of perverse sheaves or $\mathscr{D}$-modules over $\mathbb{K}$-schemes and Artin $\mathbb{K}$-stacks, for instance Laszlo and Olsson's l-adic perverse sheaves $[22[24]$. Then there is a natural perverse sheaf or $\mathscr{D}$-module $\check{P}_{X, s}^{\bullet}$ on $X$ with Verdier duality and monodromy isomorphisms

$$
\Sigma_{X, s}: \check{P}_{X, s}^{\bullet} \longrightarrow \mathbb{D}_{X}\left(\check{P}_{X, s}^{\bullet}\right), \quad \mathrm{T}_{X, s}: \check{P}_{X, s}^{\bullet} \longrightarrow \check{P}_{X, s}^{\bullet},
$$

such that if $T$ is a $\mathbb{K}$-scheme and $t: T \rightarrow X$ a 1-morphism smooth of relative dimension $n$, then $t^{*}\left(\check{P}_{X, s}^{\bullet}\right)[n], t^{*}\left(\Sigma_{X, s}\right)[n], t^{*}\left(\mathrm{~T}_{X, s}\right)[n]$ are isomorphic to the perverse sheaf or $\mathscr{D}$-module $P_{T, s(T, t)}^{\bullet}$ on the oriented algebraic d-critical locus 
$(T, s(T, t))$ defined in [3, §6], and its Verdier duality and monodromy isomorphisms $\Sigma_{T, s(T, t)}, \mathrm{T}_{T, s(T, t)}$. So in particular, if $(T, s(T, t))$ is locally modelled on a critical locus $\operatorname{Crit}\left(f: U \rightarrow \mathbb{A}^{1}\right)$ for $U$ a smooth $\mathbb{K}$-scheme, then $t^{*}\left(\check{P}_{X, s}^{\bullet}\right)[n]$ is locally modelled on the perverse sheaf or $\mathscr{D}$-module of vanishing cycles of $f$.

Theorem 1.4. Let $(X, s)$ be an oriented d-critical stack over $\mathbb{K}$ algebraically closed of characteristic zero, with $X$ of finite type and locally a global quotient. Then there exists a unique motive $M F_{X, s}$ in a certain ring $\overline{\mathcal{M}}_{X}^{\text {st, }, \hat{\mu}}$ of $\hat{\mu}$-equivariant motives on $X$, such that if $T$ is a finite type $\mathbb{K}$-scheme and $t: T \rightarrow X$ is smooth of relative dimension $n$, so that $(T, s(T, t))$ is an oriented algebraic d-critical locus over $\mathbb{K}$, then

$$
t^{*}\left(M F_{X, s}\right)=\mathbb{L}^{n / 2} \odot M F_{T, s(T, t)} \quad \text { in } \overline{\mathcal{M}}_{T}^{\mathrm{st}, \hat{\mu}},
$$

where $M F_{T, s(T, t)} \in \overline{\mathcal{M}}_{T}^{\mathrm{st}, \hat{\mu}}$ is as in [5, §5]. So in particular, if $(T, s(T, t))$ is locally modelled on $\operatorname{Crit}\left(f: U \rightarrow \mathbb{A}^{1}\right)$ for $U$ a smooth $\mathbb{K}$-scheme, then $\mathbb{L}^{-n / 2} \odot$ $t^{*}\left(M F_{X, s}\right)$ is locally modelled on the motivic vanishing cycle $M F_{U, f}^{\operatorname{mot}, \phi}$ of $f$.

We expect that Theorems 1.3 and 1.4 will have applications in categorified and motivic extensions of Donaldson-Thomas theory of Calabi-Yau 3-folds, as in Kontsevich and Soibelman [18,19.

Conventions and notation. Throughout $\mathbb{K}$ will be an algebraically closed field with char $\mathbb{K}=0$, except that we allow $\mathbb{K}$ algebraically closed with char $\mathbb{K} \neq$ 2 in 84 . Classical $\mathbb{K}$-schemes and Artin $\mathbb{K}$-stacks will be written $W, X, Y, Z, \ldots$, and derived $\mathbb{K}$-schemes and derived Artin $\mathbb{K}$-stacks in bold as $\boldsymbol{W}, \boldsymbol{X}, \boldsymbol{Y}, \boldsymbol{Z}, \ldots$.

Basic references for $\mathbb{K}$-schemes are Hartshorne [12, for Artin $\mathbb{K}$-stacks Laumon and Moret-Bailly [21, and for derived $\mathbb{K}$-schemes and derived Artin $\mathbb{K}$ stacks Toën and Vezzosi 32,33.

All (classical) $\mathbb{K}$-schemes and Artin $\mathbb{K}$-stacks $X$ are assumed locally of finite type, except in $\$ 5$ when we assume they are of finite type. All derived $\mathbb{K}$-schemes and derived $\mathbb{K}$-stacks $\boldsymbol{X}$ are assumed to be locally finitely presented. We write $\mathrm{Sch}_{\mathbb{K}}$ for the category of $\mathbb{K}$-schemes, Art $\mathbb{K}_{\mathbb{K}}$ for the 2-category of Artin $\mathbb{K}$-stacks, $\mathbf{d S c h}_{\mathbb{K}}$ for the $\infty$-category of derived $\mathbb{K}$-schemes, and $\mathbf{d A r t}_{\mathbb{K}}$ for the $\infty$-category of derived Artin $\mathbb{K}$-stacks, and $t_{0}: \mathbf{d S c h}_{\mathbb{K}} \rightarrow \mathrm{Sch}_{\mathbb{K}}, t_{0}: \mathbf{d A r t}_{\mathbb{K}} \rightarrow \operatorname{Art}_{\mathbb{K}}$ for the classical truncation functors. Other notation generally follows the prequels [3, 5, 15] to this paper.

Acknowledgements. We would like to thank Tom Bridgeland, Sven Meinhardt, Balázs Szendrői, and Bertrand Toën for helpful conversations, and a referee for careful proofreading and useful comments. This research was supported by EPSRC Programme Grant EP/I033343/1. The first author acknowledges the support of the European Commission under the Marie Curie Programme which awarded him an IEF grant. The contents of this article reflect the views of the authors and not the views of the European Commission. 


\section{Local models for atlases of shifted symplectic derived stacks}

Sections 2.1 and 2.2 give background on derived algebraic geometry [32,33 and Pantev-Toën-Vaquié-Vezzosi's shifted symplectic structures [30, and $\$ 2.3-\$ 2.4$ recall the main definitions of $4, \S 4-\S 5]$. Then $\$ 2.5-\$ 2.7$, the new material in this section, generalize 2.3 2.4 to derived Artin stacks.

\subsection{Derived algebraic geometry}

We work in the context of Toën and Vezzosi's derived algebraic geometry [32]33], and Pantev, Toën, Vaquié and Vezzosi's theory of $k$-shifted symplectic structures on derived schemes and stacks 30 . This is a complex subject, and we give only a brief sketch to fix notation. A longer explanation suited to our needs can be found in [4, §2-§3].

Fix an algebraically closed base field $\mathbb{K}$, of characteristic zero. Toën and Vezzosi define the $\infty$-category $\mathbf{d S t}_{\mathbb{K}}$ of derived $\mathbb{K}$-stacks (or $D^{-}$-stacks) 33 , Def. 2.2.2.14], [32, Def. 4.2]. All derived $\mathbb{K}$-stacks $\boldsymbol{X}$ in this paper are assumed to be locally finitely presented. There is a spectrum functor

Spec : $\{$ commutative differential graded $\mathbb{K}$-algebras, degrees $\leqslant 0\} \longrightarrow \mathbf{d S t}_{\mathbb{K}}$.

All cdgas in this paper will be in degrees $\leqslant 0$. A derived $\mathbb{K}$-stack $\boldsymbol{X}$ is called an affine derived $\mathbb{K}$-scheme if $\boldsymbol{X}$ is equivalent in $\mathbf{d S t}_{\mathbb{K}}$ to $\mathbf{S p e c} A$ for some cdga $A$ over $\mathbb{K}$. As in [32, §4.2], a derived $\mathbb{K}$-stack $\boldsymbol{X}$ is called a derived $\mathbb{K}$-scheme if it may be covered by Zariski open $\boldsymbol{Y} \subseteq \boldsymbol{X}$ with $\boldsymbol{Y}$ an affine derived $\mathbb{K}$-scheme. Write $\mathbf{d} \mathbf{S} \mathbf{c h}_{\mathbb{K}}$ for the full $\infty$-subcategory of derived $\mathbb{K}$-schemes in $\mathbf{d S t}_{\mathbb{K}}$.

We call a derived $\mathbb{K}$-stack $\boldsymbol{X}$ a derived Artin $\mathbb{K}$-stack if it is $m$-geometric for some $m$ [33, Def. 1.3.3.1] and the underlying classical stack is 1-truncated (that is, just a stack, not a higher stack). Any such $\boldsymbol{X}$ admits a smooth surjective morphism $\varphi: \boldsymbol{U} \rightarrow \boldsymbol{X}$, an atlas, with $\boldsymbol{U}$ a derived $\mathbb{K}$-scheme. Write $\mathbf{d A r t}_{\mathbb{K}}$ for the full $\infty$-subcategory of derived Artin $\mathbb{K}$-stacks in $\mathbf{d S t}_{\mathbb{K}}$. Then $\mathbf{d} \mathbf{S c h} \mathbf{K}_{\mathbb{K}} \subset$ $\mathbf{d A r t}_{\mathbb{K}} \subset \mathbf{d S t} \mathbf{t}_{\mathbb{K}}$.

Write $\mathrm{Sch}_{\mathbb{K}}$ for the category of $\mathbb{K}$-schemes $X$, and $\operatorname{Art}_{\mathbb{K}}$ for the 2-category of Artin $\mathbb{K}$-stacks $X$. By an abuse of notation we regard $S h_{\mathbb{K}}$ as a discrete 2subcategory of $\operatorname{Art}_{\mathbb{K}}$, so that $\operatorname{Sch}_{\mathbb{K}} \subset \operatorname{Art}_{\mathbb{K}}$. As in [33, Prop. 2.1.2.1], there is an inclusion functor $i:$ Art $_{\mathbb{K}} \rightarrow \mathbf{d A r t}_{\mathbb{K}}$ mapping $\mathbf{S c h}_{\mathbb{K}} \rightarrow \mathbf{d S c h}_{\mathbb{K}}$, and a classical truncation functor $t_{0}: \mathbf{d A r t}_{\mathbb{K}} \rightarrow \mathrm{Art}_{\mathbb{K}}$ mapping $\mathbf{d S c h}_{\mathbb{K}} \rightarrow \mathrm{Sch}_{\mathbb{K}}$.

A derived Artin $\mathbb{K}$-stack $\boldsymbol{X}$ has a cotangent complex $\mathbb{L}_{\boldsymbol{X}}$ of finite cohomological amplitude $[-m, 1]$ and a dual tangent complex $\mathbb{T}_{\boldsymbol{X}}[33, \S 1.4]$, 32, §4.2.4$\S 4.2 .5]$ in a stable $\infty$-category $L_{\mathrm{qcoh}}(\boldsymbol{X})$ defined in [32, §3.1.7, §4.2.4]. When $X$ is a classical scheme or stack, then the homotopy category of $L_{\mathrm{qcoh}}(\boldsymbol{X})$ is nothing but the triangulated category $D_{\mathrm{qcoh}}(X)$. These have the usual properties of (co)tangent complexes. For instance, if $\boldsymbol{f}: \boldsymbol{X} \rightarrow \boldsymbol{Y}$ is a morphism in $\mathbf{d A r t}_{\mathbb{K}}$ there is a distinguished triangle

$$
f^{*}\left(\mathbb{L}_{\boldsymbol{Y}}\right) \stackrel{\mathbb{L}_{\boldsymbol{f}}}{\longrightarrow} \mathbb{L}_{\boldsymbol{X}} \longrightarrow \mathbb{L}_{\boldsymbol{X} / \boldsymbol{Y}} \longrightarrow \boldsymbol{f}^{*}\left(\mathbb{L}_{\boldsymbol{Y}}\right)[1],
$$

where $\mathbb{L}_{\boldsymbol{X} / \boldsymbol{Y}}$ is the relative cotangent complex of $\boldsymbol{f}$. Here $\boldsymbol{f}$ is smooth of relative dimension $n$ if and only if $\mathbb{L}_{\boldsymbol{X} / \boldsymbol{Y}}$ is locally free of rank $n$, and $\boldsymbol{f}$ is étale if and only if $\mathbb{L}_{\boldsymbol{X} / \boldsymbol{Y}}=0$. 


\subsection{Shifted symplectic derived schemes and derived stacks}

Let $\boldsymbol{X}$ be a derived stack. Pantev, Toën, Vaquié and Vezzosi 30 defined $k$ shifted p-forms, $k$-shifted closed $p$-forms, and $k$-shifted symplectic structures on $\boldsymbol{X}$, for $k \in \mathbb{Z}$ and $p \geqslant 0$. One first defines these notions on derived affine schemes and then defines the general notions by smooth descent. Since our main theorems are statements about the local structure of derived stacks endowed with shifted symplectic forms, it suffices for us to describe the affine case. The basic idea is this:

(a) Define the exterior powers $\Lambda^{p} \mathbb{L}_{\boldsymbol{X}}$ in $L_{\mathrm{qcoh}}(\boldsymbol{X})$ for $p=0,1, \ldots$ Regard $\Lambda^{p} \mathbb{L}_{\boldsymbol{X}}$ as a complex, with differential d:

$$
\cdots \stackrel{\mathrm{d}}{\longrightarrow}\left(\Lambda^{p} \mathbb{L}_{\boldsymbol{X}}\right)^{k-1} \stackrel{\mathrm{d}}{\longrightarrow}\left(\Lambda^{p} \mathbb{L}_{\boldsymbol{X}}\right)^{k} \stackrel{\mathrm{d}}{\longrightarrow}\left(\Lambda^{p} \mathbb{L}_{\boldsymbol{X}}\right)^{k+1} \stackrel{\mathrm{d}}{\longrightarrow} \cdots .
$$

Then a $k$-shifted $p$-form, or $p$-form of degree $k$, is an element $\omega^{0}$ of $\left(\Lambda^{p} \mathbb{L}_{\boldsymbol{X}}\right)^{k}$ with $\mathrm{d} \omega^{0}=0$. Mostly we are interested in the cohomology class $\left[\omega^{0}\right] \in H^{k}\left(\Lambda^{p} \mathbb{L}_{\boldsymbol{X}}\right)$.

(b) There are de Rham differentials $\mathrm{d}_{d R}: \Lambda^{p} \mathbb{L}_{\boldsymbol{X}} \rightarrow \Lambda^{p+1} \mathbb{L}_{\boldsymbol{X}}$ with $\mathrm{d}_{d R} \circ \mathrm{d}_{d R}=$ $\mathrm{d} \circ \mathrm{d}_{d R}+\mathrm{d}_{d R} \circ \mathrm{d}=0$. Then a $k$-shifted closed $p$-form, or closed p-form of degree $k$, is a sequence $\omega=\left(\omega^{0}, \omega^{1}, \omega^{2}, \ldots\right)$ with $\omega^{i}$ in $\left(\Lambda^{p+i} \mathbb{L}_{\boldsymbol{X}}\right)^{k-i}$ for $i \geqslant 0$, satisfying $\mathrm{d} \omega^{0}=0$ and $\mathrm{d}_{d R} \omega^{i}+\mathrm{d} \omega^{i+1}=0$ for $i=0,1, \ldots$.

That is, $\omega=\left(\omega^{0}, \omega^{1}, \omega^{2}, \ldots\right)$ is a $k$-cycle in the negative cyclic complex

$$
\left(\left(\prod_{i=0}^{\infty}\left(\Lambda^{p+i} \mathbb{L}_{\boldsymbol{X}}\right)^{k-i}\right)_{k \in \mathbb{Z}}, \mathrm{d}+\mathrm{d}_{d R}\right) .
$$

Mostly we are interested in the cohomology class $[\omega]=\left[\omega^{0}, \omega^{1}, \ldots\right]$ in the cohomology of this complex. We will write $\omega \sim \omega^{\prime}$ if $\omega, \omega^{\prime}$ are $k$-shifted closed $p$-forms with the same cohomology class $[\omega]=\left[\omega^{\prime}\right]$. There is a map $\left(\omega^{0}, \omega^{1}, \omega^{2}, \ldots\right) \mapsto \omega^{0}$ from $k$-shifted closed $p$-forms to $k$-shifted $p$-forms.

(c) A $k$-shifted symplectic structure on $\boldsymbol{X}$ is a $k$-shifted closed 2 -form $\left(\omega^{0}, \ldots\right)$ on $\boldsymbol{X}$ whose induced morphism $\omega^{0} \cdot: \mathbb{T}_{\boldsymbol{X}} \rightarrow \mathbb{L}_{\boldsymbol{X}}[k]$ is an equivalence.

If a derived $\mathbb{K}$-scheme $\boldsymbol{X}$ has a 0 -shifted symplectic structure then $\boldsymbol{X}$ is a smooth $\mathbb{K}$-scheme $X$ with a classical symplectic structure. Pantev et al. [30] construct $k$-shifted symplectic structures on several classes of derived moduli stacks. If $Y$ is a Calabi-Yau $m$-fold and $\mathcal{M}$ a derived moduli stack of coherent sheaves or perfect complexes on $Y$, then $\mathcal{M}$ has a $(2-m)$-shifted symplectic structure. We are particularly interested in the case $m=3$, so $k=-1$.

\section{3 'Standard form' affine derived schemes}

The next definition summarizes [4, Ex. 2.8, Def. 2.9 \& Def. 2.13].

Definition 2.1. We will explain how to inductively construct a sequence of commutative differential graded algebras (cdgas) $A(0), A(1), \ldots, A(n)=A$ over $\mathbb{K}$ with $A(0)$ a smooth $\mathbb{K}$-algebra and $A(k)$ having underlying commutative graded algebra free over $A(0)$ on generators of degrees $-1, \ldots,-k$. We will call $A$ a standard form cdga. We will write $\boldsymbol{U}(i)=\mathbf{S p e c} A(i)$ for $i=0, \ldots, n$ and 
$\boldsymbol{U}=\boldsymbol{U}(n)=\operatorname{Spec} A$ for the corresponding affine derived $\mathbb{K}$-schemes, where $\boldsymbol{U}(0)=U(0)$ is a smooth classical $\mathbb{K}$-scheme, which contains $\operatorname{Spec} H^{0}(A)$ as a closed $\mathbb{K}$-subscheme.

Begin with a commutative algebra $A(0)$ smooth over $\mathbb{K}$. Choose a free $A(0)$ module $M^{-1}$ of finite rank together with a map $\pi^{-1}: M^{-1} \rightarrow A(0)$. Define a cdga $A(1)$ whose underlying commutative graded algebra is free over $A(0)$ with generators given by $M^{-1}$ in degree -1 and with differential d determined by the map $\pi^{-1}: M^{-1} \rightarrow A(0)$. By construction, we have $H^{0}(A(1))=A(0) / I$, where the ideal $I \subseteq A(0)$ is the image of the map $\pi^{-1}: M^{-1} \rightarrow A(0)$.

Note that $A(1)$ fits in a homotopy pushout diagram of cdgas

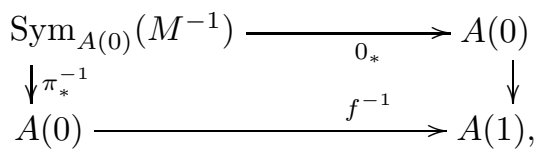

with morphisms $\pi_{*}^{-1}, 0_{*}$ induced by $\pi^{-1}, 0: M^{-1} \rightarrow A(0)$. Write $f^{-1}: A(0) \rightarrow$ $A(1)$ for the resulting map of algebras.

Next, choose a free $A(1)$-module $M^{-2}$ of finite rank together with a map $\pi^{-2}: M^{-2}[1] \rightarrow A(1)$. Define a cdga $A(2)$ whose underlying commutative graded algebra is free over $A(1)$ with generators given by $M^{-2}$ in degree -2 and with differential d determined by the map $\pi^{-2}: M^{-2}[1] \rightarrow A(1)$. Write $f^{-2}$ for the resulting map of algebras $A(1) \rightarrow A(2)$.

As the underlying commutative graded algebra of $A(1)$ was free over $A(0)$ on generators of degree -1 , the underlying commutative graded algebra of $A(2)$ is free over $A(0)$ on generators of degrees $-1,-2$. Since $A(2)$ is obtained from $A(1)$ by adding generators in degree -2 , we have $H^{0}(A(1)) \cong H^{0}(A(2)) \cong A(0) / I$.

Note that $A(2)$ fits in a homotopy pushout diagram of cdgas

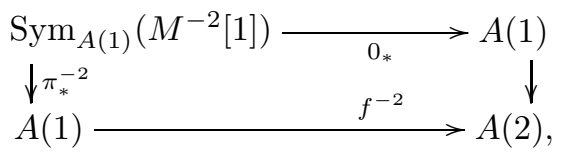

with morphisms $\pi_{*}^{-2}, 0_{*}$ induced by $\pi^{-2}, 0: M^{-2}[1] \rightarrow A(1)$.

Continuing in this manner inductively, we define a cdga $A(n)=A$ with $A^{0}=A(0)$ and $H^{0}(A)=A(0) / I$, whose underlying commutative graded algebra is free over $A(0)$ on generators of degrees $-1, \ldots,-n$. We call any cdga $A$ constructed in this way a standard form cdga.

If $A$ is of standard form, we will call a cdga $A^{\prime}$ a localization of $A$ if $A^{\prime}=$ $A \otimes_{A^{0}} A^{0}\left[f^{-1}\right]$ for $f \in A^{0}$, that is, $A^{\prime}$ is obtained by inverting $f$ in $A$. Then $A^{\prime}$ is also of standard form, with $A^{\prime 0} \cong A^{0}\left[f^{-1}\right]$. If $p \in \operatorname{Spec} H^{0}(A)$ with $f(p) \neq 0$, we call $A^{\prime}$ a localization of $A$ around $p$.

Let $A$ be a standard form cdga. We call $A$ minimal at $p \in \operatorname{Spec} H^{0}(A)$ if for all $k=1, \ldots, n$ the compositions

$$
H^{-k}\left(\mathbb{L}_{A(k) / A(k-1)}\right) \longrightarrow H^{1-k}\left(\mathbb{L}_{A(k-1)}\right) \longrightarrow H^{1-k}\left(\mathbb{L}_{A(k-1) / A(k-2)}\right)
$$

in the cotangent complexes restricted to $\operatorname{Spec} H^{0}(A)$ vanish at $p$. (For more on this point, see [4, Prop. 2.12].) 
Here are [4, Th.s $4.1 \& 4.2$. They say that any derived scheme $\boldsymbol{X}$ is locally modelled on Spec $A$ for a (minimal) standard form cdga $A$, and give us a way to compare two such local models $\boldsymbol{f}: \operatorname{Spec} A \hookrightarrow \boldsymbol{X}, \boldsymbol{g}: \operatorname{Spec} B \hookrightarrow \boldsymbol{X}$.

Theorem 2.2. Let $\boldsymbol{X}$ be a derived $\mathbb{K}$-scheme, and $x \in \boldsymbol{X}$. Then there exist a standard form cdga $A$ over $\mathbb{K}$ which is minimal at a point $p \in \operatorname{Spec} H^{0}(A)$, in the sense of Definition 2.1, and a morphism $\boldsymbol{f}: \boldsymbol{U}=\mathbf{S p e c} A \rightarrow \boldsymbol{X}$ in $\mathbf{d S c h}_{\mathbb{K}}$ which is a Zariski open inclusion with $\boldsymbol{f}(p)=x$.

Theorem 2.3. Let $\boldsymbol{X}$ be a derived $\mathbb{K}$-scheme, $A, B$ be standard form cdgas over $\mathbb{K}$, and $\boldsymbol{f}: \operatorname{Spec} A \rightarrow \boldsymbol{X}, \boldsymbol{g}:$ Spec $B \rightarrow \boldsymbol{X}$ be Zariski open inclusions in $\mathbf{d S c h}_{\mathbb{K}}$. Suppose $p \in \operatorname{Spec} H^{0}(A)$ and $q \in \operatorname{Spec} H^{0}(B)$ with $\boldsymbol{f}(p)=\boldsymbol{g}(q)$ in $\boldsymbol{X}$. Then there exist a standard form cdga $C$ over $\mathbb{K}$ which is minimal at $r$ in Spec $H^{0}(C)$ and morphisms of cdgas $\alpha: A \rightarrow C, \beta: B \rightarrow C$ which are Zariski open inclusions, such that $\mathbf{S p e c} \alpha: r \mapsto p, \operatorname{Spec} \beta: r \mapsto q$, and $\boldsymbol{f} \circ \mathbf{S p e c} \alpha \simeq \boldsymbol{g} \circ \mathbf{S p e c} \beta$ as morphisms $\mathbf{S p e c} C \rightarrow \boldsymbol{X}$ in $\mathbf{d S c h}_{\mathbb{K}}$.

If instead $\boldsymbol{f}, \boldsymbol{g}$ are étale rather than Zariski open inclusions, the same holds with $\alpha, \beta$ étale rather than Zariski open inclusions.

One important advantage of working with derived schemes $\boldsymbol{U}=\operatorname{Spec} A$ for $A$ a standard form cdga, is that the cotangent complex $\mathbb{L}_{U}$ and its exterior powers $\Lambda^{p} \mathbb{L}_{U}$ can be written simply and explicitly in terms of $A$. As in $[4, \S 2$, $\S 3.3]$ the differential-graded module of Kähler differentials $\Omega_{A}^{1}$ is a model for $\mathbb{L}_{U}$. If $U(0)=\operatorname{Spec} A^{0}$ admits global étale coordinates $\left(x_{1}^{0}, \ldots, x_{m_{0}}^{0}\right)$, then $\Omega_{A}^{1}$ is a finitely-generated free $A$-module, generated by $\mathrm{d}_{d R} x_{1}^{-i}, \ldots, \mathrm{d}_{d R} x_{m_{i}}^{-i}$ in degree $-i$ for $i=0, \ldots, n$, where $x_{1}^{-i}, \ldots, x_{m_{i}}^{-i}$ are $A(i-1)$-bases for the free finite rank $A(i-1)$-modules $M^{-i}$ for $i=1, \ldots, n$, in the notation of Definition 2.1.

Because of this, on $\boldsymbol{U}=\operatorname{Spec} A$, the $k$-shifted (closed) $p$-forms from [30] discussed in 2.2 can be written down explicitly in coordinates. Here is [4, Prop. 5.7]. Part (a) implies that for a $k$-shifted symplectic form $\omega=\left(\omega^{0}\right.$, $\omega^{1}, \omega^{2}, \ldots$ ) on a standard form $\boldsymbol{U}=\operatorname{Spec} A$, up to equivalence we may take $\omega^{1}=\omega^{2}=\cdots=0$, which simplifies calculations a lot. (Let us note here that the proof of [4, Prop. 5.7] uses the interpretation of shifted symplectic forms as representing classes in negative cyclic homology.)

Proposition 2.4. (a) Let $\omega=\left(\omega^{0}, \omega^{1}, \omega^{2}, \ldots\right)$ be a closed 2-form of degree $k<0$ on $\boldsymbol{U}=\operatorname{Spec} A$, for $A$ a standard form cdga over $\mathbb{K}$. Then there exist $\Phi \in A^{k+1}$ and $\phi \in\left(\Omega_{A}^{1}\right)^{k}$ such that $\mathrm{d} \Phi=0$ in $A^{k+2}$ and $\mathrm{d}_{d R} \Phi+\mathrm{d} \phi=0$ in $\left(\Omega_{A}^{1}\right)^{k+1}$ and $\omega \sim\left(\mathrm{d}_{d R} \phi, 0,0, \ldots\right)$.

(b) In the case $k=-1$ in (a) we have $\Phi \in A^{0}=A(0)$, so we can consider the restriction $\left.\Phi\right|_{U^{\text {red }}}$ of $\Phi$ to the reduced $\mathbb{K}$-subscheme $U^{\text {red }}$ of $U=t_{0}(\boldsymbol{U})=$ Spec $H^{0}(A)$. Then $\left.\Phi\right|_{U^{\mathrm{red}}}$ is locally constant on $U^{\mathrm{red}}$, and we may choose $(\Phi, \phi)$ in (a) such that $\left.\Phi\right|_{U^{\text {red }}}=0$.

(c) Suppose $(\Phi, \phi)$ and $\left(\Phi^{\prime}, \phi^{\prime}\right)$ are alternative choices in part (a) for fixed $\omega, k, \boldsymbol{U}, A$, where if $k=-1$ we suppose $\left.\Phi\right|_{U^{\mathrm{red}}}=0=\left.\Phi^{\prime}\right|_{U^{\mathrm{red}}}$ as in $(\mathbf{b})$. Then there exist $\Psi \in A^{k}$ and $\psi \in\left(\Omega_{A}^{1}\right)^{k-1}$ with $\Phi-\Phi^{\prime}=\mathrm{d} \Psi$ and $\phi-\phi^{\prime}=\mathrm{d}_{d R} \Psi+\mathrm{d} \psi$. 


\section{4 'Darboux form' shifted symplectic derived schemes}

The next definition summarizes [4, Ex.s 5.8-5.10].

Definition 2.5. Fix $d=0,1, \ldots$ We will explain how to define a class of explicit standard form cdgas $(A, \mathrm{~d})=A(n)$ for $n=2 d+1$ with a very simple, explicit $k$-shifted symplectic form $\omega=\left(\omega^{0}, 0,0, \ldots\right)$ on $\boldsymbol{U}=\operatorname{Spec} A$ for $k=-2 d-1$. We will say that $A, \omega$ are in Darboux form.

First choose a smooth $\mathbb{K}$-algebra $A(0)$ of dimension $m_{0}$. Localizing $A(0)$ if necessary, we may assume that there exist $x_{1}^{0}, \ldots, x_{m_{0}}^{0} \in A(0)$ such that $\mathrm{d}_{d R} x_{1}^{0}, \ldots, \mathrm{d}_{d R} x_{m_{0}}^{0}$ form a basis of $\Omega_{A(0)}^{1}$ over $A(0)$. Geometrically, $U(0)=$ Spec $A(0)$ is a smooth $\mathbb{K}$-scheme of dimension $m_{0}$, and $\left(x_{1}^{0}, \ldots, x_{m_{0}}^{0}\right): U(0) \rightarrow$ $\mathbb{A}^{m_{0}}$ are global étale coordinates on $U(0)$.

Next, choose $m_{1}, \ldots, m_{d} \in \mathbb{N}=\{0,1, \ldots\}$. Define $A$ as a commutative graded algebra to be the free algebra over $A(0)$ generated by variables

$$
\begin{array}{ll}
x_{1}^{-i}, \ldots, x_{m_{i}}^{-i} & \text { in degree }-i \text { for } i=1, \ldots, d, \text { and } \\
y_{1}^{i-2 d-1}, \ldots, y_{m_{i}}^{i-2 d-1} & \text { in degree } i-2 d-1 \text { for } i=0,1, \ldots, d .
\end{array}
$$

So the upper index $i$ in $x_{j}^{i}, y_{j}^{i}$ always indicates the degree. We will define the differential $\mathrm{d}$ in the cdga $(A, \mathrm{~d})$ later.

The spaces $\left(\Lambda^{p} \Omega_{A}^{1}\right)^{k}$ and the de Rham differential $\mathrm{d}_{d R}$ upon them depend only on the commutative graded algebra $A$, not on the (not yet defined) differ-

ential d. Note that $\Omega_{A}^{1}$ is the free $A$-module with basis $\mathrm{d}_{d R} x_{j}^{-i}, \mathrm{~d}_{d R} y_{j}^{i-2 d-1}$ for $i=0, \ldots, d$ and $j=1, \ldots, m_{i}$. Define

$$
\omega^{0}=\sum_{i=0}^{d} \sum_{j=1}^{m_{i}} \mathrm{~d}_{d R} y_{j}^{i-2 d-1} \mathrm{~d}_{d R} x_{j}^{-i} \quad \text { in }\left(\Lambda^{2} \Omega_{A}^{1}\right)^{-2 d-1} .
$$

Then $\mathrm{d}_{d R} \omega^{0}=0$ in $\left(\Lambda^{3} \Omega_{A}^{1}\right)^{-2 d-1}$.

Now choose $H$ in $A^{-2 d}$, which we will call the Hamiltonian, and which we require to satisfy the classical master equation

$$
\sum_{i=1}^{d} \sum_{j=1}^{m_{i}} \frac{\partial H}{\partial x_{j}^{-i}} \frac{\partial H}{\partial y_{j}^{i-2 d-1}}=0 \quad \text { in } A^{1-2 d}
$$

The classical master equation can be expressed invariantly as $\{H, H\}=0$, where $\{$,$\} is a certain shifted Poisson bracket. For more on this, consult [4, §5.7].$

Note that (2.4) is trivial when $d=0$, so that $k=-1$, as $A^{1}=0$. Define the differential d on $A$ by $\mathrm{d}=0$ on $A(0)$, and

$$
\mathrm{d} x_{j}^{-i}=\frac{\partial H}{\partial y_{j}^{i-2 d-1}}, \quad \mathrm{~d} y_{j}^{i-2 d-1}=\frac{\partial H}{\partial x_{j}^{-i}}, \quad \begin{aligned}
& i=0, \ldots, d, \\
& j=1, \ldots, m_{i} .
\end{aligned}
$$

Then $\mathrm{d} \circ \mathrm{d}=0$, and $(A, \mathrm{~d})$ is a standard form cdga $A=A(n)$ as in Definition 2.1 for $n=2 d+1$, defined using free modules $M^{-i}=\left\langle x_{1}^{-i}, \ldots, x_{m_{i}}^{-i}\right\rangle_{A(i-1)}$ for $i=1, \ldots, d$ and $M^{i-2 d-1}=\left\langle y_{1}^{i-2 d-1}, \ldots, y_{m_{i}}^{i-2 d-1}\right\rangle_{A(2 d-i)}$ for $i=0, \ldots, d$. 
Then $\omega=\left(\omega^{0}, 0,0, \ldots\right)$ is a $k$-shifted symplectic structure on $\boldsymbol{U}=\operatorname{Spec} A$ for $k=-2 d-1$. Define $\Phi \in A^{-2 d}$ and $\phi \in\left(\Omega_{A}^{1}\right)^{-2 d-1}$ by $\Phi=-\frac{1}{2 d+1} H$ and

$$
\phi=\frac{1}{2 d+1} \sum_{i=0}^{d} \sum_{j=1}^{m_{i}}\left[(2 d+1-i) y_{j}^{i-2 d-1} \mathrm{~d}_{d R} x_{j}^{-i}+i x_{j}^{-i} \mathrm{~d}_{d R} y_{j}^{i-2 d-1}\right] .
$$

Then $\mathrm{d} \Phi=0, \mathrm{~d}_{d R} \Phi+\mathrm{d} \phi=0$, and $\omega^{0}=\mathrm{d}_{d R} \phi$, as in Proposition 2.4(a). We say that $A, \omega$ are in Darboux form for $k=-2 d-1$.

In [4, Ex.s $5.9 \& 5.10]$ we give similar Darboux forms for $k=-4 d$ and $k=-4 d-2$ with $d=0,1,2, \ldots$ We will not give all the details. In brief, when $k=-4 d$, rather than (2.2), $A$ is freely generated over $A(0)$ by the variables

$$
\begin{array}{ll}
x_{1}^{-i}, \ldots, x_{m_{i}}^{-i} & \text { in degree }-i \text { for } i=1, \ldots, 2 d-1, \\
x_{1}^{-2 d}, \ldots, x_{m_{2 d}}^{-2 d}, y_{1}^{-2 d}, \ldots, y_{m_{2 d}}^{-2 d} & \text { in degree }-2 d, \text { and } \\
y_{1}^{i-4 d}, \ldots, y_{m_{i}}^{i-4 d} & \text { in degree } i-4 d \text { for } i=0,1, \ldots, 2 d-1,
\end{array}
$$

and $\omega^{0} \in\left(\Lambda^{2} \Omega_{A}^{1}\right)^{-4 d}$ with $\mathrm{d}_{d R} \omega^{0}=0$ is given by

$$
\omega^{0}=\sum_{i=0}^{2 d} \sum_{j=1}^{m_{i}} \mathrm{~d}_{d R} y_{j}^{i-4 d} \mathrm{~d}_{d R} x_{j}^{-i} \quad \text { in }\left(\Lambda^{2} \Omega_{A}^{1}\right)^{-4 d},
$$

and $\mathrm{d}$ on $A$ is defined as in (2.5) using $H \in A^{1-4 d}$ satisfying the analogue of (2.4). We then say that $A, \boldsymbol{U}=\mathbf{S p e c} A, \omega$ are in Darboux form for $k=-4 d$.

Similarly, when $k=-4 d-2, A$ is freely generated over $A(0)$ by the variables

$$
\begin{array}{ll}
x_{1}^{-i}, \ldots, x_{m_{i}}^{-i} & \text { in degree }-i \text { for } i=1, \ldots, 2 d, \\
z_{1}^{-2 d-1}, \ldots, z_{m_{2 d+1}}^{-2 d-1} & \text { in degree }-2 d-1, \text { and } \\
y_{1}^{i-4 d-2}, \ldots, y_{m_{i}}^{i-4 d-2} & \text { in degree } i-4 d-2 \text { for } i=0,1, \ldots, 2 d,
\end{array}
$$

and $\omega^{0} \in\left(\Lambda^{2} \Omega_{A}^{1}\right)^{-4 d-2}$ with $\mathrm{d}_{d R} \omega^{0}=0$ is given by

$$
\omega^{0}=\sum_{i=0}^{2 d} \sum_{j=1}^{m_{i}} \mathrm{~d}_{d R} y_{j}^{i-4 d-2} \mathrm{~d}_{d R} x_{j}^{-i}+\sum_{j=1}^{m_{2 d+1}} \mathrm{~d}_{d R} z_{j}^{-2 d-1} \mathrm{~d}_{d R} z_{j}^{-2 d-1},
$$

and $\mathrm{d}$ is defined as in (2.5) using $H \in A^{-4 d-1}$ satisfying

$$
\sum_{i=1}^{2 d} \sum_{j=1}^{m_{i}} \frac{\partial H}{\partial x_{j}^{-i}} \frac{\partial H}{\partial y_{j}^{i-4 d-2}}+\frac{1}{4} \sum_{j=1}^{m_{2 d+1}}\left(\frac{\partial H}{\partial z_{j}^{-2 d-1}}\right)^{2}=0 \quad \text { in } A^{-4 d} .
$$

We then say that $A, \omega$ are in strong Darboux form for $k=-4 d-2$. There is also a weak Darboux form [4, Ex. 5.12] in this case, which we will not discuss.

Here is [4, Th. 5.18], the main result of [4. We consider it to be a shifted symplectic analogue of Darboux' Theorem, as it shows that we can choose 'coordinate systems' on a $k$-shifted symplectic derived scheme $(\boldsymbol{X}, \omega)$ in which $\omega$ assumes a standard form.

Theorem 2.6. Let $\boldsymbol{X}$ be a derived $\mathbb{K}$-scheme with $k$-shifted symplectic form $\tilde{\omega}$ for $k<0$, and $x \in \boldsymbol{X}$. Then there exists a standard form cdga $A$ over $\mathbb{K}$ which is minimal at $p \in \operatorname{Spec} H^{0}(A)$, a k-shifted symplectic form $\omega$ on $\operatorname{Spec} A$, and a morphism $\boldsymbol{f}: \boldsymbol{U}=\operatorname{Spec} A \rightarrow \boldsymbol{X}$ with $\boldsymbol{f}(p)=x$ and $\boldsymbol{f}^{*}(\tilde{\omega}) \sim \omega$, such that: 
(i) If $k$ is odd or divisible by 4 , then $\boldsymbol{f}$ is a Zariski open inclusion, and $A, \omega$ are in Darboux form, as in Definition 2.5.

(ii) If $k \equiv 2 \bmod 4$, then $\boldsymbol{f}$ is étale, and $A, \omega$ are in strong Darboux form, as in Definition 2.5 .

Bouaziz and Grojnowski 2 also independently prove a similar theorem.

\section{5 'Standard form' atlases for derived stacks}

We first generalize Definition 2.1 and Theorems 2.2 2.3 to derived Artin stacks:

Definition 2.7. Let $\boldsymbol{X}$ be a derived Artin $\mathbb{K}$-stack, and $p$ a point of $\boldsymbol{X}$. By this we mean a morphism $p: \operatorname{Spec} \mathbb{K} \rightarrow \boldsymbol{X}$; we may also call $p$ a $\mathbb{K}$-point of $\boldsymbol{X}$. A standard form open neighbourhood $(A, \boldsymbol{\varphi}, \tilde{p})$ of $p$, in the smooth topology, means a standard form cdga $A$ over $\mathbb{K}$ in the sense of Definition 2.1, so that $\boldsymbol{U}=\operatorname{Spec} A$ is an affine derived $\mathbb{K}$-scheme, and a morphism $\boldsymbol{\varphi}: \boldsymbol{U} \rightarrow \boldsymbol{X}$ which is smooth of some relative dimension $n \geqslant 0$, and a $\mathbb{K}$-point $\tilde{p}$ in $\boldsymbol{U}$ with $p=\boldsymbol{\varphi}(\tilde{p})$, that is, there is an equivalence of morphisms $p \simeq \varphi \circ \tilde{p}: \operatorname{Spec} \mathbb{K} \rightarrow \boldsymbol{X}$. If we do not specify $p, \tilde{p}$, we just call $(A, \varphi)$ a standard form open neighbourhood in $\boldsymbol{X}$.

For such $\boldsymbol{X}, p,(A, \boldsymbol{\varphi}, \tilde{p}), n$, as for (2.1) we have the standard fibre sequence

$$
\varphi^{*}\left(\mathbb{L}_{X}\right) \stackrel{\mathbb{L}_{\varphi}}{\longrightarrow} \mathbb{L}_{U} \longrightarrow \mathbb{L}_{U / X} \longrightarrow \varphi^{*}\left(\mathbb{L}_{X}\right)[1]
$$

where $\mathbb{L}_{\boldsymbol{U} / \boldsymbol{X}}$ is locally free of rank $n$. Restricting (2.7) to $\tilde{p}$ and taking cohomology, we have the following:

(a) There are isomorphisms $H^{i}\left(\left.\mathbb{L}_{\boldsymbol{X}}\right|_{p}\right) \cong H^{i}\left(\left.\mathbb{L}_{\boldsymbol{U}}\right|_{\tilde{p}}\right)$ for $i<0$.

(b) Since $\boldsymbol{U}$ is not stacky, $H^{1}\left(\left.\mathbb{L}_{\boldsymbol{U}}\right|_{\tilde{p}}\right)=0$ and so there is an exact sequence of $\mathbb{K}$-vector spaces

$$
0 \longrightarrow H^{0}\left(\left.\mathbb{L}_{\boldsymbol{X}}\right|_{p}\right) \rightarrow H^{0}\left(\left.\mathbb{L}_{\boldsymbol{U}}\right|_{\tilde{p}}\right) \rightarrow H^{0}\left(\left.\mathbb{L}_{\boldsymbol{U} / \boldsymbol{X}}\right|_{\tilde{p}}\right) \rightarrow H^{1}\left(\left.\mathbb{L}_{\boldsymbol{X}}\right|_{p}\right) \rightarrow 0
$$

where $H^{0}\left(\left.\mathbb{L}_{\boldsymbol{U} / \boldsymbol{X}}\right|_{\tilde{p}}\right) \cong \mathbb{K}^{n}$. Therefore $n \geqslant \operatorname{dim} H^{1}\left(\left.\mathbb{L}_{\boldsymbol{X}}\right|_{p}\right)$.

Note that $H^{1}\left(\left.\mathbb{L}_{\boldsymbol{X}}\right|_{p}\right) \cong \mathfrak{I s o}_{\boldsymbol{X}}(p)^{*}$, where $\mathfrak{I s o}_{\boldsymbol{X}}(p)$ is the Lie algebra of the isotropy group $\operatorname{Iso}_{\boldsymbol{X}}(p)$ of $\boldsymbol{X}$ at $p$, which is an algebraic $\mathbb{K}$-group.

In particular, the minimal possible relative dimension $n=\operatorname{rank}\left(\mathbb{L}_{\boldsymbol{U} / \boldsymbol{X}}\right)$ of a neighbourhood $\varphi: \boldsymbol{U} \rightarrow \boldsymbol{X}$ of $p$ is $n=\operatorname{dim} H^{1}\left(\left.\mathbb{L}_{\boldsymbol{X}}\right|_{p}\right)$.

(c) If $\boldsymbol{\varphi}$ is smooth of minimal relative dimension $n=\operatorname{dim} H^{1}\left(\left.\mathbb{L}_{\boldsymbol{X}}\right|_{p}\right)$, then

$$
H^{0}\left(\left.\mathbb{L}_{\boldsymbol{X}}\right|_{p}\right) \cong H^{0}\left(\left.\mathbb{L}_{\boldsymbol{U}}\right|_{\tilde{p}}\right) \text { and } H^{0}\left(\left.\mathbb{L}_{\boldsymbol{U} / \boldsymbol{X}}\right|_{\tilde{p}}\right) \cong H^{1}\left(\left.\mathbb{L}_{\boldsymbol{X}}\right|_{p}\right)
$$

We call a standard form open neighbourhood $(A, \varphi, \tilde{p})$ minimal at $p$ if $A$ is minimal at $\tilde{p}$ in the sense of Definition 2.1 and $n=\operatorname{dim} H^{1}\left(\left.\mathbb{L}_{\boldsymbol{X}}\right|_{p}\right)$. Then parts (a),(c) imply that $A(0)$ is smooth of dimension $m_{0}=\operatorname{dim} H^{0}\left(\left.\mathbb{L}_{\boldsymbol{X}}\right|_{p}\right)$, and $A$ has $m_{i}=\operatorname{dim} H^{-i}\left(\left.\mathbb{L}_{\boldsymbol{X}}\right|_{p}\right)$ generators in degree $-i$ for $i=1,2, \ldots$. 
Theorem 2.8. Let $\boldsymbol{X}$ be a derived Artin $\mathbb{K}$-stack, and $p$ a point of $\boldsymbol{X}$. Then there exists a minimal standard form open neighbourhood $(A, \boldsymbol{\varphi}, \tilde{p})$ of $p$, in the sense of Definition 2.7.

Proof. Since $\boldsymbol{X}$ has a smooth atlas, for any $p \in \boldsymbol{X}$ there exists an affine neighbourhood $\hat{\boldsymbol{\varphi}}: \hat{\boldsymbol{U}} \rightarrow \boldsymbol{X}$ of $p$, where $\hat{\boldsymbol{U}}$ is an affine derived $\mathbb{K}$-scheme, $\hat{p} \in \hat{\boldsymbol{U}}$ with $\hat{\boldsymbol{\varphi}}(\hat{p})=p$, and $\hat{\boldsymbol{\varphi}}$ is smooth of some relative dimension $\hat{n}$, with $\hat{n} \geqslant \operatorname{dim} H^{1}\left(\left.\mathbb{L}_{\boldsymbol{X}}\right|_{p}\right)$ by Definition $2.7(\mathrm{~b})$. Let $r=\hat{n}-\operatorname{dim} H^{1}\left(\left.\mathbb{L}_{\boldsymbol{X}}\right|_{p}\right)$, so that $r$ is the dimension of the kernel of $H^{0}\left(\left.\mathbb{L}_{\hat{\boldsymbol{U}} / \boldsymbol{X}}\right|_{\hat{p}}\right) \rightarrow H^{1}\left(\left.\mathbb{L}_{\boldsymbol{X}}\right|_{p}\right) \rightarrow 0$. We shall use this kernel to cut down $\hat{\boldsymbol{\varphi}}: \hat{\boldsymbol{U}} \rightarrow \boldsymbol{X}$ to the minimal dimension $n=\operatorname{dim} H^{1}\left(\left.\mathbb{L}_{\boldsymbol{X}}\right|_{p}\right)$.

Localizing $\hat{\boldsymbol{U}}$ around $\hat{p}$, by Theorem 2.2 we may take $\hat{\boldsymbol{U}}=\operatorname{Spec} \hat{A}$, where $\hat{A}$ is a standard form cdga minimal at $\hat{p} \in \hat{\boldsymbol{U}}$. Then the natural map $H^{0}\left(\left.\mathbb{L}_{\hat{U}(0)}\right|_{\hat{p}}\right) \rightarrow$ $H^{0}\left(\left.\mathbb{L}_{\hat{\boldsymbol{U}}}\right|_{\hat{p}}\right)$ is an isomorphism. Since $H^{0}\left(\left.\mathbb{L}_{\hat{\boldsymbol{U}}}\right|_{\hat{p}}\right) \rightarrow H^{0}\left(\left.\mathbb{L}_{\hat{\boldsymbol{U}} / \boldsymbol{X}}\right|_{\hat{p}}\right) \rightarrow H^{1}\left(\left.\mathbb{L}_{\boldsymbol{X}}\right|_{p}\right)$ is exact, we may choose (after localization) functions $x_{1}, \ldots, x_{r}$ on $\hat{U}(0)$ vanishing at $\hat{p}$ so that $\mathrm{d}_{d R} x_{1}, \ldots, \mathrm{d}_{d R} x_{r}$ at $\hat{p}$ map to a basis of the kernel of $H^{0}\left(\left.\mathbb{L}_{\hat{\boldsymbol{U}} / \boldsymbol{X}}\right|_{\hat{p}}\right) \rightarrow$ $H^{1}\left(\left.\mathbb{L}_{\boldsymbol{X}}\right|_{p}\right)$ under the composition $H^{0}\left(\left.\mathbb{L}_{\hat{U}(0)}\right|_{\hat{p}}\right) \rightarrow H^{0}\left(\left.\mathbb{L}_{\hat{\boldsymbol{U}}}\right|_{\hat{p}}\right) \rightarrow H^{0}\left(\left.\mathbb{L}_{\hat{\boldsymbol{U}} / \boldsymbol{X}}\right|_{\hat{p}}\right)$.

The functions $x_{1}, \ldots, x_{r}$ define a map $g: \hat{U}(0) \rightarrow \mathbb{A}^{r}$ and hence a map $\boldsymbol{f}: \hat{\boldsymbol{U}} \rightarrow \mathbb{A}^{r}$ with $\boldsymbol{f}(\hat{p})=0$. We let $\boldsymbol{U}$ denote the (homotopy) fibre $\boldsymbol{f}^{-1}(0)$, so that we have the following diagram in which the square is a pullback:

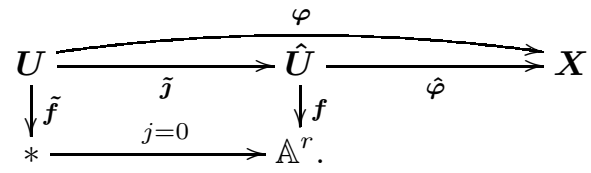

Let $\tilde{p}$ be the preimage of $\hat{p}$ in $\boldsymbol{U}$. We will show that after localizing $\boldsymbol{U}$ around $\tilde{p}$, the composition $\boldsymbol{\varphi}=\hat{\boldsymbol{\varphi}} \circ \tilde{\boldsymbol{j}}: \boldsymbol{U} \rightarrow \boldsymbol{X}$ is smooth of relative dimension $n=\hat{n}-r=\operatorname{dim} H^{1}\left(\left.\mathbb{L}_{\boldsymbol{X}}\right|_{p}\right)$. Consider the fibre sequence $\mathbb{L}_{\boldsymbol{U} / \hat{\boldsymbol{U}}}[-1] \rightarrow$ $\tilde{\boldsymbol{\jmath}}^{*}\left(\mathbb{L}_{\hat{\boldsymbol{U}} / \boldsymbol{X}}\right) \rightarrow \mathbb{L}_{\boldsymbol{U} / \boldsymbol{X}}$. We claim $\mathbb{L}_{\boldsymbol{U} / \hat{\boldsymbol{U}}}[-1]$ is free of rank $r$ and that the map $\mathbb{L}_{\boldsymbol{U} / \hat{\boldsymbol{U}}}[-1] \rightarrow \tilde{\boldsymbol{J}}^{*}\left(\mathbb{L}_{\hat{\boldsymbol{U} / \boldsymbol{X}}}\right)$ is injective at $\tilde{p}$ and hence, by Nakayama's Lemma, in a neighbourhood of $\tilde{p}$. Localizing $\boldsymbol{U}$ around $\tilde{p}$, it will follow immediately that $\mathbb{L}_{\boldsymbol{U} / \boldsymbol{X}}$ is locally free of rank $n=\hat{n}-r$. Thus $\boldsymbol{\varphi}: \boldsymbol{U} \rightarrow \boldsymbol{X}$ is the desired neighbourhood of $p$ of minimal relative dimension.

To sustain the claim, note that since the cotangent complex of $*=\operatorname{Spec} \mathbb{K}$ is zero, we have an equivalence $\mathbb{L}_{j}[-1] \simeq j^{*}\left(\Omega_{\mathbb{A}^{r}}^{1}\right)$. Thus $\mathbb{L}_{j}[-1]$ is free of rank $r$ and hence so is $\tilde{\boldsymbol{f}}^{*}\left(\mathbb{L}_{j}\right)[-1] \simeq \mathbb{L}_{\boldsymbol{U} / \hat{\boldsymbol{U}}}[-1]$. Furthermore, the map in question

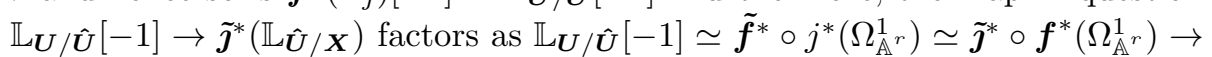
$\tilde{\boldsymbol{J}}^{*}\left(\mathbb{L}_{\hat{\boldsymbol{U}}}\right) \rightarrow \tilde{\boldsymbol{J}}^{*}\left(\mathbb{L}_{\hat{\boldsymbol{U}} / \boldsymbol{X}}\right)$. But $\boldsymbol{f}$ was constructed precisely so that the composition $f^{*}\left(\Omega_{\mathbb{A}^{r}}^{1}\right) \rightarrow \mathbb{L}_{\hat{\boldsymbol{U}}} \rightarrow \mathbb{L}_{\hat{\boldsymbol{U}} / \boldsymbol{X}}$ should be injective at $\hat{p}$. Thus, we may choose an affine neighbourhood $\boldsymbol{\varphi}: \boldsymbol{U} \rightarrow \boldsymbol{X}, \tilde{p}$ of $p$ which is smooth of the minimal relative dimension $n=\operatorname{dim} H^{1}\left(\left.\mathbb{L}_{\boldsymbol{X}}\right|_{p}\right)$. Applying Theorem 2.2 to $\boldsymbol{U}$ at $\tilde{p}$, we may take $\boldsymbol{U}=\operatorname{Spec} A$, where $A$ is a standard form cdga minimal at $\tilde{p} \in \boldsymbol{U}$.

Theorem 2.9. Let $\boldsymbol{X}$ be a derived Artin $\mathbb{K}$-stack and $(A, \boldsymbol{\varphi}),(B, \boldsymbol{\psi})$ standard form open neighbourhoods in $\boldsymbol{X}$, and write $\boldsymbol{U}=\operatorname{Spec} A, \boldsymbol{V}=\operatorname{Spec} B$. Then for each $p \in \boldsymbol{U} \times_{\boldsymbol{X}} \boldsymbol{V}$ there exist a standard form cdga $C$ over $\mathbb{K}$ minimal at 
$q \in \boldsymbol{W}=\operatorname{Spec} C$, an étale morphism $\boldsymbol{i}: \boldsymbol{W} \rightarrow \boldsymbol{U} \times_{\boldsymbol{X}} \boldsymbol{V}$ with $\boldsymbol{i}(q)=p$, and cdga morphisms $\alpha: A \rightarrow C, \beta: B \rightarrow C$ with $\boldsymbol{\pi}_{\boldsymbol{U}} \circ \boldsymbol{i} \simeq \operatorname{Spec} \alpha: \boldsymbol{W} \rightarrow \boldsymbol{U}$ and $\boldsymbol{\pi}_{\boldsymbol{V}} \circ \boldsymbol{i} \simeq \operatorname{Spec} \beta: \boldsymbol{W} \rightarrow \boldsymbol{V}$.

Proof. Since $\boldsymbol{U}, \boldsymbol{V}$ are derived $\mathbb{K}$-schemes, $\boldsymbol{U} \times_{\boldsymbol{X}} \boldsymbol{V}$ is a derived algebraic $\mathbb{K}$ space, and étale locally equivalent to a derived $\mathbb{K}$-scheme. Thus given $p \in$ $\boldsymbol{U} \times{ }_{\boldsymbol{X}} \boldsymbol{V}$ we may choose an affine derived $\mathbb{K}$-scheme $\hat{\boldsymbol{W}}$, a point $\hat{q} \in \hat{\boldsymbol{W}}$ and an étale map $\hat{\boldsymbol{\imath}}: \hat{\boldsymbol{W}} \rightarrow \boldsymbol{U} \times_{\boldsymbol{X}} \boldsymbol{V}$ with $\hat{\boldsymbol{\imath}}(\hat{q})=p$.

Write $\hat{W}=t_{0}(\hat{\boldsymbol{W}}), U(0)=\operatorname{Spec} A^{0}$ and $V(0)=\operatorname{Spec} B^{0}$ for the classical schemes. The compositions $\hat{W} \hookrightarrow \hat{\boldsymbol{W}} \stackrel{\hat{\imath}}{\longrightarrow} \boldsymbol{U} \times_{\boldsymbol{X}} \boldsymbol{V} \stackrel{\boldsymbol{\pi}_{U}}{\longrightarrow} \boldsymbol{U} \hookrightarrow U(0)$ and $\hat{W} \hookrightarrow$ $\hat{\boldsymbol{W}} \stackrel{\hat{\imath}}{\longrightarrow} \boldsymbol{U} \times_{\boldsymbol{X}} \boldsymbol{V} \stackrel{\boldsymbol{\pi}_{\boldsymbol{V}}}{\longrightarrow} \boldsymbol{V} \hookrightarrow V(0)$ give maps $\hat{W} \rightarrow U(0), \hat{W} \rightarrow V(0)$. Choose a map $\hat{W} \rightarrow \mathbb{A}^{N}$ such that the product map $\hat{W} \rightarrow U(0) \times V(0) \times \mathbb{A}^{N}$ is a locally closed embedding. Localizing $\hat{\boldsymbol{W}}, \hat{W}$ at $\hat{q}$ if necessary, we can choose a locally closed $\mathbb{K}$-subscheme $W(0)$ of $U(0) \times V(0) \times \mathbb{A}^{N}$ containing the image of $\hat{W}$ as a closed $\mathbb{K}$-subscheme, such that $W(0)$ is smooth of dimension $\operatorname{dim} T_{\hat{q}} \hat{W}$. For instance, $W(0)$ can be obtained as an intersection of an appropriate regular sequence of hypersurfaces.

Following the proof of Theorem 2.2 in [4, §4.1], we can construct a standard form cdga $C$ minimal at $q \in \boldsymbol{W}=\operatorname{Spec} C$ with $\operatorname{Spec} C^{0}=W(0)$ and an equivalence $\boldsymbol{j}: \boldsymbol{W} \rightarrow \hat{\boldsymbol{W}}$ with $\boldsymbol{j}(q)=\hat{q}$. Setting $\boldsymbol{i}=\hat{\boldsymbol{\imath}} \circ \boldsymbol{j}$, we now have morphisms of derived schemes $\boldsymbol{\pi}_{\boldsymbol{U}} \circ \boldsymbol{i}: \boldsymbol{W} \rightarrow \boldsymbol{U}, \boldsymbol{\pi}_{\boldsymbol{V}} \circ \boldsymbol{i}: \boldsymbol{W} \rightarrow \boldsymbol{V}$ whose classical truncations $\pi_{U} \circ i: W \rightarrow U, \pi_{V} \circ i: W \rightarrow V$ extend to morphisms of the ambient smooth schemes $W(0)=\operatorname{Spec} C^{0} \rightarrow U(0)=\operatorname{Spec} A^{0}, W(0)=$ Spec $C^{0} \rightarrow V(0)=$ Spec $B^{0}$. As $A, B$ are freely generated in negative degrees, it follows that we may write $\boldsymbol{\pi}_{\boldsymbol{U}} \circ \boldsymbol{i} \simeq \mathbf{S p e c} \alpha$ and $\boldsymbol{\pi}_{\boldsymbol{V}} \circ \boldsymbol{i} \simeq \mathbf{S p e c} \beta$ for morphisms of cdgas $\alpha: A \rightarrow C, \beta: B \rightarrow C$. This completes the proof.

\section{6 'Darboux form' atlases for shifted symplectic stacks}

Here is the main result of this section, a stack analogue of Theorem 2.6 Note that (a)(i)-(v) are modelled closely on the first part of Definition 2.5, and equations (2.9)-(2.13) are analogues of or identical to (2.2) $-(2.6)$.

Theorem 2.10. (a) Let $\left(\boldsymbol{X}, \omega_{\boldsymbol{X}}\right)$ be a $k$-shifted symplectic derived Artin $\mathbb{K}$ stack, where $k=-2 d-1$ for $d=0,1,2, \ldots$, and $p \in \boldsymbol{X}$. Then we can construct a minimal standard form open neighbourhood $(A, \boldsymbol{\varphi}: \boldsymbol{U} \rightarrow \boldsymbol{X}, \tilde{p})$ of $p$ in the sense of Definition 2.7, and a k-shifted closed 2-form $\omega=\left(\omega^{0}, 0, \ldots\right)$ on $\boldsymbol{U}=$ Spec $A$ for $\omega^{0} \in\left(\Lambda^{2} \Omega_{A}^{1}\right)^{k}$, such that $\boldsymbol{\varphi}^{*}\left(\omega_{\boldsymbol{X}}\right) \sim \omega$ in $k$-shifted closed 2 -forms on $\boldsymbol{U}=\operatorname{Spec} A$. Furthermore, $A, \omega$ are in a standard 'Darboux form', a modified version of Definition 2.5 as follows:

(i) The degree 0 part $A^{0}$ of $A$ is a smooth $\mathbb{K}$-algebra of dimension $m_{0}$, and we are given $x_{1}^{0}, \ldots, x_{m_{0}}^{0} \in A^{0}$ such that $\mathrm{d}_{d R} x_{1}^{0}, \ldots, \mathrm{d}_{d R} x_{m_{0}}^{0}$ form a basis of $\Omega_{A^{0}}^{1}$ over $A^{0}$. 
(ii) As a graded commutative algebra, $A$ is freely generated over $A^{0}$ by variables

$$
\begin{array}{ll}
x_{1}^{-i}, \ldots, x_{m_{i}}^{-i} & \text { in degree }-i \text { for } i=1, \ldots, d, \\
y_{1}^{i-2 d-1}, \ldots, y_{m_{i}}^{i-2 d-1} & \text { in degree } i-2 d-1 \text { for } i=0,1, \ldots, d, \\
w_{1}^{-2 d-2}, \ldots, w_{n}^{-2 d-2} & \text { in degree }-2 d-2,
\end{array}
$$

for $m_{0}, \ldots, m_{d} \geqslant 0$ with $m_{0}$ as in (i), and $n=\operatorname{dim} H^{1}\left(\left.\mathbb{L}_{\boldsymbol{X}}\right|_{p}\right)$ the relative dimension of $\boldsymbol{\varphi}$. The upper index $i$ in $w_{j}^{i}, x_{j}^{i}, y_{j}^{i}$ is the degree. Then

$$
\omega^{0}=\sum_{i=0}^{d} \sum_{j=1}^{m_{i}} \mathrm{~d}_{d R} y_{j}^{i-2 d-1} \mathrm{~d}_{d R} x_{j}^{-i} \quad \text { in }\left(\Lambda^{2} \Omega_{A}^{1}\right)^{-2 d-1} .
$$

(iii) We are given $H$ in $A^{-2 d}$, called the Hamiltonian, which satisfies the classical master equation

$$
\sum_{i=1}^{d} \sum_{j=1}^{m_{i}} \frac{\partial H}{\partial x_{j}^{-i}} \frac{\partial H}{\partial y_{j}^{i-2 d-1}}=0 \quad \text { in } A^{1-2 d}
$$

The differential $\mathrm{d}$ on $A$ satisfies $\mathrm{d}=0$ on $A^{0}$, and

$$
\mathrm{d} x_{j}^{-i}=\frac{\partial H}{\partial y_{j}^{i-2 d-1}}, \quad \mathrm{~d} y_{j}^{i-2 d-1}=\frac{\partial H}{\partial x_{j}^{-i}}, \begin{aligned}
& i=0, \ldots, d, \\
& j=1, \ldots, m_{i} .
\end{aligned}
$$

Note that (2.12) does not specify $\mathrm{d} w_{j}^{-2 d-2}$ for $j=1, \ldots, n$, and so does not completely determine $\mathrm{d}$ on $A$.

(iv) Define $\Phi \in A^{-2 d}$ and $\phi \in\left(\Omega_{A}^{1}\right)^{-2 d-1}$ by $\Phi=-\frac{1}{2 d+1} H$ and

$$
\phi=\frac{1}{2 d+1} \sum_{i=0}^{d} \sum_{j=1}^{m_{i}}\left[(2 d+1-i) y_{j}^{i-2 d-1} \mathrm{~d}_{d R} x_{j}^{-i}+i x_{j}^{-i} \mathrm{~d}_{d R} y_{j}^{i-2 d-1}\right] .
$$

Then $\mathrm{d} \Phi=0, \mathrm{~d}_{d R} \Phi+\mathrm{d} \phi=0$, and $\omega^{0}=\mathrm{d}_{d R} \phi$.

(v) Minimality of $(A, \boldsymbol{\varphi}, \tilde{p})$ means that $\left.\mathrm{d} w_{j}^{-2 d-2}\right|_{\tilde{p}}=0$ for $j=1, \ldots, n$ and

$$
\left.\mathrm{d} x_{j}^{-i}\right|_{\tilde{p}}=\left.\frac{\partial H}{\partial y_{j}^{i-2 d-1}}\right|_{\tilde{p}}=0=\left.\mathrm{d} y_{j}^{i-2 d-1}\right|_{\tilde{p}}=\left.\frac{\partial H}{\partial x_{j}^{-i}}\right|_{\tilde{p}} \begin{aligned}
& i=0, \ldots, d, \\
& j=1, \ldots, m_{i} .
\end{aligned}
$$

(b) In part (a), let $B$ be the graded subalgebra of $A$ generated by $A^{0}$ and the variables $x_{j}^{i}, y_{j}^{i}$ in (ii) for all $i, j$, with inclusion $\iota: B \hookrightarrow A$. Then $B$ is closed under $\mathrm{d}$, and so is a dg-subalgebra of $A$. For degree reasons $H, \Phi$ above cannot depend on the $w_{j}^{-2 d-2}$, so $H, \Phi \in B$. Also the data $\omega, \omega^{0}, \phi$ in $\Omega_{A}^{1}, \Lambda^{2} \Omega_{A}^{1}$ above are the images under $\iota$ of $\omega_{B}, \omega_{B}^{0}, \phi_{B}$ in $\Omega_{B}^{1}, \Lambda^{2} \Omega_{B}^{1}$. Then $\omega_{B}$ is a $k$-shifted symplectic structure on $\boldsymbol{V}=\operatorname{Spec} B$, and $B, \omega_{B}$ is in Darboux form as in Definition 2.5, and $B$ is minimal at $\tilde{p}$ as in Definition 2.1.

Geometrically, we have a diagram of morphisms in $\mathbf{d A r t}_{\mathbb{K}}$ :

$$
\boldsymbol{V}=\operatorname{Spec} B \longleftarrow{ }_{i=\operatorname{Spec} \iota} U=\operatorname{Spec} A \longrightarrow \boldsymbol{\varphi},
$$

where $\left(\boldsymbol{X}, \omega_{\boldsymbol{X}}\right),\left(\boldsymbol{V}, \omega_{B}\right)$ are $k$-shifted symplectic, with $\boldsymbol{\varphi}^{*}\left(\omega_{\boldsymbol{X}}\right) \sim \boldsymbol{i}^{*}\left(\omega_{B}\right)$ in $k$ shifted closed 2-forms on $\boldsymbol{U}$. We can think of $\boldsymbol{\varphi}: \boldsymbol{U} \rightarrow \boldsymbol{X}$ as a 'submersion', 
and $\boldsymbol{i}: \boldsymbol{U} \hookrightarrow \boldsymbol{V}$ as an embedding of $\boldsymbol{U}$ as a derived subscheme of $\boldsymbol{V}$. On classical schemes, $i=t_{0}(\boldsymbol{i}): U=t_{0}(\boldsymbol{U}) \rightarrow V=t_{0}(\boldsymbol{V})$ is an isomorphism. There is a natural equivalence of relative (co)tangent complexes

$$
\mathbb{L}_{\boldsymbol{U} / \boldsymbol{V}} \simeq \mathbb{T}_{\boldsymbol{U} / \boldsymbol{X}}[1-k]
$$

(c) The obvious analogues of $(\mathbf{a}),(\mathbf{b})$ also hold if $\left(\boldsymbol{X}, \omega_{\boldsymbol{X}}\right)$ is a $k$-shifted symplectic derived Artin $\mathbb{K}$-stack for $k<0$ with $k \equiv 0 \bmod 4$ or $k \equiv 2 \bmod 4$. In each case, the algebra $A$ is the corresponding algebra from Definition 2.5] modified by adding generators $w_{1}^{k-1}, \ldots, w_{n}^{k-1}$ in degree $k-1$.

Proof. For $(\mathrm{a})$, let $\left(\boldsymbol{X}, \omega_{\boldsymbol{X}}\right)$ be a $k$-shifted symplectic derived Artin $\mathbb{K}$-stack with $k=-2 d-1$ for $d \geqslant 0$, and $p \in \boldsymbol{X}$. By Theorem 2.8 we may choose a minimal standard form open neighbourhood $(A, \varphi, \tilde{p})$ of $p$, which we may localize further during the proof. Then by Definition 2.7 $\varphi$ is smooth of relative dimension $n=\operatorname{dim} H^{1}\left(\left.\mathbb{L}_{\boldsymbol{X}}\right|_{p}\right)$, and $A(0)$ is smooth of dimension $m_{0}=\operatorname{dim} H^{0}\left(\left.\mathbb{L}_{\boldsymbol{X}}\right|_{p}\right)$, and $A$ has $m_{i}=\operatorname{dim} H^{-i}\left(\left.\mathbb{L}_{\boldsymbol{X}}\right|_{p}\right)$ generators in degree $-i$ for $i=1,2, \ldots$

Since $\left(\boldsymbol{X}, \omega_{\boldsymbol{X}}\right)$ is $k$-shifted symplectic for $k=-2 d-1$ we have $H^{-i}\left(\left.\mathbb{L}_{\boldsymbol{X}}\right|_{p}\right) \cong$ $H^{k+i}\left(\left.\mathbb{L}_{\boldsymbol{X}}\right|_{p}\right)^{*}$, so $\operatorname{dim} H^{-i}\left(\left.\mathbb{L}_{\boldsymbol{X}}\right|_{p}\right)=\operatorname{dim} H^{k+i}\left(\left.\mathbb{L}_{\boldsymbol{X}}\right|_{p}\right)$. Thus, $A$ is freely generated over $A^{0}$ by $m_{i}$ generators in degree $-i$ for $i=1, \ldots, d$, and $m_{i}$ generators in degree $i-2 d-1$ for $i=0,1, \ldots, d$, and $n$ generators in degree $-2 d-2$, which is the same number of variables as in (2.9).

The pullback $\varphi^{*}\left(\omega_{\boldsymbol{X}}\right)$ is a $k$-shifted closed 2 -form on $\boldsymbol{U}=\operatorname{Spec} A$, so Proposition 2.4 gives $\omega^{0} \in\left(\Omega_{A}^{2}\right)^{k}$ with $\mathrm{d} \omega^{0}=\mathrm{d}_{d R} \omega^{0}=0$ and $\boldsymbol{\varphi}^{*}\left(\omega_{\boldsymbol{X}}\right) \sim\left(\omega^{0}, 0,0, \ldots\right)$. Consider the morphism $\omega^{0} \cdot: \mathbb{T}_{A} \rightarrow \Omega_{A}^{1}[k]$ given by contraction with $\omega^{0}$, and its restriction to $\tilde{p}$ on cohomology, which gives morphisms

$$
H^{i}\left(\left.\omega^{0} \cdot\right|_{\tilde{p}}\right): H^{i}\left(\left.\mathbb{T}_{A}\right|_{\tilde{p}}\right) \cong H^{-i}\left(\left.\Omega_{A}^{1}\right|_{\tilde{p}}\right)^{*} \longrightarrow H^{k+i}\left(\left.\Omega_{A}^{1}\right|_{\tilde{p}}\right) .
$$

On cohomology $\omega^{0}$. factorizes as $\mathbb{T}_{A} \rightarrow \varphi^{*}\left(\mathbb{T}_{\boldsymbol{X}}\right) \rightarrow \varphi^{*}\left(\mathbb{L}_{\boldsymbol{X}}\right)[k] \rightarrow \Omega_{A}^{1}[k]$. Here $\boldsymbol{\varphi}^{*}\left(\mathbb{T}_{\boldsymbol{X}}\right) \rightarrow \boldsymbol{\varphi}^{*}\left(\mathbb{L}_{\boldsymbol{X}}\right)[k]$ is the pullback of $\omega_{\boldsymbol{X}} \cdot: \mathbb{T}_{\boldsymbol{X}} \rightarrow \mathbb{L}_{\boldsymbol{X}}[k]$, which is an equivalence as $\omega_{\boldsymbol{X}}$ is nondegenerate. Also $\boldsymbol{\varphi}^{*}\left(\mathbb{L}_{\boldsymbol{X}}\right)[k] \rightarrow \Omega_{A}^{1}[k]$ is $\mathbb{L}_{\boldsymbol{\varphi}}[k]$ as in (2.7), and so as in Definition 2.7, on cohomology $H^{i}$ at $\tilde{p}$ is an isomorphism for $i \leqslant-k$, and zero for $i=1-k$. The map $\mathbb{T}_{A} \rightarrow \varphi^{*}\left(\mathbb{T}_{\boldsymbol{X}}\right)$ is the dual of $\mathbb{L}_{\boldsymbol{\varphi}}$, and so on cohomology $H^{i}$ at $\tilde{p}$ is an isomorphism for $i \geqslant 0$, and zero for $i=-1$. Combining these, (2.15) is an isomorphism for $0 \leqslant i \leqslant-k$ and zero otherwise.

We can now prove (a)(i)-(iv) by following the proof of the $k$ odd case of Theorem 2.6] in [4, §5.6]. Localizing $A$ at $\tilde{p}$ if necessary, this chooses étale coordinates $x_{1}^{0}, \ldots, x_{m_{0}}^{0}$ on $U^{0}=\operatorname{Spec} A^{0}$, and generators $x_{1}^{-i}, \ldots, x_{m_{i}}^{-i}$ in degree $-i$ for $i=1, \ldots, d$ and $y_{1}^{i-2 d-1}, \ldots, y_{m_{i}}^{i-2 d-1}$ in degree $i-2 d-1$ for $i=0,1, \ldots, d$ for $A$, such that $\omega^{0}$ is given by (2.10), and also constructs $H, \Phi, \phi$ satisfying (2.11)(2.13). The proof in [4, §5.6] does not choose the generators $w_{1}^{-2 d-2}, \ldots, w_{n}^{-2 d-2}$ for $A$ in degree $-2 d-2$, but as these are not required to satisfy any conditions, they can be chosen arbitrarily. Note that $\omega^{0}, H, \Phi, \phi$ do not involve $w_{1}^{-2 d-2}, \ldots, w_{n}^{-2 d-2}$ for degree reasons. Part (a)(v) follows from Definition 2.7 and (2.12). This completes (a).

The first parts of (b) are immediate, comparing (a) with Definition 2.5. To construct the equivalence (2.14), consider the following diagram, in which the 
rows are the standard fibre sequences and the vertical arrow is induced by an inverse of $\varphi^{*}\left(\omega_{\boldsymbol{X}}\right)$ :

$$
\begin{gathered}
\mathbb{L}_{\boldsymbol{U} / \boldsymbol{X}}[-1] \longrightarrow \varphi^{*}\left(\mathbb{L}_{\boldsymbol{X}}\right) \longrightarrow \mathbb{L}_{\boldsymbol{U}} \\
\downarrow \simeq \\
\mathbb{T}_{\boldsymbol{U}}[-k] \longrightarrow \boldsymbol{\varphi}^{*}\left(\mathbb{T}_{\boldsymbol{X}}\right)[-k] \longrightarrow \mathbb{T}_{\boldsymbol{U} / \boldsymbol{X}}[1-k] .
\end{gathered}
$$

Since $\mathbb{L}_{\boldsymbol{U} / \boldsymbol{X}}$ and $\mathbb{T}_{\boldsymbol{U} / \boldsymbol{X}}$ can be assumed to be free, we have

$$
\begin{aligned}
& \operatorname{Ext}^{-1}\left(\mathbb{L}_{\boldsymbol{U} / \boldsymbol{X}}[-1], \mathbb{T}_{\boldsymbol{U} / \boldsymbol{X}}[1-k]\right) \cong \operatorname{Ext}^{1-k}\left(\mathbb{L}_{\boldsymbol{U} / \boldsymbol{X}}, \mathbb{T}_{\boldsymbol{U} / \boldsymbol{X}}\right)=0, \\
& \operatorname{Hom}\left(\mathbb{L}_{\boldsymbol{U} / \boldsymbol{X}}[-1], \mathbb{T}_{\boldsymbol{U} / \boldsymbol{X}}[1-k]\right) \cong \operatorname{Ext}^{-k+2}\left(\mathbb{L}_{\boldsymbol{U} / \boldsymbol{X}}, \mathbb{T}_{\boldsymbol{U} / \boldsymbol{X}}\right)=0 .
\end{aligned}
$$

Applying $\mathbb{R} \mathcal{H o m}\left(\mathbb{L}_{\boldsymbol{U} / \boldsymbol{X}}[-1],-\right)$ to the bottom row of (2.16) and taking cohomology, we find that $\operatorname{Hom}\left(\mathbb{L}_{\boldsymbol{U} / \boldsymbol{X}}[-1], \mathbb{T}_{\boldsymbol{U}}[-k]\right) \cong \operatorname{Hom}\left(\mathbb{L}_{\boldsymbol{U} / \boldsymbol{X}}[-1], \boldsymbol{\varphi}^{*}\left(\mathbb{T}_{\boldsymbol{X}}\right)[-k]\right)$. Thus (2.16) can be filled in to a commutative diagram

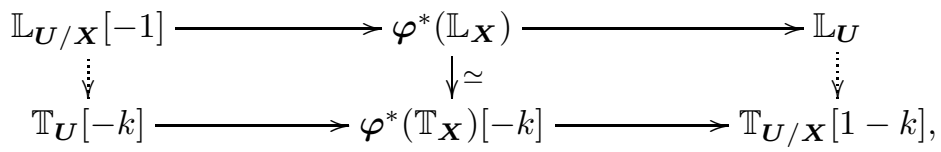

and such a filling is unique up to homotopy.

Restricting (2.17) to $\tilde{p}$ and taking cohomology gives a commutative diagram:

$$
\begin{aligned}
& H^{k-1}\left(\left.\mathbb{L}_{\boldsymbol{X}}\right|_{p}\right) \longrightarrow H^{k-1}\left(\left.\mathbb{L}_{\boldsymbol{U}}\right|_{\tilde{p}}\right) \\
& \downarrow \cong \\
& H^{k-1}\left(\left.\mathbb{T}_{\boldsymbol{X}}\right|_{p}[-k]\right) \longrightarrow H^{k-1}\left(\left.\mathbb{T}_{\boldsymbol{U} / \boldsymbol{X}}\right|_{\tilde{p}}[1-k]\right) .
\end{aligned}
$$

Since the morphism

$$
H^{-1}\left(\left.\mathbb{T}_{\boldsymbol{X}}\right|_{p}\right) \cong H^{k-1}\left(\left.\mathbb{T}_{\boldsymbol{X}}\right|_{p}[1-k]\right) \rightarrow H^{k-1}\left(\left.\mathbb{T}_{\boldsymbol{U} / \boldsymbol{X}}\right|_{\tilde{p}}[-k]\right) \cong H^{0}\left(\left.\mathbb{T}_{\boldsymbol{U} / \boldsymbol{X}}\right|_{\tilde{p}}\right)
$$

is dual to $H^{0}\left(\left.\mathbb{L}_{\boldsymbol{U} / \boldsymbol{X}}\right|_{\tilde{p}}\right) \rightarrow H^{1}\left(\left.\mathbb{L}_{\boldsymbol{X}}\right|_{p}\right)$, which is an isomorphism by (2.8), we see from (2.18) that $H^{k-1}\left(\left.\mathbb{L}_{\boldsymbol{U}}\right|_{\tilde{p}}\right) \rightarrow H^{k-1}\left(\left.\mathbb{T}_{\boldsymbol{U} / \boldsymbol{X}}\right|_{\tilde{p}}[1-k]\right)$ is also an isomorphism.

Next, consider the fibre sequence $\iota^{*}\left(\mathbb{L}_{\boldsymbol{V}}\right) \rightarrow \mathbb{L}_{\boldsymbol{U}} \rightarrow \mathbb{L}_{\boldsymbol{U} / \boldsymbol{V}}$. Note that $\mathbb{L}_{\boldsymbol{U} / \boldsymbol{V}}[k-1]$ is free of rank $\operatorname{dim} H^{k-1}\left(\left.\mathbb{L}_{\boldsymbol{X}}\right|_{p}\right)=\operatorname{dim} H^{1}\left(\left.\mathbb{L}_{\boldsymbol{X}}\right|_{p}\right)=n$ and that the natural map $H^{k-1}\left(\left.\mathbb{L}_{\boldsymbol{U}}\right|_{\tilde{p}}\right) \rightarrow H^{k-1}\left(\left.\mathbb{L}_{\boldsymbol{U} / \boldsymbol{V}}\right|_{\tilde{p}}\right)$ is an isomorphism by the minimality of the inductive construction of $\boldsymbol{U}=\operatorname{Spec} A$ in Definition 2.1.

Since $\iota^{*}\left(\mathbb{L}_{\boldsymbol{V}}\right)$ has amplitude in $[k, 0]$ and $\mathbb{T}_{\boldsymbol{U} / \boldsymbol{X}}$ is locally free, the composition $\iota^{*}\left(\mathbb{L}_{\boldsymbol{V}}\right) \rightarrow \mathbb{L}_{\boldsymbol{U}} \rightarrow \mathbb{T}_{\boldsymbol{U} / \boldsymbol{X}}[1-k]$ is homotopic to zero, and we can therefore choose a factorization $\mathbb{L}_{\boldsymbol{U}} \rightarrow \mathbb{L}_{\boldsymbol{U} / \boldsymbol{V}} \rightarrow \mathbb{T}_{\boldsymbol{U} / \boldsymbol{X}}[1-k]$ of $\mathbb{L}_{\boldsymbol{U}} \rightarrow \mathbb{T}_{\boldsymbol{U} / \boldsymbol{X}}[1-k]$. Restricting this factorization to $\tilde{p}$ and taking cohomology, we see that the induced map $H^{k-1}\left(\left.\mathbb{L}_{\boldsymbol{U} / \boldsymbol{V}}\right|_{\tilde{p}}\right) \rightarrow H^{k-1}\left(\left.\mathbb{T}_{\boldsymbol{U} / \boldsymbol{X}}\right|_{\tilde{p}}[1-k]\right)$ is an isomorphism. By Nakayama's Lemma, the map $\mathbb{L}_{\boldsymbol{U} / \boldsymbol{V}} \rightarrow \mathbb{T}_{\boldsymbol{U} / \boldsymbol{X}}[1-k]$ is an equivalence in a neighbourhood of $\tilde{p}$. So localizing $\boldsymbol{U}, \boldsymbol{V}$ if necessary, equation (2.14) holds, proving part (b).

For (c), we follow the same method, using the 'Darboux form' in [4, Ex. 5.9] for $k \equiv 0 \bmod 4$, and the 'strong Darboux form' in [4, Ex. 5.10] for $k \equiv 2$ 
$\bmod 4$. As in the proof of [4, Th. 5.18(iii)], in the case $k \equiv 2 \bmod 4$, as well as modifying $A$ by localizing at $\tilde{p}$ (i.e. restricting to a Zariski open neighbourhood of $\tilde{p}$ in $\boldsymbol{U}=\operatorname{Spec} A$ ), we also need to modify $A$ by adjoining square roots of some nonzero functions in $A^{0}$ (i.e. taking a finite étale cover of $\boldsymbol{U}=\operatorname{Spec} A$ ). As the result is still a minimal standard form open neighbourhood $(A, \varphi, \tilde{p})$ of $p$, this does not affect the statement of the theorem.

In the case $k=-1$, as in [4, Ex. 5.15] the classical $\mathbb{K}$-schemes $U \cong V$ in Theorem 2.10(a),(b) are isomorphic to $\operatorname{Crit}\left(H: U(0) \rightarrow \mathbb{A}^{1}\right)$. Also $\boldsymbol{\varphi}: \boldsymbol{T} \rightarrow \boldsymbol{X}$ smooth implies $\varphi=t_{0}(\boldsymbol{\varphi}): T=t_{0}(\boldsymbol{T}) \rightarrow X=t_{0}(\boldsymbol{X})$ is smooth. So changing notation from $U(0), H, \tilde{p}$ to $U, f, u$, using $H^{i}\left(\left.\mathbb{L}_{X}\right|_{p}\right) \cong H^{i}\left(\left.\mathbb{L}_{\boldsymbol{X}}\right|_{p}\right)$ for $X=t_{0}(\boldsymbol{X})$ and $i=0,1$, and applying Proposition 2.4(b) to get $\left.f\right|_{T^{\mathrm{red}}}=0$, we deduce:

Corollary 2.11. Let $\left(\boldsymbol{X}, \omega_{\boldsymbol{X}}\right)$ be a-1-shifted symplectic derived Artin $\mathbb{K}$-stack, and $X=t_{0}(\boldsymbol{X})$ the corresponding classical Artin $\mathbb{K}$-stack. Then for each $p \in X$ there exist a smooth $\mathbb{K}$-scheme $U$ with dimension $\operatorname{dim} H^{0}\left(\left.\mathbb{L}_{X}\right|_{p}\right)$, a point $t \in U$, a regular function $f: U \rightarrow \mathbb{A}^{1}$ with $\left.\mathrm{d}_{d R} f\right|_{t}=0$, so that $T:=\operatorname{Crit}(f) \subseteq U$ is a closed $\mathbb{K}$-subscheme with $t \in T$, and a morphism $\varphi: T \rightarrow X$ which is smooth of relative dimension $\operatorname{dim} H^{1}\left(\left.\mathbb{L}_{X}\right|_{p}\right)$, with $\varphi(t)=p$. We may take $\left.f\right|_{T^{\mathrm{red}}}=0$.

Here the derived critical locus $\operatorname{Crit}\left(f: U \rightarrow \mathbb{A}^{1}\right)$, as a-1-shifted symplectic derived scheme, agrees with $\left(\boldsymbol{V}, \omega_{B}\right)$ in Theorem 2.10 , and $\varphi: T \rightarrow X$ corresponds to $t_{0}(\boldsymbol{\varphi}) \circ t_{0}(\boldsymbol{i})^{-1}$ in Theorem 2.10 ,

Thus, the underlying classical stack $X$ of a -1-shifted symplectic derived stack $\left(\boldsymbol{X}, \omega_{\boldsymbol{X}}\right)$ admits an atlas consisting of critical loci of regular functions on smooth schemes.

Now let $Y$ be a Calabi-Yau 3 -fold over $\mathbb{K}$, and $\mathcal{M}$ a classical moduli stack of coherent sheaves $F$ on $Y$, or complexes $F^{\bullet}$ in $D^{b} \operatorname{coh}(Y)$ with $\operatorname{Ext}^{<0}\left(F^{\bullet}, F^{\bullet}\right)=$ 0 . Then $\mathcal{M}=t_{0}(\mathcal{M})$, for $\mathcal{M}$ the corresponding derived moduli stack. The (open) condition $\operatorname{Ext}^{<0}\left(F^{\bullet}, F^{\bullet}\right)=0$ is needed to make $\mathcal{M} 1$-truncated (that is, a derived Artin stack, in our terminology), and so make $\mathcal{M}=t_{0}(\mathcal{M})$ an ordinary, and not higher, stack. Pantev et al. [30, §2.1] prove $\mathcal{M}$ has a -1 -shifted symplectic structure $\omega_{\mathcal{M}}$. Applying Corollary 2.11] and using $H^{i}\left(\left.\mathbb{L}_{\mathcal{M}}\right|_{[F]}\right) \cong \operatorname{Ext}^{1-i}(F, F)^{*}$ yields a new result on classical 3-Calabi-Yau moduli stacks, the statement of which involves no derived geometry:

Corollary 2.12. Suppose $Y$ is a Calabi-Yau 3-fold over $\mathbb{K}$, and $\mathcal{M}$ a classical moduli $\mathbb{K}$-stack of coherent sheaves $F$, or more generally of complexes $F^{\bullet}$ in $D^{b} \operatorname{coh}(Y)$ with $\operatorname{Ext}^{<0}\left(F^{\bullet}, F^{\bullet}\right)=0$. Then for each $[F] \in \mathcal{M}$, there exist a

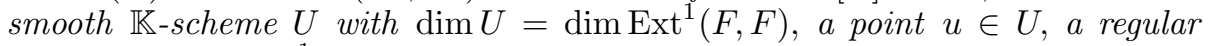
function $f: U \rightarrow \mathbb{A}^{1}$ with $\left.\mathrm{d}_{d R} f\right|_{u}=0$, and a morphism $\varphi: \operatorname{Crit}(f) \rightarrow \mathcal{M}$ which is smooth of relative dimension $\operatorname{dim} \operatorname{Hom}(F, F)$, with $\varphi(u)=[F]$.

This is an analogue of [4, Cor. 5.19]. When $\mathbb{K}=\mathbb{C}$, a related result for coherent sheaves only, with $U$ a complex manifold and $f$ a holomorphic function, was proved by Joyce and Song [16, Th. 5.5] using gauge theory and transcendental complex methods. 


\subsection{Comparing 'Darboux form' atlases on overlaps}

Let $\left(\boldsymbol{X}, \omega_{\boldsymbol{X}}\right)$ be a $k$-shifted symplectic derived Artin $\mathbb{K}$-stack for $k<0$. Theorem 2.10 gives a minimal standard form open neighbourhood $(A, \varphi, \tilde{p})$ of each $p$ in $\boldsymbol{X}$ with $\varphi^{*}\left(\omega_{\boldsymbol{X}}\right) \sim \omega$, where the $k$-shifted closed 2 -form $\omega=\left(\omega^{0}, 0, \ldots\right)$ on $\boldsymbol{U}=\operatorname{Spec} A$ is in a standard 'Darboux form', and $\Phi \in A^{k+1}, \phi \in\left(\Omega_{A}^{1}\right)^{k}$ with $\mathrm{d} \Phi=0, \mathrm{~d}_{d R} \Phi+\mathrm{d} \phi=0, \mathrm{~d}_{d R} \phi=\omega^{0}$, satisfying $\left.\Phi\right|_{U^{\mathrm{red}}}=0$ if $k=-1$, as in Proposition 2.4(a),(b). We think of $A, \boldsymbol{\varphi}, \omega, \Phi, \phi$ as like coordinates on $\boldsymbol{X}$ near $p$ in the smooth topology, which write $\boldsymbol{X}, \omega_{\boldsymbol{X}}$ in a nice way.

It is often important in geometric problems to compare different choices of coordinates on the overlap of their domains. So suppose $A, \boldsymbol{U}, \boldsymbol{\varphi}, \omega, \Phi, \phi$ and $A^{\prime}, \boldsymbol{U}^{\prime}, \varphi^{\prime}, \omega^{\prime}, \Phi^{\prime}, \phi^{\prime}$ are two choices as above, and $q \in \boldsymbol{U} \times \boldsymbol{\varphi}, \boldsymbol{X}, \boldsymbol{\varphi}^{\prime} \boldsymbol{U}^{\prime}$. We would like to compare the presentations $A, \boldsymbol{U}, \boldsymbol{\varphi}, \omega, \Phi, \phi$ and $A^{\prime}, \boldsymbol{U}^{\prime}, \varphi^{\prime}, \omega^{\prime}, \Phi^{\prime}, \phi^{\prime}$ for $\boldsymbol{X}$ near $q$. Here is a method for doing this, following [4, §5.8] in the scheme case:

(i) Apply Theorem 2.9 to $(A, \varphi),\left(A^{\prime}, \varphi^{\prime}\right), q$. This gives a standard form cdga $B$ minimal at $r \in \boldsymbol{V}=\operatorname{Spec} B$, an etale map $\boldsymbol{i}: \boldsymbol{V} \hookrightarrow \boldsymbol{U} \times{ }_{\boldsymbol{X}} \boldsymbol{U}^{\prime}$ with $\boldsymbol{i}(r)=q$, and morphisms of cdgas $\alpha: A \rightarrow B, \alpha^{\prime}: A^{\prime} \rightarrow B$ with $\boldsymbol{\pi}_{\boldsymbol{U}} \circ \boldsymbol{i} \simeq$ $\operatorname{Spec} \alpha: \boldsymbol{V} \rightarrow \boldsymbol{U}$ and $\boldsymbol{\pi}_{\boldsymbol{U}^{\prime}} \circ \boldsymbol{i} \simeq \operatorname{Spec} \alpha^{\prime}: \boldsymbol{V} \rightarrow \boldsymbol{U}^{\prime}$.

(ii) The pullbacks $\alpha_{*}(\omega)=\left(\alpha_{*}\left(\omega^{0}\right), 0, \ldots\right), \alpha_{*}^{\prime}\left(\omega^{\prime}\right)=\left(\alpha_{*}^{\prime}\left(\omega^{\prime 0}\right), 0, \ldots\right)$ are $k$ shifted closed 2-forms on $\boldsymbol{V}=\operatorname{Spec} B$, which are equivalent as

$$
\begin{aligned}
\alpha_{*}(\omega) & \sim(\operatorname{Spec} \alpha)^{*} \circ \varphi^{*}\left(\omega_{\boldsymbol{X}}\right) \sim \boldsymbol{i}^{*} \circ \boldsymbol{\pi}_{\boldsymbol{U}}^{*} \circ \varphi^{*}\left(\omega_{\boldsymbol{X}}\right) \\
& \sim \boldsymbol{i}^{*} \circ \boldsymbol{\pi}_{\boldsymbol{U}^{\prime}}^{*} \circ \boldsymbol{\varphi}^{\prime *}\left(\omega_{\boldsymbol{X}}\right) \sim\left(\operatorname{Spec} \alpha^{\prime}\right)^{*} \circ \boldsymbol{\varphi}^{\prime *}\left(\omega_{\boldsymbol{X}}\right) \sim \alpha_{*}^{\prime}\left(\omega^{\prime}\right) .
\end{aligned}
$$

Since $B$ is minimal at $r, \alpha_{*}(\omega), \alpha_{*}^{\prime}\left(\omega^{\prime}\right)$ satisfy nondegeneracy properties near $r$. Also $\mathrm{d} \alpha(\Phi)=0, \mathrm{~d}_{d R} \alpha(\Phi)+\mathrm{d} \alpha_{*}(\phi)=0, \mathrm{~d} \alpha^{\prime}\left(\Phi^{\prime}\right)=0, \mathrm{~d}_{d R} \alpha_{*}(\phi)=$ $\alpha_{*}\left(\omega^{0}\right), \mathrm{d}_{d R} \alpha^{\prime}\left(\Phi^{\prime}\right)+\mathrm{d} \alpha_{*}^{\prime}\left(\phi^{\prime}\right)=0, \mathrm{~d}_{d R} \alpha_{*}(\phi)=\alpha_{*}\left(\omega^{0}\right)$, and if $k=-1$ then $\left.\alpha(\Phi)\right|_{V^{\text {red }}}=0=\left.\alpha^{\prime}\left(\Phi^{\prime}\right)\right|_{V^{\text {red }}}$. Therefore Proposition 2.4(c) applies, yielding $\Psi \in B^{k}$ and $\psi \in\left(\Omega_{B}^{1}\right)^{k-1}$ with

$$
\begin{aligned}
\alpha(\Phi)-\alpha^{\prime}\left(\Phi^{\prime}\right) & =\mathrm{d} \Psi & & \text { in } B^{k+1}, \text { and } \\
\alpha_{*}(\phi)-\alpha_{*}^{\prime}\left(\phi^{\prime}\right) & =\mathrm{d}_{d R} \Psi+\mathrm{d} \psi & & \text { in }\left(\Omega_{B}^{1}\right)^{k} .
\end{aligned}
$$

The data $B, \boldsymbol{V}, \boldsymbol{i}, \alpha, \alpha^{\prime}, r, \Psi, \psi$ compare the Darboux presentations $A, \boldsymbol{U}$, $\boldsymbol{\varphi}, \omega, \Phi, \phi$ and $A^{\prime}, \boldsymbol{U}^{\prime}, \boldsymbol{\varphi}^{\prime}, \omega^{\prime}, \Phi^{\prime}, \phi^{\prime}$ for $\boldsymbol{X}$ near $q$.

Using this method in the case $k=-1$ yields the following comparison result for the critical atlases of Corollary 2.11 We have replaced $t_{0}(\boldsymbol{U}), U(0), t_{0}\left(\boldsymbol{U}^{\prime}\right)$, $U^{\prime}(0), t_{0}(\boldsymbol{V}), V(0)$, Spec $\alpha^{0}$, Spec $\alpha^{\prime 0}$ above by $T, U, T^{\prime}, U^{\prime}, R, V, \theta, \theta^{\prime}$. The conclusion $f \circ \theta-f^{\prime} \circ \theta^{\prime} \in I_{R, V}^{2}$ is proved as in [4, Ex. 5.35].

Proposition 2.13. Let $\left(\boldsymbol{X}, \omega_{\boldsymbol{X}}\right)$ be a-1-shifted symplectic derived Artin $\mathbb{K}$ stack, and $X=t_{0}(\boldsymbol{X})$ the corresponding classical Artin $\mathbb{K}$-stack. Suppose $U, f$ : $U \rightarrow \mathbb{A}^{1}, \varphi: T=\operatorname{Crit}(f) \rightarrow X$ and $U^{\prime}, f^{\prime}: U^{\prime} \rightarrow \mathbb{A}^{1}, \varphi^{\prime}: T^{\prime}=\operatorname{Crit}\left(f^{\prime}\right) \rightarrow X$ are two choices of the data constructed in Corollary 2.11 for points $p, p^{\prime} \in \boldsymbol{X}$, with $\left.f\right|_{T^{\mathrm{red}}}=0=\left.f^{\prime}\right|_{T^{\prime \mathrm{red}}}$. Let $q \in T \times_{\varphi, X, \varphi^{\prime}} T^{\prime}$. Then there exist a smooth $\mathbb{K}$-scheme $V$, a closed $\mathbb{K}$-subscheme $R \subseteq V$, a point $r \in R$, and morphisms 
$\theta: V \rightarrow U, \theta^{\prime}: V \rightarrow U^{\prime}$ with $\theta(R) \subseteq T, \theta^{\prime}(R) \subseteq T^{\prime}$ such that the following diagram 2-commutes (homotopy commutes) in $\operatorname{Art}_{\mathbb{K}}$ :

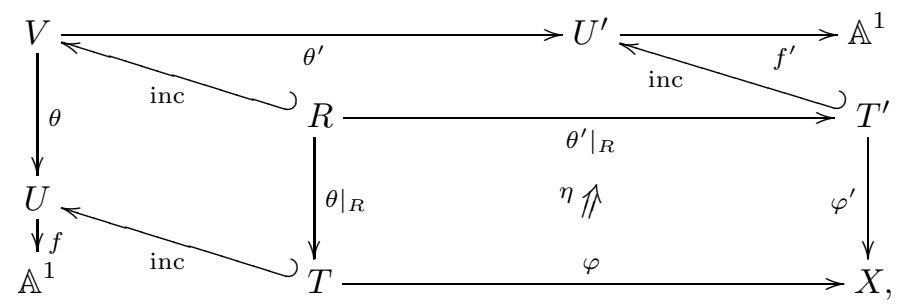

and the induced morphism $R \rightarrow T \times_{X} T^{\prime}$ is étale and maps $r \mapsto q$. Furthermore $f \circ \theta-f^{\prime} \circ \theta^{\prime} \in I_{R, V}^{2}$, where $I_{R, V} \subseteq \mathcal{O}_{V}$ is the ideal of functions vanishing on $R \subseteq V$.

\section{A truncation functor to d-critical stacks}

Section 3.1 summarizes the theory of algebraic d-critical loci (on classical $\mathbb{K}$ schemes) from [15], and the truncation functor from -1-shifted symplectic derived $\mathbb{K}$-schemes to algebraic d-critical loci from [4, §6]. Section 3.2 explains the generalization of d-critical loci to Artin stacks from [15, called d-critical stacks. Our main result Theorem [3.18, extending the truncation functor of [4, §6] to (derived) Artin stacks, is stated in $\$ 3.3$ and proved in 3.4

\subsection{Algebraic d-critical loci, the $\mathbb{K}$-scheme case}

We now review the main ideas and results in the last author's theory [15] of (algebraic) d-critical loci. Readers are referred to [15] for more details. Throughout $\mathbb{K}$ is an algebraically closed field with char $\mathbb{K} \neq 2$, though we will take char $\mathbb{K}=0$ in Theorem 3.18 and its corollaries.

Let $X$ be a $\mathbb{K}$-scheme. Then [15, Th. $2.1 \&$ Prop. 2.3] define a natural sheaf of $\mathbb{K}$-algebras $\mathcal{S}_{X}$ on $X$ in either the Zariski or étale topologies (we will use the étale version for the extension to Artin stacks), with the following properties:

(a) Suppose $R \subseteq X$ is Zariski open, $U$ is a smooth $\mathbb{K}$-scheme, and $i: R \hookrightarrow U$ a closed embedding. Define an ideal $I_{R, U} \subseteq i^{-1}\left(\mathcal{O}_{U}\right)$ by the exact sequence

$$
\left.0 \longrightarrow I_{R, U} \longrightarrow i^{-1}\left(\mathcal{O}_{U}\right) \stackrel{i^{\sharp}}{\longrightarrow} \mathcal{O}_{X}\right|_{R} \longrightarrow 0,
$$

where $\mathcal{O}_{X}, \mathcal{O}_{U}$ are the sheaves of regular functions on $X, U$. Then there is an exact sequence on $R$, where $\mathrm{d}: f+I_{R, U}^{2} \mapsto \mathrm{d} f+I_{R, U} \cdot i^{-1}\left(T^{*} U\right)$

$$
\left.0 \longrightarrow \mathcal{S}_{X}\right|_{R} \stackrel{\iota_{R, U}}{\longrightarrow} \frac{i^{-1}\left(\mathcal{O}_{U}\right)}{I_{R, U}^{2}} \longrightarrow \frac{\mathrm{d}}{\longrightarrow} \frac{i^{-1}\left(T^{*} U\right)}{I_{R, U} \cdot i^{-1}\left(T^{*} U\right)}
$$


(b) Let $R \subseteq S \subseteq X$ be Zariski open, $U, V$ be smooth $\mathbb{K}$-schemes, $i: R \hookrightarrow U$, $j: S \hookrightarrow V$ closed embeddings, and $\Phi: U \rightarrow V$ a morphism with $\Phi \circ i=$ $\left.j\right|_{R}: R \rightarrow V$. Then the following diagram of sheaves on $R$ commutes:

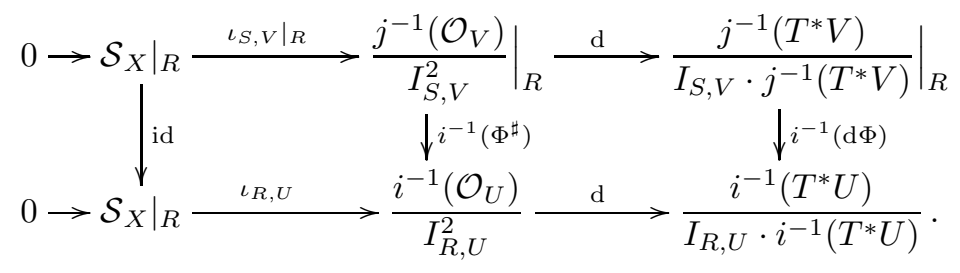

(c) There is a natural decomposition $\mathcal{S}_{X}=\mathcal{S}_{X}^{0} \oplus \mathbb{K}_{X}$, where $\mathbb{K}_{X}$ is the constant sheaf on $X$ with fibre $\mathbb{K}$, and $\mathcal{S}_{X}^{0} \subset \mathcal{S}_{X}$ is the kernel of the composition

$$
\mathcal{S}_{X} \longrightarrow \mathcal{O}_{X} \stackrel{i_{X}^{\sharp}}{\longrightarrow} \mathcal{O}_{X} \text { red },
$$

with $i_{X}: X^{\text {red }} \hookrightarrow X$ the reduced $\mathbb{K}$-subscheme of $X$.

(d) Let $\phi: X \rightarrow Y$ be a morphism of $\mathbb{K}$-schemes. Then there is a unique morphism $\phi^{\star}: \phi^{-1}\left(\mathcal{S}_{Y}\right) \rightarrow \mathcal{S}_{X}$ of sheaves of $\mathbb{K}$-algebras on $X$, which maps $\phi^{-1}\left(\mathcal{S}_{Y}^{0}\right) \rightarrow \mathcal{S}_{X}^{0}$, such that if $R \subseteq X, S \subseteq Y$ are Zariski open with $\phi(R) \subseteq S, U, V$ are smooth schemes, $i: R \hookrightarrow U, j: S \hookrightarrow V$ are closed embeddings, and $\Phi: U \rightarrow V$ is a morphism with $\Phi \circ i=\left.j \circ \phi\right|_{R}: R \rightarrow V$, then as for (3.1) the following diagram of sheaves on $R$ commutes:

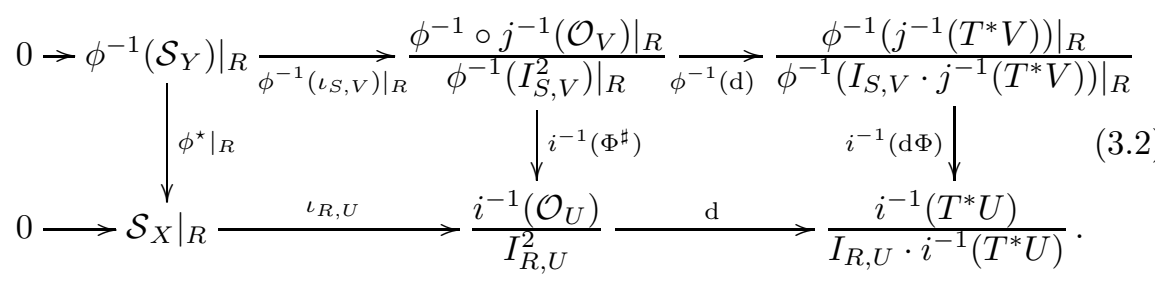

(e) If $X \stackrel{\phi}{\longrightarrow} Y \stackrel{\psi}{\longrightarrow} Z$ are smooth morphisms of $\mathbb{K}$-schemes, then

$$
(\psi \circ \phi)^{\star}=\phi^{\star} \circ \phi^{-1}\left(\psi^{\star}\right):(\psi \circ \phi)^{-1}\left(\mathcal{S}_{Z}\right)=\phi^{-1} \circ \psi^{-1}\left(\mathcal{S}_{Z}\right) \longrightarrow \mathcal{S}_{X} .
$$

If $\phi: X \rightarrow Y$ is $\operatorname{id}_{X}: X \rightarrow X$ then $\operatorname{id}_{X}^{\star}=\operatorname{id}_{\mathcal{S}_{X}}: \operatorname{id}_{X}^{-1}\left(\mathcal{S}_{X}\right)=\mathcal{S}_{X} \rightarrow \mathcal{S}_{X}$.

Following [15, Def. 2.5] we define algebraic d-critical loci:

Definition 3.1. An (algebraic) d-critical locus over a field $\mathbb{K}$ is a pair $(X, s)$, where $X$ is a $\mathbb{K}$-scheme and $s \in H^{0}\left(\mathcal{S}_{X}^{0}\right)$, such that for each $x \in X$, there exists a Zariski open neighbourhood $R$ of $x$ in $X$, a smooth $\mathbb{K}$-scheme $U$, a regular function $f: U \rightarrow \mathbb{A}^{1}=\mathbb{K}$, and a closed embedding $i: R \hookrightarrow U$, such that $i(R)=\operatorname{Crit}(f)$ as $\mathbb{K}$-subschemes of $U$, and $\iota_{R, U}\left(\left.s\right|_{R}\right)=i^{-1}(f)+I_{R, U}^{2}$. We call the quadruple $(R, U, f, i)$ a critical chart on $(X, s)$.

Let $(X, s)$ be an algebraic d-critical locus, and $(R, U, f, i)$ a critical chart on $(X, s)$. Let $U^{\prime} \subseteq U$ be Zariski open, and set $R^{\prime}=i^{-1}\left(U^{\prime}\right) \subseteq R, i^{\prime}=\left.i\right|_{R^{\prime}}: R^{\prime} \hookrightarrow$ 
$U^{\prime}$, and $f^{\prime}=\left.f\right|_{U^{\prime}}$. Then $\left(R^{\prime}, U^{\prime}, f^{\prime}, i^{\prime}\right)$ is a critical chart on $(X, s)$, and we call it a subchart of $(R, U, f, i)$. As a shorthand we write $\left(R^{\prime}, U^{\prime}, f^{\prime}, i^{\prime}\right) \subseteq(R, U, f, i)$.

Let $(R, U, f, i),(S, V, g, j)$ be critical charts on $(X, s)$, with $R \subseteq S \subseteq X$. An embedding of $(R, U, f, i)$ in $(S, V, g, j)$ is a locally closed embedding $\Phi: U \hookrightarrow V$ such that $\Phi \circ i=\left.j\right|_{R}$ and $f=g \circ \Phi$. As a shorthand we write $\Phi:(R, U, f, i) \hookrightarrow$ $(S, V, g, j)$. If $\Phi:(R, U, f, i) \hookrightarrow(S, V, g, j)$ and $\Psi:(S, V, g, j) \hookrightarrow(T, W, h, k)$ are embeddings, then $\Psi \circ \Phi:(R, U, i, e) \hookrightarrow(T, W, h, k)$ is also an embedding.

A morphism $\phi:(X, s) \rightarrow(Y, t)$ of d-critical loci $(X, s),(Y, t)$ is a $\mathbb{K}$-scheme morphism $\phi: X \rightarrow Y$ with $\phi^{\star}(t)=s$. This makes d-critical loci into a category.

There is also a complex analytic version, but we will not discuss it. Here are [15, Prop.s 2.8, 2.30, Th.s 2.20, 2.28, Def. 2.31, Rem 2.32 \& Cor. 2.33]:

Proposition 3.2. Let $\phi: X \rightarrow Y$ be a smooth morphism of $\mathbb{K}$-schemes. Suppose $t \in H^{0}\left(\mathcal{S}_{Y}^{0}\right)$, and set $s:=\phi^{\star}(t) \in H^{0}\left(\mathcal{S}_{X}^{0}\right)$. If $(Y, t)$ is a d-critical locus, then $(X, s)$ is a d-critical locus, and $\phi:(X, s) \rightarrow(Y, t)$ is a morphism of $d$ critical loci. Conversely, if also $\phi: X \rightarrow Y$ is surjective, then $(X, s)$ a d-critical locus implies $(Y, t)$ is a d-critical locus.

Theorem 3.3. Suppose $(X, s)$ is an algebraic d-critical locus, and $(R, U, f, i)$, $(S, V, g, j)$ are critical charts on $(X, s)$. Then for each $x \in R \cap S \subseteq X$ there exist subcharts $\left(R^{\prime}, U^{\prime}, f^{\prime}, i^{\prime}\right) \subseteq(R, U, f, i),\left(S^{\prime}, V^{\prime}, g^{\prime}, j^{\prime}\right) \subseteq(S, V, g, j)$ with $x \in R^{\prime} \cap S^{\prime} \subseteq X$, a critical chart $(T, W, h, k)$ on $(X, s)$, and embeddings $\Phi$ : $\left(R^{\prime}, U^{\prime}, f^{\prime}, i^{\prime}\right) \hookrightarrow(T, W, h, k), \Psi:\left(S^{\prime}, V^{\prime}, g^{\prime}, j^{\prime}\right) \hookrightarrow(T, W, h, k)$.

Theorem 3.4. Let $(X, s)$ be an algebraic d-critical locus, and $X^{\text {red }} \subseteq X$ the associated reduced $\mathbb{K}$-subscheme. Then there exists a line bundle $K_{X, s}$ on $X^{\mathrm{red}}$ which we call the canonical bundle of $(X, s)$, which is natural up to canonical isomorphism, and is characterized by the following properties:

(a) For each $x \in X^{\mathrm{red}}$, there is a canonical isomorphism

$$
\kappa_{x}:\left.K_{X, s}\right|_{x} \cong\left(\Lambda^{\text {top }} T_{x}^{*} X\right)^{\otimes^{2}}
$$

where $T_{x} X$ is the Zariski tangent space of $X$ at $x$.

(b) If $(R, U, f, i)$ is a critical chart on $(X, s)$, there is a natural isomorphism

$$
\iota_{R, U, f, i}:\left.\left.K_{X, s}\right|_{R^{\mathrm{red}}} \longrightarrow i^{*}\left(K_{U}^{\otimes^{2}}\right)\right|_{R^{\mathrm{red}}},
$$

where $K_{U}=\Lambda^{\operatorname{dim} U} T^{*} U$ is the canonical bundle of $U$ in the usual sense.

(c) In the situation of (b), let $x \in R$. Then we have an exact sequence

$$
0 \longrightarrow T_{x} X \stackrel{\left.\mathrm{d} i\right|_{x}}{\longrightarrow} T_{i(x)} U \stackrel{\operatorname{Hess}_{i(x)} f}{\longrightarrow} T_{i(x)}^{*} U \stackrel{\left.\mathrm{d} i\right|_{x} ^{*}}{\longrightarrow} T_{x}^{*} X \longrightarrow 0,
$$

and the following diagram commutes:

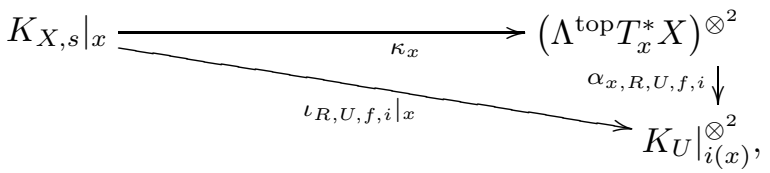

where $\alpha_{x, R, U, f, i}$ is induced by taking top exterior powers in (3.5). 
Proposition 3.5. Suppose $\phi:(X, s) \rightarrow(Y, t)$ is a morphism of d-critical loci with $\phi: X \rightarrow Y$ smooth, as in Proposition 3.2 . The relative cotangent bundle $T_{X / Y}^{*}$ is a vector bundle of mixed rank on $X$ in the exact sequence of coherent sheaves on $X$ :

$$
0 \longrightarrow \phi^{*}\left(T^{*} Y\right) \stackrel{\mathrm{d} \phi^{*}}{\longrightarrow} T^{*} X \longrightarrow T_{X / Y}^{*} \longrightarrow 0 .
$$

There is a natural isomorphism of line bundles on $X^{\mathrm{red}}$ :

$$
\Upsilon_{\phi}:\left.\left.\phi\right|_{X^{\text {red }}} ^{*}\left(K_{Y, t}\right) \otimes\left(\Lambda^{\mathrm{top}} T_{X / Y}^{*}\right)\right|_{X^{\text {red }}} ^{\otimes^{2}} \stackrel{\cong}{\longrightarrow} K_{X, s},
$$

such that for each $x \in X^{\text {red }}$ the following diagram of isomorphisms commutes:

$$
\begin{aligned}
& \left.\left.K_{Y, t}\right|_{\phi(x)} \otimes\left(\left.\Lambda^{\mathrm{top}} T_{X / Y}^{*}\right|_{x}\right)^{\otimes^{2}} \longrightarrow K_{X, s}\right|_{x} \\
& \downarrow^{\kappa_{\phi(x)} \otimes \mathrm{id}} \quad \kappa_{x} \downarrow \\
& \left(\Lambda^{\mathrm{top}} T_{\phi(x)}^{*} Y\right)^{\otimes^{2}} \otimes\left(\left.\Lambda^{\mathrm{top}} T_{X / Y}^{*}\right|_{x}\right)^{\otimes^{2} \longrightarrow} v_{x}^{\otimes^{2}} \longrightarrow\left(\Lambda^{\mathrm{top}} T_{x}^{*} X\right)^{\otimes^{2}},
\end{aligned}
$$

where $\kappa_{x}, \kappa_{\phi(x)}$ are as in (3.3), and $v_{x}:\left.\Lambda^{\text {top }} T_{\phi(x)}^{*} Y \otimes \Lambda^{\text {top }} T_{X / Y}^{*}\right|_{x} \rightarrow \Lambda^{\text {top }} T_{x}^{*} X$ is obtained by restricting (3.6) to $x$ and taking top exterior powers.

Definition 3.6. Let $(X, s)$ be an algebraic d-critical locus, and $K_{X, s}$ its canonical bundle from Theorem 3.4. An orientation on $(X, s)$ is a choice of square root line bundle $K_{X, s}^{1 / 2}$ for $K_{X, s}$ on $X^{\text {red }}$. That is, an orientation is a line bundle $L$ on $X^{\text {red }}$, together with an isomorphism $L^{\otimes^{2}}=L \otimes L \cong K_{X, s}$. A d-critical locus with an orientation will be called an oriented d-critical locus.

Remark 3.7. In view of equation (3.3), one might hope to define a canonical orientation $K_{X, s}^{1 / 2}$ for a d-critical locus $(X, s)$ by $\left.K_{X, s}^{1 / 2}\right|_{x}=\Lambda^{\text {top }} T_{x}^{*} X$ for $x \in X^{\text {red }}$. However, this does not work, as the spaces $\Lambda^{\text {top }} T_{x}^{*} X$ do not vary continuously with $x \in X^{\text {red }}$ if $X$ is not smooth. An example in [15, Ex. 2.39] shows that d-critical loci need not admit orientations.

In the situation of Proposition 3.5, the factor $\left.\left(\Lambda^{\text {top }} T_{X / Y}^{*}\right)\right|_{X^{\text {red }}} ^{\otimes^{2}}$ in (3.7) has a natural square $\left.\operatorname{root}\left(\Lambda^{\text {top }} T_{X / Y}^{*}\right)\right|_{X^{\text {red }}}$. Thus we deduce:

Corollary 3.8. Let $\phi:(X, s) \rightarrow(Y, t)$ be a morphism of d-critical loci with $\phi: X \rightarrow Y$ smooth. Then each orientation $K_{Y, t}^{1 / 2}$ for $(Y, t)$ lifts to a natural orientation $K_{X, s}^{1 / 2}=\left.\left.\phi\right|_{X^{\text {red }}} ^{*}\left(K_{Y, t}^{1 / 2}\right) \otimes\left(\Lambda^{\text {top }} T_{X / Y}^{*}\right)\right|_{X^{\text {red }}}$ for $(X, s)$.

The following result from [4 will be generalized to stacks in Theorem 3.18

Theorem 3.9 (Bussi, Brav and Joyce [4, Th. 6.6]). Suppose $(\boldsymbol{X}, \omega)$ is a-1shifted symplectic derived scheme in the sense of Pantev et al. 30. over an algebraically closed field $\mathbb{K}$ of characteristic zero, and let $X=t_{0}(\boldsymbol{X})$ be the associated classical $\mathbb{K}$-scheme of $\boldsymbol{X}$. Then $X$ extends naturally to an algebraic $d$-critical locus $(X, s)$. The canonical bundle $K_{X, s}$ from Theorem 3.4 is naturally isomorphic to the determinant line bundle $\left.\operatorname{det}\left(\mathbb{L}_{\boldsymbol{X}}\right)\right|_{X^{\text {red }}}$ of the cotangent complex $\mathbb{L}_{\boldsymbol{X}}$ of $\boldsymbol{X}$. 


\subsection{Extension to Artin stacks, and d-critical stacks}

In [15, §2.7-§2.8] we extend the material of \$3.1 from $\mathbb{K}$-schemes to Artin $\mathbb{K}$ stacks. We work in the context of the theory of sheaves on Artin stacks by Laumon and Moret-Bailly [21, $\S 12,13,15,18]$, including quasi-coherent, coherent, and constructible sheaves, and their derived categories. Unfortunately, Laumon and Moret-Bailly wrongly assume that 1-morphisms of algebraic stacks induce morphisms of lisse-étale topoi, so parts of their theory concerning pullbacks, etc., are unsatisfactory. Olsson 29] rewrites the theory, correcting this mistake. Laszlo and Olsson 22 24 study derived categories of constructible sheaves, and perverse sheaves, on Artin stacks, in more detail.

All of 21 24]29] work with sheaves on Artin stacks in the lisse-étale topology. We will not define these directly, but instead quote an alternative description from Laumon and Moret-Bailly 21] that we find more convenient.

Proposition 3.10 (Laumon and Moret-Bailly [21]). Let $X$ be an Artin $\mathbb{K}$-stack. The category of sheaves of sets on $X$ in the lisse-étale topology is equivalent to the category $\operatorname{Sh}(X)$ defined as follows:

(A) Objects $\mathcal{A}$ of $\operatorname{Sh}(X)$ comprise the following data:

(a) For each $\mathbb{K}$-scheme $T$ and smooth 1-morphism $t: T \rightarrow X$ in Art $_{\mathbb{K}}$, we are given a sheaf of sets $\mathcal{A}(T, t)$ on $T$, in the étale topology.

(b) For each 2-commutative diagram in $\mathrm{Art}_{\mathbb{K}}$ :

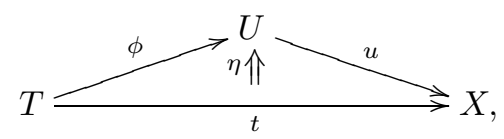

where $T, U$ are schemes and $t: T \rightarrow X, u: U \rightarrow X$ are smooth 1 morphisms in $\mathrm{Art}_{\mathbb{K}}$, we are given a morphism $\mathcal{A}(\phi, \eta): \phi^{-1}(\mathcal{A}(U, u)) \rightarrow$ $\mathcal{A}(T, t)$ of étale sheaves of sets on $T$.

This data must satisfy the following conditions:

(i) If $\phi: T \rightarrow U$ in (b) is étale, then $\mathcal{A}(\phi, \eta)$ is an isomorphism.

(ii) For each 2-commutative diagram in $\operatorname{Art}_{\mathbb{K}}$ :

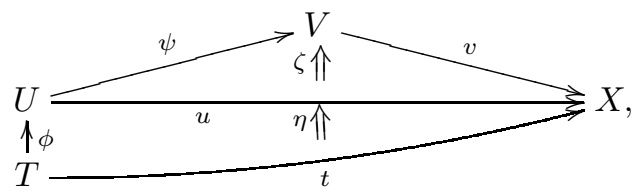

with $T, U, V$ schemes and $t, u, v$ smooth, we must have

$$
\begin{gathered}
\mathcal{A}\left(\psi \circ \phi,\left(\zeta * \operatorname{id}_{\phi}\right) \odot \eta\right)=\mathcal{A}(\phi, \eta) \circ \phi^{-1}(\mathcal{A}(\psi, \zeta)) \quad \text { as morphisms } \\
(\psi \circ \phi)^{-1}(\mathcal{A}(V, v))=\phi^{-1} \circ \psi^{-1}(\mathcal{A}(V, v)) \longrightarrow \mathcal{A}(T, t) .
\end{gathered}
$$


(B) Morphisms $\alpha: \mathcal{A} \rightarrow \mathcal{B}$ of $\operatorname{Sh}(X)$ comprise a morphism $\alpha(T, t): \mathcal{A}(T, t) \rightarrow$ $\mathcal{B}(T, t)$ of étale sheaves of sets on a scheme $T$ for all smooth 1-morphisms $t$ : $T \rightarrow X$, such that for each diagram (3.9) in (b) the following commutes:

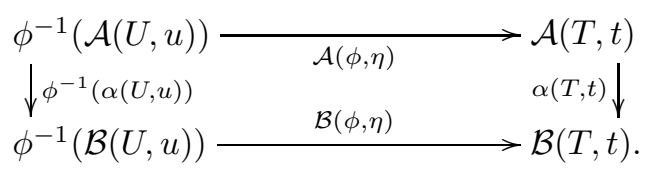

(C) Composition of morphisms $\mathcal{A} \stackrel{\alpha}{\longrightarrow} \mathcal{B} \stackrel{\beta}{\longrightarrow} \mathcal{C}$ in $\operatorname{Sh}(X)$ is $(\beta \circ \alpha)(T, t)=\beta(T, t)$ $\circ \alpha(T, t)$. Identity morphisms $\operatorname{id}_{\mathcal{A}}: \mathcal{A} \rightarrow \mathcal{A}$ are $\operatorname{id}_{\mathcal{A}}(T, t)=\operatorname{id}_{\mathcal{A}(T, t)}$.

The analogue of all the above also holds for (étale) sheaves of $\mathbb{K}$-vector spaces, sheaves of $\mathbb{K}$-algebras, and so on, in place of (étale) sheaves of sets.

Furthermore, the analogue of all the above holds for quasi-coherent sheaves, (or coherent sheaves, or vector bundles, or line bundles) on $X$, where in (a) $\mathcal{A}(T, t)$ becomes a quasi-coherent sheaf (or coherent sheaf, or vector bundle, or line bundle) on $T$, in (b) we replace $\phi^{-1}(\mathcal{A}(U, u))$ by the pullback $\phi^{*}(\mathcal{A}(U, u))$ of quasi-coherent sheaves (etc.), and $\mathcal{A}(\phi, \eta), \alpha(T, t)$ become morphisms of quasicoherent sheaves (etc.) on T.

We can also describe global sections of sheaves on Artin $\mathbb{K}$-stacks in the above framework: a global section $s \in H^{0}(\mathcal{A})$ of $\mathcal{A}$ in part (A) assigns a global section $s(T, t) \in H^{0}(\mathcal{A}(T, t))$ of $\mathcal{A}(T, t)$ on $T$ for all smooth $t: T \rightarrow X$ from a scheme $T$, such that $\mathcal{A}(\phi, \eta)^{*}(s(U, u))=s(T, t)$ in $H^{0}(\mathcal{A}(T, t))$ for all 2-commutative diagrams (3.9) with $t, u$ smooth.

In the rest of the paper we will use the notation of Proposition 3.10 for sheaves of all kinds on Artin $\mathbb{K}$-stacks. In [15, Cor. 2.52] we generalize the sheaves $\mathcal{S}_{X}, \mathcal{S}_{X}^{0}$ in 3.1 to Artin $\mathbb{K}$-stacks:

Proposition 3.11. Let $X$ be an Artin $\mathbb{K}$-stack, and write $\operatorname{Sh}(X)_{\mathbb{K} \text {-alg and }}$ $\operatorname{Sh}(X)_{\mathbb{K} \text {-vect }}$ for the categories of sheaves of $\mathbb{K}$-algebras and $\mathbb{K}$-vector spaces on $X$ defined in Proposition 3.10 , Then:

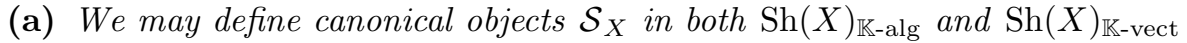
by $\mathcal{S}_{X}(T, t):=\mathcal{S}_{T}$ for all smooth morphisms $t: T \rightarrow X$ for $T \in \mathrm{Sch}_{\mathbb{K}}$, for $\mathcal{S}_{T}$ as in 3.1 taken to be a sheaf of $\mathbb{K}$-algebras (or $\mathbb{K}$-vector spaces) on $T$ in the étale topology, and $\mathcal{S}_{X}(\phi, \eta):=\phi^{\star}: \phi^{-1}\left(\mathcal{S}_{X}(U, u)\right)=\phi^{-1}\left(\mathcal{S}_{U}\right) \rightarrow$ $\mathcal{S}_{T}=\mathcal{S}_{X}(T, t)$ for all 2-commutative diagrams (3.9) in $\operatorname{Art}_{\mathbb{K}}$ with $t, u$ smooth, where $\phi^{\star}$ is as in 3.1 .

(b) There is a natural decomposition $\mathcal{S}_{X}=\mathbb{K}_{X} \oplus \mathcal{S}_{X}^{0}$ in $\operatorname{Sh}(X)_{\mathbb{K} \text {-vect }}$ induced by the splitting $\mathcal{S}_{X}(T, t)=\mathcal{S}_{T}=\mathbb{K}_{T} \oplus \mathcal{S}_{T}^{0}$ in $\$ 3.1$, where $\mathbb{K}_{X}$ is a sheaf of

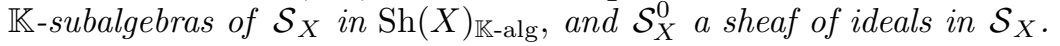

Here [15, Def. 2.53] is the generalization of Definition 3.1 to Artin stacks.

Definition 3.12. A $d$-critical stack $(X, s)$ is an Artin $\mathbb{K}$-stack $X$ and a global section $s \in H^{0}\left(\mathcal{S}_{X}^{0}\right)$, where $\mathcal{S}_{X}^{0}$ is as in Proposition 3.11, such that $(T, s(T, t))$ is an algebraic d-critical locus in the sense of Definition 3.1 for all smooth morphisms $t: T \rightarrow X$ with $T \in \mathrm{Sch}_{\mathbb{K}}$. 
In [15, Prop. 2.54] we give a convenient way to understand d-critical stacks $(X, s)$ in terms of d-critical structures on an atlas $t: T \rightarrow X$ for $X$.

Proposition 3.13. Suppose we are given a 2-commutative diagram in $\mathrm{Art}_{\mathbb{K}}$ :

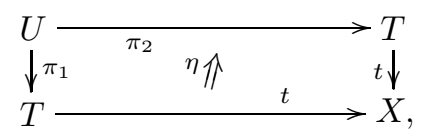

where $X$ is an Artin $\mathbb{K}$-stack, $T, U$ are $\mathbb{K}$-schemes, $t, \pi_{1}, \pi_{2}$ are smooth 1morphisms, $t: T \rightarrow X$ is surjective, and the 1-morphism $U \rightarrow T \times_{t, X, t} T$ induced by (3.10) is surjective. For instance, this happens if $U \rightrightarrows T$ is a groupoid in $\mathbb{K}$-schemes, and $X=[U \rightrightarrows T]$ the associated groupoid stack. Then:

(i) Let $\mathcal{S}_{X}$ be as in Proposition 3.11, and $\mathcal{S}_{T}, \mathcal{S}_{U}$ be as in r3.1 regarded as sheaves on $T, U$ in the étale topology, and define $\pi_{i}^{\star}: \pi_{i}^{-1}\left(\mathcal{S}_{T}\right) \rightarrow \mathcal{S}_{U}$ as in 3.1 for $i=1,2$. Consider the map $t^{*}: H^{0}\left(\mathcal{S}_{X}\right) \rightarrow H^{0}\left(\mathcal{S}_{T}\right)$ mapping $t^{*}: s \mapsto s(T, t)$. This is injective, and induces a bijection

$$
t^{*}: H^{0}\left(\mathcal{S}_{X}\right) \stackrel{\cong}{\longrightarrow}\left\{s^{\prime} \in H^{0}\left(\mathcal{S}_{T}\right): \pi_{1}^{\star}\left(s^{\prime}\right)=\pi_{2}^{\star}\left(s^{\prime}\right) \text { in } H^{0}\left(\mathcal{S}_{U}\right)\right\} .
$$

The analogue holds for $\mathcal{S}_{X}^{0}, \mathcal{S}_{T}^{0}, \mathcal{S}_{U}^{0}$.

(ii) Suppose $s \in H^{0}\left(\mathcal{S}_{X}^{0}\right)$, so that $t^{*}(s) \in H^{0}\left(\mathcal{S}_{T}^{0}\right)$ with $\pi_{1}^{\star} \circ t^{*}(s)=\pi_{2}^{\star} \circ t^{*}(s)$. Then $(X, s)$ is a d-critical stack if and only if $\left(T, t^{*}(s)\right)$ is an algebraic $d$-critical locus, and then $\left(U, \pi_{1}^{\star} \circ t^{*}(s)\right)$ is also an algebraic $d$-critical locus.

In [15, Ex. 2.55] we consider quotient stacks $X=[T / G]$.

Example 3.14. Suppose an algebraic $\mathbb{K}$-group $G$ acts on a $\mathbb{K}$-scheme $T$ with action $\mu: G \times T \rightarrow T$, and write $X$ for the quotient Artin $\mathbb{K}$-stack $[T / G]$. Then as in (3.10) there is a natural 2-Cartesian diagram

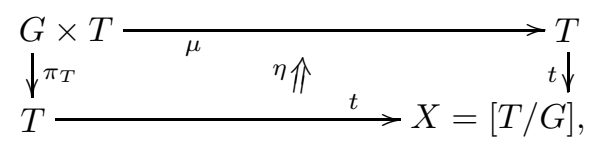

where $t: T \rightarrow X$ is a smooth atlas for $X$. If $s^{\prime} \in H^{0}\left(\mathcal{S}_{T}^{0}\right)$ then $\pi_{1}^{\star}\left(s^{\prime}\right)=\pi_{2}^{\star}\left(s^{\prime}\right)$ in (3.11) becomes $\pi_{T}^{\star}\left(s^{\prime}\right)=\mu^{\star}\left(s^{\prime}\right)$ on $G \times T$, that is, $s^{\prime}$ is $G$-invariant. Hence, Proposition 3.13 shows that d-critical structures $s$ on $X=[T / G]$ are in 1-1 correspondence with $G$-invariant d-critical structures $s^{\prime}$ on $T$.

Here [15, Th. 2.56] is an analogue of Theorem 3.4

Theorem 3.15. Let $(X, s)$ be a d-critical stack. Using the description of quasicoherent sheaves on $X^{\mathrm{red}}$ in Proposition 3.10 there is a line bundle $K_{X, s}$ on the reduced $\mathbb{K}$-substack $X^{\text {red }}$ of $X$ called the canonical bundle of $(X, s)$, unique up to canonical isomorphism, such that: 
(a) For each point $x \in X^{\text {red }} \subseteq X$ we have a canonical isomorphism

$$
\kappa_{x}:\left.K_{X, s}\right|_{x} \cong\left(\Lambda^{\mathrm{top}} T_{x}^{*} X\right)^{\otimes^{2}} \otimes\left(\Lambda^{\mathrm{top}} \mathfrak{I s o} \mathfrak{s}_{x}(X)\right)^{\otimes^{2}},
$$

where $T_{x}^{*} X$ is the Zariski cotangent space of $X$ at $x$, and $\mathfrak{I s o}_{x}(X)$ the Lie algebra of the isotropy group (stabilizer group) $\operatorname{Iso}_{x}(X)$ of $X$ at $x$.

(b) If $T$ is a $\mathbb{K}$-scheme and $t: T \rightarrow X$ a smooth 1-morphism, so that $t^{\text {red }}$ : $T^{\mathrm{red}} \rightarrow X^{\mathrm{red}}$ is also smooth, then there is a natural isomorphism of line bundles on $T^{\mathrm{red}}$ :

$$
\Gamma_{T, t}:\left.K_{X, s}\left(T^{\mathrm{red}}, t^{\mathrm{red}}\right) \stackrel{\cong}{\longrightarrow} K_{T, s(T, t)} \otimes\left(\Lambda^{\mathrm{top}} T_{T / X}^{*}\right)\right|_{T^{\mathrm{red}}} ^{\otimes^{-2}} .
$$

Here $(T, s(T, t))$ is an algebraic d-critical locus by Definition 3.12 and $K_{T, s(T, t)} \rightarrow T^{\mathrm{red}}$ is its canonical bundle from Theorem 3.4 .

(c) If $t: T \rightarrow X$ is a smooth 1-morphism, we have a distinguished triangle in $D_{\mathrm{qcoh}}(T)$ :

$$
t^{*}\left(\mathbb{L}_{X}\right) \stackrel{\mathbb{L}_{t}}{\longrightarrow} \mathbb{L}_{T} \longrightarrow T_{T / X}^{*} \longrightarrow t^{*}\left(\mathbb{L}_{X}\right)[1],
$$

where $\mathbb{L}_{T}, \mathbb{L}_{X}$ are the cotangent complexes of $T, X$, and $T_{T / X}^{*}$ the relative cotangent bundle of $t: T \rightarrow X$, a vector bundle of mixed rank on $T$. Let $p \in T^{\mathrm{red}} \subseteq T$, so that $t(p):=t \circ p \in X$. Taking the long exact cohomology sequence of (3.14) and restricting to $p \in T$ gives an exact sequence

$$
\left.0 \longrightarrow T_{t(p)}^{*} X \longrightarrow T_{p}^{*} T \longrightarrow T_{T / X}^{*}\right|_{p} \longrightarrow \mathfrak{I s o c}_{t(p)}(X)^{*} \longrightarrow 0 .
$$

Then the following diagram commutes:

$$
\begin{aligned}
& \left.K_{X, s}\right|_{t(p)}=\left.\left.\left.K_{X, s}\left(T^{\mathrm{red}}, t^{\mathrm{red}}\right)\right|_{p} \underset{\left.\Gamma_{T, t}\right|_{p}}{\longrightarrow} K_{T, s(T, t)}\right|_{p} \otimes\left(\Lambda^{\mathrm{top}} T_{T / X}^{*}\right)\right|_{p} ^{\otimes^{-2}} \\
& \quad \kappa_{t(p)} \\
& \left.\left(\Lambda^{\mathrm{top}} T_{t(p)}^{*} X\right)^{\otimes^{2}} \otimes\left(\Lambda^{\mathrm{top}} \mathfrak{I} \mathfrak{j o} \mathfrak{s}_{t(p)}(X)\right)^{\otimes^{2}} \stackrel{\alpha_{p}^{2}}{\rightarrow}\left(\Lambda^{\mathrm{top}} T_{p}^{*} T\right)^{\otimes^{2}} \otimes\left(\Lambda^{\mathrm{top}} T_{T / X}^{*}\right)\right|_{p} ^{\otimes^{-2}},
\end{aligned}
$$

where $\kappa_{p}, \kappa_{t(p)}, \Gamma_{T, t}$ are as in (3.3), (3.12) and (3.13), respectively, and $\alpha_{p}:\left.\Lambda^{\text {top }} T_{t(p)}^{*} X \otimes \Lambda^{\text {top }} \mathfrak{I s o}_{t(p)}(X) \stackrel{\cong}{\longrightarrow} \Lambda^{\text {top }} T_{p}^{*} T \otimes \Lambda^{\text {top }} T_{T / X}^{*}\right|_{p} ^{-1}$ is induced by taking top exterior powers in (3.15).

Here [15, Def. 2.57] is the analogue of Definition 3.6

Definition 3.16. Let $(X, s)$ be a d-critical stack, and $K_{X, s}$ its canonical bundle from Theorem 3.15. An orientation on $(X, s)$ is a choice of square root line bundle $K_{X, s}^{1 / 2}$ for $K_{X, s}$ on $X^{\text {red }}$. That is, an orientation is a line bundle $L$ on $X^{\text {red }}$, together with an isomorphism $L^{\otimes^{2}}=L \otimes L \cong K_{X, s}$. A d-critical stack with an orientation will be called an oriented d-critical stack.

Let $(X, s)$ be an oriented d-critical stack. Then for each smooth $t: T \rightarrow$ $X$ we have a square root $K_{X, s}^{1 / 2}\left(T^{\text {red }}, t^{\text {red }}\right)$. Thus by $(3.13), K_{X, s}^{1 / 2}\left(T^{\text {red }}, t^{\text {red }}\right) \otimes$ $\left.\left(\Lambda^{\text {top }} \mathbb{L}_{T / X}\right)\right|_{T^{\text {red }}}$ is a square root for $K_{T, s(T, t)}$. This proves [15, Lem. 2.58]: 
Lemma 3.17. Let $(X, s)$ be a d-critical stack. Then an orientation $K_{X, s}^{1 / 2}$ for $(X, s)$ determines a canonical orientation $K_{T, s(T, t)}^{1 / 2}$ for the algebraic d-critical locus $(T, s(T, t))$, for all smooth $t: T \rightarrow X$ with $T$ a $\mathbb{K}$-scheme.

\subsection{From -1-shifted symplectic stacks to d-critical stacks}

Here is the main result of this section, the analogue of Theorem 3.9 from [4].

Theorem 3.18. Let $\mathbb{K}$ be an algebraically closed field of characteristic zero, $\left(\boldsymbol{X}, \omega_{\boldsymbol{X}}\right)$ a-1-shifted symplectic derived Artin $\mathbb{K}$-stack, and $X=t_{0}(\boldsymbol{X})$ the corresponding classical Artin $\mathbb{K}$-stack. Then there exists a unique $d$-critical structure $s \in H^{0}\left(\mathcal{S}_{X}^{0}\right)$ on $X$, making $(X, s)$ into a d-critical stack, with the following properties:

(a) Let $U, f: U \rightarrow \mathbb{A}^{1}, T=\operatorname{Crit}(f)$ and $\varphi: T \rightarrow X$ be as in Corollary 2.11 with $\left.f\right|_{T^{\mathrm{red}}}=0$. As in 3.1 , there is a unique $s_{T} \in H^{0}\left(\mathcal{S}_{T}^{0}\right)$ on $T$ with $\iota_{T, U}\left(s_{T}\right)=i^{-1}(f)+I_{T, U}^{2}$, and $\left(T, s_{T}\right)$ is an algebraic d-critical locus. Then $s(T, \varphi)=s_{T}$ in $H^{0}\left(\mathcal{S}_{T}^{0}\right)$.

(b) The canonical bundle $K_{X, s}$ of $(X, s)$ from Theorem 3.15 is naturally isomorphic to the restriction $\left.\operatorname{det}\left(\mathbb{L}_{\boldsymbol{X}}\right)\right|_{X^{\text {red }}}$ to $X^{\text {red }} \subseteq X \subseteq X$ of the determinant line bundle $\operatorname{det}\left(\mathbb{L}_{\boldsymbol{X}}\right)$ of the cotangent complex $\mathbb{L}_{\boldsymbol{X}}$ of $\boldsymbol{X}$.

We can think of Theorem 3.18 as defining a truncation functor

$$
\begin{gathered}
F:\left\{\infty \text {-category of }-1 \text {-shifted symplectic derived } \operatorname{Artin} \mathbb{K} \text {-stacks }\left(\boldsymbol{X}, \omega_{\boldsymbol{X}}\right)\right\} \\
\longrightarrow\{2 \text {-category of d-critical stacks }(X, s) \text { over } \mathbb{K}\} .
\end{gathered}
$$

Let $Y$ be a Calabi-Yau 3-fold over $\mathbb{K}$, and $\mathcal{M}$ a classical moduli $\mathbb{K}$-stack of coherent sheaves in $\operatorname{coh}(Y)$, or complexes of coherent sheaves in $D^{b} \operatorname{coh}(Y)$. There is a natural obstruction theory $\phi: \mathcal{E}^{\bullet} \rightarrow \mathbb{L}_{\mathcal{M}}$ on $\mathcal{M}$, where $\mathcal{E}^{\bullet} \in D_{\text {qcoh }}(\mathcal{M})$ is perfect in the interval $[-2,1]$, and $\left.h^{i}\left(\mathcal{E}^{\bullet}\right)\right|_{F} \cong \operatorname{Ext}^{1-i}(F, F)^{*}$ for each $\mathbb{K}$-point $F \in \mathcal{M}$, regarding $F$ as an object in $\operatorname{coh}(Y)$ or $D^{b} \operatorname{coh}(Y)$. Now in derived algebraic geometry $\mathcal{M}=t_{0}(\mathcal{M})$ for $\mathcal{M}$ the corresponding derived moduli $\mathbb{K}$ stack, and $\phi: \mathcal{E}^{\bullet} \rightarrow \mathbb{L}_{\mathcal{M}}$ is $\mathbb{L}_{t_{0}}:\left.\mathbb{L}_{\mathcal{M}}\right|_{\mathcal{M}} \rightarrow \mathbb{L}_{\mathcal{M}}$. Pantev et al. [30, §2.1] prove $\mathcal{M}$ has a -1 -shifted symplectic structure $\omega$. Thus Theorem 3.18 implies:

Corollary 3.19. Suppose $Y$ is a Calabi-Yau 3-fold over $\mathbb{K}$ of characteristic zero, and $\mathcal{M}$ a classical moduli $\mathbb{K}$-stack of coherent sheaves $F$ in $\operatorname{coh}(Y)$, or complexes of coherent sheaves $F^{\bullet}$ in $D^{b} \operatorname{coh}(Y)$ with $\operatorname{Ext}^{<0}\left(F^{\bullet}, F^{\bullet}\right)=0$, with obstruction theory $\phi: \mathcal{E}^{\bullet} \rightarrow \mathbb{L}_{\mathcal{M}}$. Then $\mathcal{M}$ extends naturally to an algebraic $d$-critical locus $(\mathcal{M}, s)$. The canonical bundle $K_{\mathcal{M}, s}$ from Theorem 3.15 is naturally isomorphic to $\left.\operatorname{det}\left(\mathcal{E}^{\bullet}\right)\right|_{\mathcal{M}^{\text {red }}}$. 


\subsection{Proof of Theorem 3.18}

Let $\left(\boldsymbol{X}, \omega_{\boldsymbol{X}}\right)$ be a -1 -shifted symplectic derived Artin $\mathbb{K}$-stack, with char $\mathbb{K}=0$, and $X=t_{0}(\boldsymbol{X})$. For each $p \in X$, Corollary 2.11 gives data $T=\operatorname{Crit}(f: U \rightarrow$ $\mathbb{A}^{1}$ ) with $\left.f\right|_{T^{\text {red }}}=0, t \in T$ and a smooth $\varphi: T \rightarrow X$ with $\varphi(t)=p$. Choose $U_{j}, f_{j}, T_{j}, \varphi_{j}$ from Corollary 2.11 for $j$ in an indexing set $J$, such that $\coprod_{j \in J} \varphi_{j}$ : $\coprod_{j \in J} T_{j} \rightarrow X$ is surjective. Then $\coprod_{j \in J} \varphi_{j}: \coprod_{j \in J} T_{j} \rightarrow X$ is a smooth atlas for $X$. As in $\$ 3.1$ there is a unique $s_{j} \in H^{0}\left(\mathcal{S}_{T_{j}}^{0}\right)$ with $\iota_{T_{j}, U_{j}}\left(s_{j}\right)=i_{j}^{-1}\left(f_{j}\right)+I_{T_{j}, U_{j}}^{2}$, and $\left(T_{j}, s_{j}\right)$ is an algebraic d-critical locus for each $j \in J$.

Let $j, k \in J$, and $q \in T_{j} \times_{\varphi_{j}, X, \varphi_{k}} T_{k}$. Applying Proposition 2.13 gives a smooth $\mathbb{K}$-scheme $V_{j k}$, a closed $\mathbb{K}$-subscheme $R_{j k} \subseteq V_{j k}$, a point $r \in R_{j k}$, and morphisms $\theta_{j k}: V_{j k} \rightarrow U_{j}, \theta_{j k}^{\prime}: V_{j k} \rightarrow U_{k}$ with $\theta_{j k}\left(R_{j k}\right) \subseteq T_{j}, \theta_{j k}^{\prime}\left(R_{j k}\right) \subseteq T_{k}$, such that the following diagram 2-commutes in $\operatorname{Art}_{\mathbb{K}}$ :

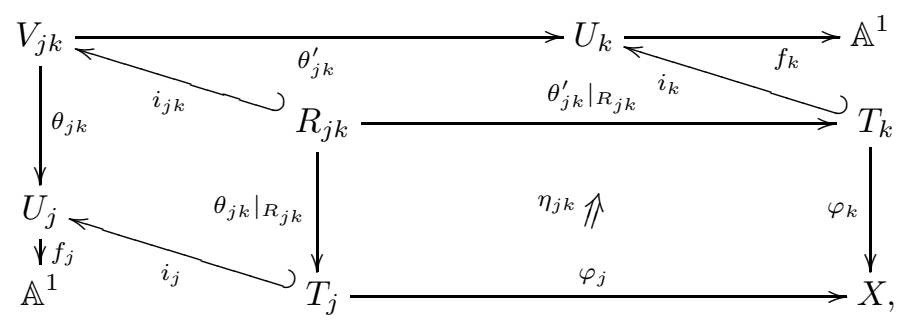

where $i_{j}, i_{k}, i_{j k}$ are the inclusions, and the induced morphism $R_{j k} \rightarrow T_{j} \times_{X} T_{k}$ is étale and maps $r \mapsto q$, and $f_{j} \circ \theta_{j k}-f_{k} \circ \theta_{j k}^{\prime} \in I_{R_{j k}, V_{j k}}^{2}$.

As we can do this for each $q \in T_{j} \times_{X} T_{k}$, we can choose a family of such $V_{j k}^{l}, R_{j k}^{l}, \theta_{j k}^{l}, \theta_{j k}^{\prime l}, \eta_{j k}^{l}, i_{j k}^{l}$ for $l \in K_{j k}$, where $K_{j k}$ is an indexing set, such that the induced morphism $\coprod_{l \in K_{j k}} R_{j k}^{l} \rightarrow T_{j} \times_{X} T_{k}$ is étale and surjective. We apply Proposition 3.13 to the 2 -commutative diagram

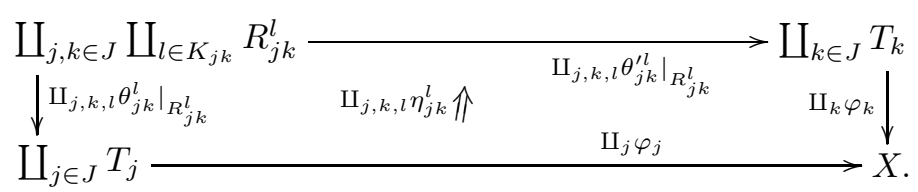

Here $\coprod_{j} \varphi_{j}: \coprod_{j} T_{j} \rightarrow X$ is smooth and surjective, and $\coprod_{j, k . l} R_{j k}^{l} \rightarrow \coprod_{j, k} T_{j} \times_{X}$ $T_{k}$ étale and surjective, so the hypotheses of Proposition 3.13 hold.

Now for all $j, k \in J$ and $l \in K_{j k}$, in the notation of 3.1, we have

$$
\begin{aligned}
& \left.\iota_{R_{j k}^{l}, V_{j k}^{l}} \circ \theta_{j k}^{l}\right|_{R_{j k}^{l}} ^{\star}\left(s_{j}\right)=\left.\left(i_{j k}^{l}\right)^{-1}\left(\theta_{j k}^{l \sharp}\right) \circ \theta_{j k}^{l}\right|_{R_{j k}^{l}} ^{-1}\left(\iota_{T_{j}, U_{j}}\left(s_{j}\right)\right) \\
& =\left.\theta_{j k}^{l}\right|_{R_{j k}^{l}} ^{-1}\left(i_{j}^{-1}\left(f_{j}\right)+I_{T_{j}, U_{j}}^{2}\right)=\left(i_{j k}^{l}\right)^{-1}\left(f_{j} \circ \theta_{j k}^{l}+I_{R_{j k}^{l}, V_{j k}^{l}}^{2}\right) \\
& =\left(i_{j k}^{l}\right)^{-1}\left(f_{k} \circ \theta_{j k}^{l \prime}+I_{R_{j k}^{l}, V_{j k}^{l}}^{2}\right)=\left.\theta_{j k}^{\prime l}\right|_{R_{j k}^{l}} ^{-1}\left(i_{k}^{-1}\left(f_{k}\right)+I_{T_{k}, U_{k}}^{2}\right) \\
& =\left.\left(i_{j k}^{l}\right)^{-1}\left(\theta_{j k}^{\prime l \sharp}\right) \circ \theta_{j k}^{\prime l}\right|_{R_{j k}^{l}} ^{-1}\left(\iota_{T_{k}, U_{k}}\left(s_{k}\right)\right)=\left.\iota_{R_{j k}^{l}, V_{j k}^{l}} \circ \theta_{j k}^{\prime l}\right|_{R_{j k}^{l}} ^{\star}\left(s_{k}\right),
\end{aligned}
$$


using (3.2) in the first and seventh steps, the definitions of $s_{j}, s_{k}$ in the second and sixth, and $f_{j} \circ \theta_{j k}^{l}-f_{k} \circ \theta_{j k}^{\prime l} \in I_{R_{j k}^{l}, V_{j k}^{l}}^{2}$ in the fourth. As $\iota_{R_{j k}^{l}, V_{j k}^{l}}$ is injective, this implies that $\left.\theta_{j k}^{l}\right|_{R_{j k}^{l}} ^{\star}\left(s_{j}\right)=\left.\theta_{j k}^{\prime l}\right|_{R_{j k}^{l}} ^{\star}\left(s_{k}\right)$ in $H^{0}\left(\mathcal{S}_{R_{j k}^{l}}^{0}\right)$. Since this holds for all $j, k, l$, we see that $\left(\left.\coprod_{j, k, l} \theta_{j k}^{l}\right|_{R_{j k}^{l}}\right)^{\star}\left(\coprod_{j} s_{j}\right)=\left(\left.\coprod_{j, k, l} \theta_{j k}^{\prime l}\right|_{R_{j k}^{l}}\right)^{\star}\left(\coprod_{k} s_{k}\right)$ in $H^{0}\left(\mathcal{S}_{\amalg_{j, k}, l}^{0} R_{j k}^{l}\right)$. Therefore Proposition 3.13(i) shows that there exists a unique $s \in H^{0}\left(\mathcal{S}_{X}\right)$ with $s\left(\coprod_{j} T_{j}, \coprod_{j} \varphi_{j}\right)=\coprod_{j} s_{j}$, that is, with $s\left(T_{j}, \varphi_{j}\right)=s_{j}$ for all $j \in J$. Also, as $\left(\coprod_{j} T_{j}, \coprod_{j} s_{j}\right)$ is an algebraic d-critical locus, Proposition 3.13(ii) shows that $(X, s)$ is a d-critical stack.

To show $s \in H^{0}\left(\mathcal{S}_{X}\right)$ is independent of the choice of data $J, U_{j}, f_{j}, T_{j}, \varphi_{j}$, $K_{j k}, V_{j k}^{l}, R_{j k}^{l}, \theta_{j k}^{l}, \theta_{j k}^{\prime l}, \eta_{j k}^{l}, i_{j k}^{l}$, suppose $J^{\prime}, U_{j^{\prime}}^{\prime}, f_{j^{\prime}}^{\prime}, \ldots$ is another set of choices yielding $s^{\prime} \in H^{0}\left(\mathcal{S}_{X}\right)$ with $s^{\prime}\left(T_{j^{\prime}}^{\prime}, \varphi_{j^{\prime}}^{\prime}\right)=s_{j^{\prime}}^{\prime}$ for all $j^{\prime} \in J^{\prime}$. Applying the same argument with $J^{\prime \prime}=J \amalg J^{\prime}$ and data $U_{j}, f_{j}, T_{j}, \varphi_{j}, j \in J$ and $U_{j^{\prime}}^{\prime}, f_{j^{\prime}}^{\prime}, T_{j^{\prime}}^{\prime}, \varphi_{j^{\prime}}^{\prime}$, $j^{\prime} \in J^{\prime}$, with $K_{j k}^{\prime \prime}=K_{j k}, V_{j k}^{\prime \prime l}=V_{j k}^{l}, \ldots$ for $j, k \in J \subset J^{\prime \prime}$, and $K_{j^{\prime} k^{\prime}}^{\prime \prime}=K_{j^{\prime} k^{\prime}}^{\prime}$, $V_{j^{\prime} k^{\prime}}^{\prime \prime l}=V_{j^{\prime} k^{\prime}}^{\prime l}, \ldots$ for $j^{\prime}, k^{\prime} \in J^{\prime} \subset J^{\prime \prime}$, and the remaining $K_{j k}^{\prime \prime}, V_{j k}^{\prime \prime l}, \ldots$ arbitrary, yields a third section $s^{\prime \prime} \in H^{0}\left(\mathcal{S}_{X}\right)$ satisfying $s^{\prime \prime}\left(T_{j}, \varphi_{j}\right)=s_{j}$ for all $j \in J$ and $s^{\prime \prime}\left(T_{j^{\prime}}^{\prime}, \varphi_{j^{\prime}}^{\prime}\right)=s_{j^{\prime}}^{\prime}$ for all $j^{\prime} \in J^{\prime}$. So the uniqueness property of $s, s^{\prime}$ gives $s=s^{\prime \prime}=s^{\prime}$, and $s$ is independent of the choice of data $J, U_{j}, f_{j}, \ldots$

Let $U, f: U \rightarrow \mathbb{A}^{1}, T=\operatorname{Crit}(f)$ and $\varphi: T \rightarrow X$ be as in Corollary 2.11, with $\left.f\right|_{T^{\text {red }}}=0$. By defining $s \in H^{0}\left(\mathcal{S}_{X}\right)$ above using data $J, U_{j}, f_{j}, \ldots$ chosen such that $U_{j}=U, f_{j}=f, T_{j}=T, \varphi_{j}=\varphi$ for some $j \in J$, which is allowed as $s$ is independent of this choice, we see that $s(T, \varphi)=s_{T}$ in $H^{0}\left(\mathcal{S}_{T}^{0}\right)$. This proves Theorem 3.18(a).

For part (b), let $U, f: U \rightarrow \mathbb{A}^{1}, T=\operatorname{Crit}(f)$ and $\varphi: T \rightarrow X$ be as in Corollary 2.11] with $i: T \hookrightarrow U$ the inclusion, so that $s(T, \varphi)=s_{T}$ in $H^{0}\left(\mathcal{S}_{T}^{0}\right)$ with $\iota_{T, U}\left(s_{T}\right)=i^{-1}(f)+I_{T, U}^{2}$ by (a). Then $(T, U, f, i)$ is a critical chart on the algebraic d-critical locus $\left(T, s_{T}\right)$, so Theorem 3.4(b) gives an isomorphism

$$
\iota_{T, U, f, i}:\left.K_{T, s_{T}} \longrightarrow i^{*}\left(K_{U}^{\otimes^{2}}\right)\right|_{T^{\mathrm{red}}}
$$

The data in Corollary 2.11 come from Theorem 2.10(a),(b) with $k=-1$, but with different notation. To distinguish the two, we write " $"$ over notation from Theorem 2.10. Then Theorem 2.10(a),(b) give affine derived $\mathbb{K}$-schemes $\check{\boldsymbol{U}}, \check{\boldsymbol{V}}$, a -1 -shifted symplectic structure $\check{\omega}_{B}$ on $\check{\boldsymbol{V}}$, and morphisms $\check{\boldsymbol{i}}: \check{\boldsymbol{U}} \rightarrow \check{\boldsymbol{V}}$, $\check{\boldsymbol{\varphi}}: \check{\boldsymbol{U}} \rightarrow \boldsymbol{X}$ such that $\check{\boldsymbol{\varphi}}^{*}\left(\omega_{\boldsymbol{X}}\right) \sim \check{\boldsymbol{i}}^{*}\left(\check{\omega}_{B}\right)$, and $\check{\imath}=t_{0}(\check{\boldsymbol{i}}): \check{U}=t_{0}(\check{\boldsymbol{U}}) \rightarrow \check{V}=$ $t_{0}(\check{\boldsymbol{V}})$ is an isomorphism on the classical schemes. These are related to the data of Corollary 2.11 by $\check{\boldsymbol{V}}$ is the derived critical locus $\operatorname{Crit}\left(f: U \rightarrow \mathbb{A}^{1}\right)$, and $\check{V}$ the classical critical locus $T=\operatorname{Crit}(f)$, and $\varphi=\check{\varphi} \circ \check{\imath}^{-1}: T=\check{V} \rightarrow X$.

We have standard fibre sequences on $\check{\boldsymbol{U}}$ :

$$
\begin{aligned}
& \check{\varphi}^{*}\left(\mathbb{L}_{\boldsymbol{X}}\right) \underset{\mathbb{L}_{\check{\varphi}}}{\longrightarrow} \mathbb{L}_{\check{U}} \longrightarrow \mathbb{L}_{\check{U} / \boldsymbol{X}} \longrightarrow \check{\varphi}^{*}\left(\mathbb{L}_{\boldsymbol{X}}\right)[1] \\
& \check{\boldsymbol{\imath}}^{*}\left(\mathbb{L}_{\check{\boldsymbol{V}}}\right) \stackrel{\mathbb{L}_{\check{\boldsymbol{\imath}}}}{\longrightarrow} \mathbb{L}_{\check{U}} \longrightarrow \mathbb{L}_{\check{U} / \check{\boldsymbol{U}}} \longrightarrow \check{\boldsymbol{\imath}}^{*}\left(\mathbb{L}_{\check{\boldsymbol{V}}}\right)[1]
\end{aligned}
$$


Taking determinants gives natural isomorphisms of line bundles on $\check{\boldsymbol{U}}$ :

$$
\begin{aligned}
\operatorname{det} \mathbb{L}_{\check{\boldsymbol{U}}} \cong \check{\varphi}^{*}\left(\operatorname{det} \mathbb{L}_{\boldsymbol{X}}\right) \otimes \operatorname{det} \mathbb{L}_{\check{\boldsymbol{U}} / \boldsymbol{X}}, \\
\operatorname{det} \mathbb{L}_{\check{\boldsymbol{U}}} \cong \check{\boldsymbol{\imath}}^{*}\left(\operatorname{det} \mathbb{L}_{\check{\boldsymbol{V}}}\right) \otimes \operatorname{det} \mathbb{L}_{\check{U} / \check{\boldsymbol{V}}}
\end{aligned}
$$

Equation (2.14) gives $\mathbb{L}_{\check{U} / \check{\boldsymbol{V}}} \simeq \mathbb{T}_{\check{U} / \boldsymbol{X}}$ [2]. So taking determinants we have

$$
\operatorname{det} \mathbb{L}_{\check{U} / \check{V}} \cong \operatorname{det} \mathbb{T}_{\check{U} / \boldsymbol{X}} \cong\left(\operatorname{det} \mathbb{L}_{\check{U} / \boldsymbol{X}}\right)^{*} .
$$

Combining (3.17)-(3.18) and restricting to $\check{U}=t_{0}(\check{\boldsymbol{U}}) \subseteq \check{\boldsymbol{U}}$ yields

$$
\check{\varphi}^{*}\left(\left.\operatorname{det} \mathbb{L}_{\boldsymbol{X}}\right|_{X}\right) \cong \check{\imath}^{*}\left(\left.\operatorname{det} \mathbb{L}_{\check{\boldsymbol{V}}}\right|_{\check{V}}\right) \otimes\left(\left.\operatorname{det} \mathbb{L}_{\check{\boldsymbol{U}} / \boldsymbol{X}}\right|_{\check{U}}\right)^{\otimes^{-2}}
$$

Since $\check{\varphi}: \check{\boldsymbol{U}} \rightarrow \boldsymbol{X}$ is smooth, so is $\check{\varphi}: \check{U} \rightarrow X$, and

$$
\left.\mathbb{L}_{\check{\boldsymbol{U}} / \boldsymbol{X}}\right|_{\check{U}} \cong \mathbb{L}_{\check{U} / X} \cong T_{\breve{U} / X}^{*}
$$

As $\check{i}: \check{U} \rightarrow \check{V}=T$ is an isomorphism, we may apply $\left(\check{i}^{-1}\right)^{*}$ to (3.19). Using (3.20) and $\left(\check{\imath}^{-1}\right)^{*} \circ \check{i}^{*}=\mathrm{id},\left(\check{\imath}^{-1}\right)^{*} \circ \check{\varphi}^{*}=\varphi^{*}$ as $\varphi=\check{\varphi} \circ \check{i}^{-1}$ gives

$$
\varphi^{*}\left(\left.\operatorname{det} \mathbb{L}_{\boldsymbol{X}}\right|_{X}\right) \cong\left(\left.\operatorname{det} \mathbb{L}_{\check{\boldsymbol{V}}}\right|_{T}\right) \otimes\left(\check{i}^{-1}\right)^{*}\left(\Lambda^{\mathrm{top}} T_{\breve{U} / X}^{*}\right)^{\otimes^{-2}}
$$

Since $\check{\boldsymbol{V}}=\operatorname{Crit}\left(f: U \rightarrow \mathbb{A}^{1}\right)$, we have

$$
\left.\mathbb{L}_{\check{V}}\right|_{T} \simeq\left[\left.\left.T U\right|_{T} \stackrel{\left.\partial^{2} f\right|_{T}}{\longrightarrow} T^{*} U\right|_{T}\right],
$$

with $\left.T U\right|_{T}$ in degree -1 and $\left.T^{*} U\right|_{T}$ in degree 0 . Therefore

$$
\left.\operatorname{det} \mathbb{L}_{\check{\boldsymbol{V}}}\right|_{T} \cong i^{*}\left(K_{U}^{\otimes^{2}}\right) .
$$

Also, as $\check{i}^{-1}: T \rightarrow \hat{U}$ is an isomorphism, we have

$$
\left(\check{\imath}^{-1}\right)^{*}\left(T_{U / X}^{*}\right) \cong T_{T / X}^{*} .
$$

Combining (3.21)-(3.23), restricting to $T^{\mathrm{red}}$ and using (3.16) gives

$$
\left.\left(\varphi^{\text {red }}\right)^{*}\left(\left.\operatorname{det} \mathbb{L}_{\boldsymbol{X}}\right|_{X^{\text {red }}}\right) \cong K_{T, s_{T}} \otimes\left(\Lambda^{\text {top }} T_{T / X}^{*}\right)\right|_{T^{\text {red }}} ^{\otimes^{-2}} .
$$

Substituting in the isomorphism $\Gamma_{T, \varphi}$ in Theorem 3.15(b) from the smooth morphism $\varphi: T \rightarrow X$ gives a canonical isomorphism of line bundles on $T^{\mathrm{red}}$ :

$$
\left(\varphi^{\mathrm{red}}\right)^{*}\left(\left.\operatorname{det} \mathbb{L}_{\boldsymbol{X}}\right|_{X^{\mathrm{red}}}\right) \cong K_{X, s}\left(T^{\mathrm{red}}, \varphi^{\mathrm{red}}\right) .
$$

This establishes the isomorphism $\left.K_{X, s} \cong \operatorname{det}\left(\mathbb{L}_{\boldsymbol{X}}\right)\right|_{X^{\text {red }}}$ in Theorem 3.18 (b) evaluated on $\left(T^{\mathrm{red}}, \varphi^{\mathrm{red}}\right)$ for any $U, f, T, \varphi$ coming from Corollary 2.11. Such $\varphi^{\text {red }}: T^{\text {red }} \rightarrow X^{\text {red }}$ form an open cover of $X^{\text {red }}$ in the smooth topology. To prove the isomorphism $\left.K_{X, s} \cong \operatorname{det}\left(\mathbb{L}_{\boldsymbol{X}}\right)\right|_{X^{\text {red }}}$ globally and complete the proof, there are two possible methods. Firstly, we could prove that given two choices $U, f, T, \varphi$ and $U^{\prime}, f^{\prime}, T^{\prime}, \varphi^{\prime}$ in Corollary [2.11, the corresponding isomorphisms (3.25) agree on the overlap $T^{\text {red }} \times_{\varphi^{\text {red }}, X, \varphi^{\prime \text { red }}} T^{\text {red }}$. 
But as we are dealing with line bundles on a reduced stack $X^{\text {red }}$, there is a second, easier way: we can show that for each $t \in T^{\text {red }}$ with $\varphi^{\text {red }}(t)=x \in X^{\text {red }}$, the isomorphism $\left.\left.\operatorname{det} \mathbb{L}_{\boldsymbol{X}}\right|_{x} \cong K_{X, s}\right|_{x}$ from restricting (3.25) to $t$ depends only on $x \in X^{\text {red }}$, and not on the choice of $U, f, T, \varphi, t$. This holds as by Theorem 3.15(a) we have an isomorphism

$$
\left.K_{X, s}\right|_{x} \cong\left(\Lambda^{\text {top }} T_{x}^{*} X\right)^{\otimes^{2}} \otimes\left(\Lambda^{\text {top }} \mathfrak{I s o}_{x}(X)\right)^{\otimes^{2}}
$$

Since $\mathbb{L}_{\boldsymbol{X}}$ is perfect in the interval $[-2,1]$, we have

$$
\left.\operatorname{det} \mathbb{L}_{\boldsymbol{X}}\right|_{x} \cong \bigotimes_{i=-2}^{1}\left(\Lambda^{\text {top }} H^{i}\left(\left.\mathbb{L}_{\boldsymbol{X}}\right|_{x}\right)\right)^{(-1)^{i}},
$$

where we have canonical isomorphisms

$$
\begin{aligned}
H^{0}\left(\left.\mathbb{L}_{\boldsymbol{X}}\right|_{x}\right) & \cong T_{x}^{*} X, \quad H^{1}\left(\left.\mathbb{L}_{\boldsymbol{X}}\right|_{x}\right) \cong \mathfrak{I s o}_{x}(X)^{*} \\
H^{-1}\left(\left.\mathbb{L}_{\boldsymbol{X}}\right|_{x}\right) & \cong T_{x} X, \quad H^{-2}\left(\left.\mathbb{L}_{\boldsymbol{X}}\right|_{x}\right) \cong \mathfrak{I s o}_{x}(X)
\end{aligned}
$$

the first line holding for any derived Artin stack $\boldsymbol{X}$, and the second line from $H^{i}\left(\left.\mathbb{L}_{\boldsymbol{X}}\right|_{x}\right) \cong H^{-1-i}\left(\left.\mathbb{L}_{\boldsymbol{X}}\right|_{x}\right)^{*}$ as $\left(\boldsymbol{X}, \omega_{\boldsymbol{X}}\right)$ is -1-shifted symplectic.

Combining (3.26) -3.28 gives a canonical isomorphism $\left.\left.\operatorname{det} \mathbb{L}_{\boldsymbol{X}}\right|_{x} \cong K_{X, s}\right|_{x}$ depending only on $x \in X^{\text {red }}$. Following through (3.16) (3.25) restricted to $t \in T^{\text {red }}$ with $\varphi^{\text {red }}(t)=x$, we find that the restriction of (3.25) to $t$ gives the same isomorphism. This completes the proof of Theorem 3.18 (b).

\section{Perverse sheaves on d-critical stacks}

In [3, Th. 6.9], given in Theorem 4.4 below, we constructed a natural perverse sheaf $P_{X, s}^{\bullet}$ on an oriented algebraic d-critical locus $(X, s)$. The main result of this section, Theorem 4.8, generalizes this to oriented d-critical stacks.

We begin in 4.1 with some background on perverse sheaves on schemes. Section 4.2 recalls results from [3, and proves in Proposition 4.5] a smooth pullback property of the $P_{X, s}^{\bullet}$ in Theorem 4.4. Section 4.3 discusses perverse sheaves on Artin stacks. Once we have set up all the notation, Theorem 4.8 in $\$ 4.4$ follows almost immediately from Theorem 4.4 and Proposition 4.5. In this section the base field $\mathbb{K}$ may be algebraically closed with char $\mathbb{K} \neq 2$, except in Corollaries 4.9 and 4.10 when we require char $\mathbb{K}=0$ to apply the results of $\$ 3$.

\subsection{Perverse sheaves on schemes}

We will assume the reader is familiar with the theory of perverse sheaves on $\mathbb{C}$ schemes and $\mathbb{K}$-schemes. An introduction to perverse sheaves on schemes suited to our purposes can be found in [3, §2], and our definitions and notation follow that paper. Here is a brief survival guide:

- We work throughout this section over an algebraically closed field $\mathbb{K}$ with char $\mathbb{K} \neq 2$, for instance $\mathbb{K}=\mathbb{C}$. All $\mathbb{K}$-schemes $X, Y, Z, \ldots$ are assumed separated and of finite type. 
- We work with constructible complexes and perverse sheaves over a commutative base ring $A$. The allowed rings $A$ depend on the field $\mathbb{K}$. For $\mathbb{K}=\mathbb{C}$ one can define perverse sheaves using the complex analytic topology as in Dimca [7, and then $A$ can be essentially arbitrary, e.g. $A=\mathbb{Q}$ or $\mathbb{Z}$.

If $\mathbb{K} \neq \mathbb{C}$ then one must define perverse sheaves using the étale topology, as in Beilinson, Bernstein and Deligne 11. Then the allowed possibilities are $A$ with char $A>0$ coprime to char $\mathbb{K}$, or the $l$-adic integers $\mathbb{Z}_{l}$, or the $l$-adic rationals $\mathbb{Q}_{l}$, or its algebraic closure $\overline{\mathbb{Q}}_{l}$, for $l$ a prime coprime to char $\mathbb{K}$. We will refer to all these possibilities as l-adic perverse sheaves.

- For a $\mathbb{K}$-scheme $X$, one defines the derived category $D_{c}^{b}(X)$ of constructible complexes of $A$-modules on $X$. There is a natural t-structure on $D_{c}^{b}(X)$, with heart the abelian category $\operatorname{Perv}(X)$ of perverse sheaves on $X$.

- An example of a constructible complex on $X$ is the constant sheaf $A_{X}$ with fibre $A$ at each point. If $X$ is smooth then $A_{X}[\operatorname{dim} X] \in \operatorname{Perv}(X)$.

- Grothendieck's "six operations on sheaves" $f^{*}, f^{!}, R f_{*}, R f_{!}, \mathcal{R H o m}, \stackrel{L}{\otimes}$ act on the categories $D_{c}^{b}(X)$. There is a functor $\mathbb{D}_{X}: D_{c}^{b}(X) \rightarrow D_{c}^{b}(X)^{\text {op }}$ with $\mathbb{D}_{X} \circ \mathbb{D}_{X} \cong \mathrm{id}: D_{c}^{b}(X) \rightarrow D_{c}^{b}(X)$, called Verdier duality.

- Let $U$ be a $\mathbb{K}$-scheme and $f: U \rightarrow \mathbb{A}^{1}$ a regular function, and write $U_{0}$ for the subscheme $f^{-1}(0) \subseteq U$. Then one can define the nearby cycle functor $\psi_{f}^{p}: D_{c}^{b}(U) \rightarrow D_{c}^{b}\left(U_{0}\right)$ and the vanishing cycle functor $\phi_{f}^{p}: D_{c}^{b}(U) \rightarrow$ $D_{c}^{b}\left(U_{0}\right)$. Both map $\operatorname{Perv}(U) \rightarrow \operatorname{Perv}\left(U_{0}\right)$.

- Let $U$ be a smooth $\mathbb{K}$-scheme and $f: U \rightarrow \mathbb{A}^{1}$ a regular function, and write $X=\operatorname{Crit}(f)$. Then we have a decomposition $X=\coprod_{c \in f(X)} X_{c}$, where $X_{c} \subseteq X$ is the open and closed subscheme of points $p \in X$ with $f(p)=c$. It turns out that $\phi_{f}^{p}\left(A_{U}[\operatorname{dim} U]\right)$ is supported on $X_{0} \subseteq X \subseteq U$.

Following [3, §2.4], define the perverse sheaf of vanishing cycles $\mathcal{P} \mathcal{V}_{U, f}^{\bullet}$ of $U, f$ in $\operatorname{Perv}(X)$ or $\operatorname{Perv}(U)$ to be $\mathcal{P} \mathcal{V}_{U, f}^{\bullet}=\left.\bigoplus_{c \in f(X)} \phi_{f-c}^{p}\left(A_{U}[\operatorname{dim} U]\right)\right|_{X_{c}}$. We also define a canonical Verdier duality isomorphism

$$
\sigma_{U, f}: \mathcal{P} \mathcal{V}_{U, f}^{\bullet} \stackrel{\cong}{\longrightarrow} \mathbb{D}_{X}\left(\mathcal{P} \mathcal{V}_{U, f}^{\bullet}\right)
$$

and twisted monodromy operator

$$
\tau_{U, f}: \mathcal{P} \mathcal{V}_{U, f}^{\bullet} \stackrel{\cong}{\longrightarrow} \mathcal{V}_{U, f}^{\bullet} .
$$

Some references are [3, §2], Dimca [7] for perverse sheaves on $\mathbb{C}$-schemes, and Beilinson, Bernstein and Deligne [1, Ekedahl [8], Freitag and Kiehl [9], and Kiehl and Weissauer [17 for perverse sheaves on $\mathbb{K}$-schemes.

The theories of $\mathscr{D}$-modules on $\mathbb{K}$-schemes, and Saito's mixed Hodge modules on $\mathbb{C}$-schemes, also share this whole package of properties, and our results also generalize to $\mathscr{D}$-modules and mixed Hodge modules, as in 3 .

Here are some results connecting perverse sheaves and smooth morphisms. Theorem [4.2 (proved in [1, Th. 3.2.4], see also [22, §2.3]) is the reason why perverse sheaves extend to Artin stacks, as we discuss in $\$ 4.3$. 
Proposition 4.1. Let $\Phi: X \rightarrow Y$ be a scheme morphism smooth of relative dimension $d$. Then the (exceptional) inverse image functors $\Phi^{*}, \Phi^{!}: D_{c}^{b}(Y) \rightarrow$ $D_{c}^{b}(X)$ satisfy $\Phi^{*}[d] \cong \Phi^{!}[-d]$, where $\Phi^{*}[d], \Phi^{!}[-d]$ are $\Phi^{*}, \Phi^{!}$shifted by $\pm d$. Furthermore $\Phi^{*}[d], \Phi^{!}[-d]$ map $\operatorname{Perv}(Y) \rightarrow \operatorname{Perv}(X)$.

Theorem 4.2. Let $X$ be a scheme. Then perverse sheaves on $X$ form a stack (a kind of sheaf of categories) on $X$ in the smooth topology.

Explicitly, this means the following. Let $\left\{u_{i}: U_{i} \rightarrow X\right\}_{i \in I}$ be a smooth open cover for $X$, so that $u_{i}: U_{i} \rightarrow X$ is a scheme morphism smooth of relative dimension $d_{i}$ for $i \in I$, with $\coprod_{i} u_{i}$ surjective. Write $U_{i j}=U_{i} \times_{u_{i}, X, u_{j}} U_{j}$ for $i, j \in I$ with projections

$$
\pi_{i j}^{i}: U_{i j} \longrightarrow U_{i}, \quad \pi_{i j}^{j}: U_{i j} \longrightarrow U_{j}, \quad u_{i j}=u_{i} \circ \pi_{i j}^{i}=u_{j} \circ \pi_{i j}^{j}: U_{i j} \longrightarrow X .
$$

Similarly, write $U_{i j k}=U_{i} \times{ }_{X} U_{j} \times{ }_{X} U_{k}$ for $i, j, k \in I$ with projections

$$
\begin{gathered}
\pi_{i j k}^{i j}: U_{i j k} \longrightarrow U_{i j}, \quad \pi_{i j k}^{i k}: U_{i j k} \longrightarrow U_{i k}, \quad \pi_{i j k}^{j k}: U_{i j k} \longrightarrow U_{j k}, \\
\pi_{i j k}^{i}: U_{i j k} \rightarrow U_{i}, \pi_{i j k}^{j}: U_{i j k} \rightarrow U_{j}, \pi_{i j k}^{k}: U_{i j k} \rightarrow U_{k}, u_{i j k}: U_{i j k} \rightarrow X,
\end{gathered}
$$

so that $\pi_{i j k}^{i}=\pi_{i j}^{i} \circ \pi_{i j k}^{i j}, u_{i j k}=u_{i j} \circ \pi_{i j k}^{i j}=u_{i} \circ \pi_{i j k}^{i}$, and so on. All these morphisms $u_{i}, \pi_{i j}^{i}, \ldots, u_{i j k}$ are smooth of known relative dimensions, so $u_{i}^{*}\left[d_{i}\right] \cong u_{i}^{!}\left[-d_{i}\right]$ maps $\operatorname{Perv}(X) \rightarrow \operatorname{Perv}\left(U_{i}\right)$ by Proposition 4.1, and similarly for $\pi_{i j}^{i}, \ldots, u_{i j k}$. With this notation:

(i) Suppose $\mathcal{P}^{\bullet}, \mathcal{Q}^{\bullet} \in \operatorname{Perv}(X)$, and we are given $\alpha_{i}: u_{i}^{*}\left[d_{i}\right]\left(\mathcal{P}^{\bullet}\right) \rightarrow u_{i}^{*}\left[d_{i}\right]\left(\mathcal{Q}^{\bullet}\right)$ in $\operatorname{Perv}\left(U_{i}\right)$ for all $i \in I$ such that for all $i, j \in I$ we have

$$
\left(\pi_{i j}^{i}\right)^{*}\left[d_{j}\right]\left(\alpha_{i}\right)=\left(\pi_{i j}^{j}\right)^{*}\left[d_{i}\right]\left(\alpha_{j}\right): u_{i j}^{*}\left[d_{i}+d_{j}\right]\left(\mathcal{P}^{\bullet}\right) \longrightarrow u_{i j}^{*}\left[d_{i}+d_{j}\right]\left(\mathcal{Q}^{\bullet}\right) .
$$

Then there is a unique $\alpha: \mathcal{P}^{\bullet} \rightarrow \mathcal{Q}^{\bullet}$ with $\alpha_{i}=u_{i}^{*}\left[d_{i}\right](\alpha)$ for all $i \in I$.

(ii) Suppose we are given $\mathcal{P}_{i}^{\bullet} \in \operatorname{Perv}\left(U_{i}\right)$ for all $i \in I$ and isomorphisms $\alpha_{i j}$ : $\left(\pi_{i j}^{i}\right)^{*}\left[d_{j}\right]\left(\mathcal{P}_{i}^{\bullet}\right) \rightarrow\left(\pi_{i j}^{j}\right)^{*}\left[d_{i}\right]\left(\mathcal{P}_{j}^{\bullet}\right)$ in $\operatorname{Perv}\left(U_{i j}\right)$ for all $i, j \in I$ with $\alpha_{i i}=\mathrm{id}$ and

$$
\begin{gathered}
\left(\pi_{i j k}^{j k}\right)^{*}\left[d_{i}\right]\left(\alpha_{j k}\right) \circ\left(\pi_{i j k}^{i j}\right)^{*}\left[d_{k}\right]\left(\alpha_{i j}\right)=\left(\pi_{i j k}^{i k}\right)^{*}\left[d_{j}\right]\left(\alpha_{i k}\right):\left(\pi_{i j k}^{i}\right)^{*}\left[d_{j}+d_{k}\right]\left(\mathcal{P}_{i}\right) \\
\longrightarrow\left(\pi_{i j k}^{k}\right)^{*}\left[d_{i}+d_{j}\right]\left(\mathcal{P}_{k}\right)
\end{gathered}
$$

in $\operatorname{Perv}\left(U_{i j k}\right)$ for all $i, j, k \in I$. Then there exists $\mathcal{P}^{\bullet}$ in $\operatorname{Perv}(X)$, unique up to canonical isomorphism, with isomorphisms $\beta_{i}: u_{i}^{*}\left(\mathcal{P}^{\bullet}\right) \rightarrow \mathcal{P}_{i}^{\bullet}$ for each $i \in I$, satisfying $\alpha_{i j} \circ\left(\pi_{i j}^{i}\right)^{*}\left(\beta_{i}\right)=\left(\pi_{i j}^{j}\right)^{*}\left(\beta_{j}\right): u_{i j}^{*}\left(\mathcal{P}^{\bullet}\right) \rightarrow\left(\pi_{i j}^{j}\right)^{*}\left(\mathcal{P}_{j}^{\bullet}\right)$ for all $i, j \in I$.

Proposition 4.3. Let $\Phi: U \rightarrow V$ be a scheme morphism smooth of relative dimension $d$ and $g: V \rightarrow \mathbb{A}^{1}$ be regular, and set $f=g \circ \Phi: U \rightarrow \mathbb{A}^{1}$. Then

(a) There are natural isomorphisms of functors $\operatorname{Perv}(V) \rightarrow \operatorname{Perv}\left(U_{0}\right)$ :

$$
\Phi_{0}^{*}[d] \circ \psi_{g}^{p} \cong \psi_{f}^{p} \circ \Phi^{*}[d] \quad \text { and } \quad \Phi_{0}^{*}[d] \circ \phi_{g}^{p} \cong \phi_{f}^{p} \circ \Phi^{*}[d],
$$

where $U_{0}=f^{-1}(0) \subseteq U, V_{0}=g^{-1}(0) \subseteq V$ and $\Phi_{0}=\left.\Phi\right|_{U_{0}}: U_{0} \rightarrow V_{0}$. 
(b) Write $X=\operatorname{Crit}(f)$ and $Y=\operatorname{Crit}(g)$, so that $\left.\Phi\right|_{X}: X \rightarrow Y$ is smooth of dimension $d$. Then there is a canonical isomorphism

$$
\Xi_{\Phi}:\left.\Phi\right|_{X} ^{*}[d]\left(\mathcal{P} \mathcal{V}_{V, g}^{\bullet} \stackrel{\cong}{\longrightarrow} \mathcal{P} \mathcal{V}_{U, f}^{\bullet} \quad \text { in } \operatorname{Perv}(X)\right.
$$

which identifies $\left.\Phi\right|_{X} ^{*}[d]\left(\sigma_{V, g}\right),\left.\Phi\right|_{X} ^{*}[d]\left(\tau_{V, g}\right)$ with $\sigma_{U, f}, \tau_{U, f}$.

\subsection{Perverse sheaves on d-critical loci}

Here is [3, Th. 6.9], which we will generalize to stacks in Theorem 4.8 below. We use the notation of $\$ 3.1$ and $\$ 4.1$ throughout.

Theorem 4.4. Let $(X, s)$ be an oriented algebraic d-critical locus over $\mathbb{C}$, with orientation $K_{X, s}^{1 / 2}$. Then for any well-behaved base ring $A$, such as $\mathbb{Z}, \mathbb{Q}$ or $\mathbb{C}$, there exists a perverse sheaf $P_{X, s}^{\bullet}$ in $\operatorname{Perv}(X)$ over $A$, which is natural up to canonical isomorphism, and Verdier duality and monodromy isomorphisms

$$
\Sigma_{X, s}: P_{X, s}^{\bullet} \longrightarrow \mathbb{D}_{X}\left(P_{X, s}^{\bullet}\right), \quad \mathrm{T}_{X, s}: P_{X, s}^{\bullet} \longrightarrow P_{X, s}^{\bullet},
$$

which are characterized by the following properties:

(i) If $(R, U, f, i)$ is a critical chart on $(X, s)$, there is a natural isomorphism

$$
\omega_{R, U, f, i}:\left.P_{X, s}^{\bullet}\right|_{R} \longrightarrow i^{*}\left(\mathcal{P} \mathcal{V}_{U, f}^{\bullet}\right) \otimes_{\mathbb{Z} / 2 \mathbb{Z}} Q_{R, U, f, i},
$$

where $\pi_{R, U, f, i}: Q_{R, U, f, i} \rightarrow R$ is the principal $\mathbb{Z} / 2 \mathbb{Z}$-bundle parametrizing local isomorphisms $\alpha:\left.K_{X, s}^{1 / 2} \rightarrow i^{*}\left(K_{U}\right)\right|_{R^{\mathrm{red}}}$ with $\alpha \otimes \alpha=\iota_{R, U, f, i}$, for $\iota_{R, U, f, i}$ as in (3.4). Furthermore the following commute in $\operatorname{Perv}(R)$ :

$$
\begin{aligned}
& \left.P_{X, s}^{\bullet}\right|_{R} \longrightarrow i^{*}\left(\mathcal{P} \mathcal{V}_{U, f}^{\bullet}\right) \otimes_{\mathbb{Z} / 2 \mathbb{Z}} Q_{R, U, f, i} \\
& \begin{array}{ll}
\left.\sum_{X, s}\right|_{R} & i^{*}\left(\sigma_{U, f}\right) \otimes \operatorname{id}_{Q_{R, U, f, i}} \downarrow \\
\mathbb{D}_{R}\left(\left.P_{X, s}^{\bullet}\right|_{R}\right) \longleftarrow \mathbb{D}_{R}\left(\omega_{R, U, f, i}\right) & i^{*}\left(\mathbb{D}_{\operatorname{Crit}(f)}\left(\mathcal{P} \mathcal{V}_{U, f}^{\bullet}\right)\right) \otimes_{\mathbb{Z} / 2 \mathbb{Z}} Q_{R, U, f, i} \\
\cong \mathbb{D}_{R}\left(i^{*}\left(\mathcal{P} \mathcal{V}_{U, f}^{\bullet}\right) \otimes_{\mathbb{Z} / 2 \mathbb{Z}} Q_{R, U, f, i}\right),
\end{array} \\
& \left.P_{X, s}^{\bullet}\right|_{R} \longrightarrow i^{*}\left(\mathcal{P} \mathcal{V}_{U, f}^{\bullet}\right) \otimes_{\mathbb{Z} / 2 \mathbb{Z}} Q_{R, U, f, i} \\
& \left.\downarrow \mathrm{T}_{X, s}\right|_{R} \quad \omega_{R, U, f, i} \quad i^{*}\left(\tau_{U, f}\right) \otimes \operatorname{id}_{Q_{R, U, f, i}} \downarrow \\
& \left.P_{X, s}^{\bullet}\right|_{R} \omega_{R, U, f, i} i^{*}\left(\mathcal{P} \mathcal{V}_{U, f}^{\bullet}\right) \otimes_{\mathbb{Z} / 2 \mathbb{Z}} Q_{R, U, f, i} \text {. }
\end{aligned}
$$

(ii) If $\Phi:(R, U, f, i) \hookrightarrow(S, V, g, j)$ is an embedding of critical charts on $(X, s)$, there is a compatibility condition [3, Th. 6.9(ii)] between $\omega_{R, U, f, i}, \omega_{S, V, g, j}$ which we will not give.

Analogues hold for oriented algebraic d-critical loci $(X, s)$ over general fields $\mathbb{K}$ in the settings of l-adic perverse sheaves and of $\mathscr{D}$-modules, and for oriented algebraic d-critical loci $(X, s)$ over $\mathbb{C}$ in the setting of mixed Hodge modules. 
We prove a proposition on the behaviour of the perverse sheaves $P_{X, s}^{\bullet}$ of Theorem 4.4 under smooth pullback, which will be the main ingredient in the proof of our main result Theorem 4.8 .

Proposition 4.5. (a) Let $\phi:(X, s) \rightarrow(Y, t)$ be a morphism of algebraic $d$ critical loci over $\mathbb{C}$, in the sense of 3.1 and suppose $\phi: X \rightarrow Y$ is smooth of relative dimension d. Let $K_{Y, t}^{1 / 2}$ be an orientation for $(Y, t)$, so that Corollary 3.8 defines an induced orientation $K_{X, s}^{1 / 2}$ for $(X, s)$. Theorem 4.4 defines perverse sheaves $P_{X, s}^{\bullet}, P_{Y, t}^{\bullet}$ on $X, Y$. Then there is a natural isomorphism

$$
\Delta_{\phi}: \phi^{*}[d]\left(P_{Y, t}^{\bullet} \stackrel{\cong}{\longrightarrow} P_{X, s}^{\bullet} \quad \text { in } \operatorname{Perv}(X)\right.
$$

which is characterized by the property that if $(R, U, f, i),(S, V, g, j)$ are critical charts on $(X, s),(Y, t)$ with $\phi(R) \subseteq S$ and $\Phi: U \rightarrow V$ is smooth of relative dimension $d$ with $f=g \circ \Phi$ and $\Phi \circ i=j \circ \phi$, then the following commutes

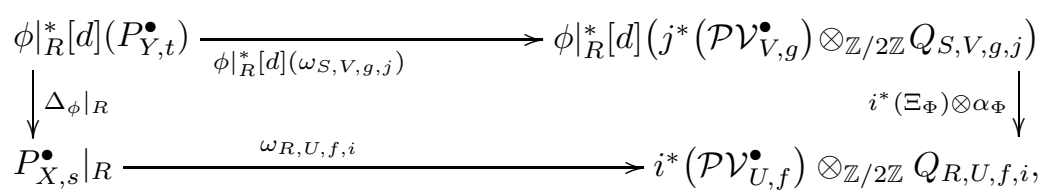

where $\Xi_{\Phi}$ is as in (4.2) and $\alpha_{\Phi}:\left.\phi\right|_{R} ^{*}[d]\left(Q_{S, V, g, j}\right) \rightarrow Q_{R, U, f, i}$ is the natural isomorphism. Also $\Delta_{\phi}$ identifies $\phi^{*}[d]\left(\Sigma_{Y, t}\right), \phi^{*}[d]\left(\mathrm{T}_{Y, t}\right)$ with $\Sigma_{X, s}, \mathrm{~T}_{X, s}$.

(b) If $\psi:(Y, t) \rightarrow(Z, u)$ is another morphism of algebraic d-critical loci over $\mathbb{C}$ smooth of relative dimension $e$, then

$$
\Delta_{\psi \circ \phi}=\Delta_{\phi} \circ \phi^{*}[d]\left(\Delta_{\psi}\right):(\psi \circ \phi)^{*}[d+e]\left(P_{Z, u}^{\bullet}\right) \stackrel{\cong}{\longrightarrow} P_{X, s}^{\bullet} .
$$

(c) Analogues of (a),(b) hold for algebraic d-critical loci $(X, s)$ over general fields $\mathbb{K}$ in the settings of l-adic perverse sheaves and of $\mathscr{D}$-modules, and for algebraic d-critical loci $(X, s)$ over $\mathbb{C}$ in the setting of mixed Hodge modules.

Proof. Let $\phi:(X, s) \rightarrow(Y, t), d, K_{Y, t}^{1 / 2}, K_{X, s}^{1 / 2}, P_{X, s}^{\bullet}, P_{Y, t}^{\bullet}$ be as in (a). If $x \in X$ with $\phi(x)=y \in Y$ then the proof of [15, Prop. 2.8] shows that we may choose critical charts $(R, U, f, i),(S, V, g, j)$ on $(X, s),(Y, t)$ with $x \in R, y \in \phi(R) \subseteq S$ of minimal dimensions $\operatorname{dim} U=\operatorname{dim} T_{x} X, \operatorname{dim} V=\operatorname{dim} T_{y} Y$, and $\Phi: U \rightarrow V$ smooth of relative dimension $d$ with $f=g \circ \Phi$ and $\Phi \circ i=j \circ \phi$.

Choose such data $\left(R_{a}, U_{a}, f_{a}, i_{a}\right),\left(S_{a}, V_{a}, g_{a}, j_{a}\right), \Phi_{a}$ for $a \in A$, an indexing set, such that $\left\{R_{a}: a \in A\right\}$ is an open cover for $X$. For each $a \in A$, define an isomorphism $\Delta_{a}:\left.\left.\phi\right|_{R_{a}} ^{*}[d]\left(P_{Y, t}^{\bullet}\right) \rightarrow P_{X, s}^{\bullet}\right|_{R_{a}}$ to make the following diagram of isomorphisms commute, the analogue of (4.6):

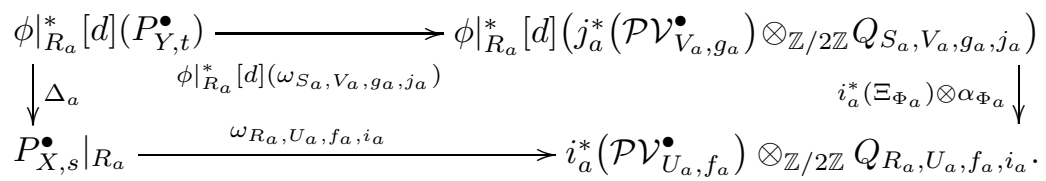


Combining the last part of Proposition 4.3(b) with (4.3)-(4.4) shows that this $\Delta_{a}$ identifies $\left.\phi^{*}[d]\left(\Sigma_{Y, t}\right)\right|_{R_{a}},\left.\phi^{*}[d]\left(\mathrm{T}_{Y, t}\right)\right|_{R_{a}}$ with $\left.\Sigma_{X, s}\right|_{R_{a}},\left.\mathrm{~T}_{X, s}\right|_{R_{a}}$.

We claim that for all $a, b \in A$ we have $\left.\Delta_{a}\right|_{R_{a} \cap R_{b}}=\left.\Delta_{b}\right|_{R_{a} \cap R_{b}}$. To prove this, let $x \in R_{a} \cap R_{b}$, with $y=f(x) \in S_{a} \cap S_{b}$. By Theorem 3.3 we can choose subcharts $\left(R_{a}^{\prime}, U_{a}^{\prime}, f_{a}^{\prime}, i_{a}^{\prime}\right) \subseteq\left(R_{a}, U_{a}, f_{a}, i_{a}\right),\left(R_{b}^{\prime}, U_{b}^{\prime}, f_{b}^{\prime}, i_{b}^{\prime}\right) \subseteq\left(R_{b}, U_{b}, f_{b}, i_{b}\right)$, $\left(S_{a}^{\prime}, V_{a}^{\prime}, g_{a}^{\prime}, j_{a}^{\prime}\right) \subseteq\left(S_{a}, V_{a}, g_{a}, j_{a}\right),\left(S_{b}^{\prime}, V_{b}^{\prime}, g_{b}^{\prime}, j_{b}^{\prime}\right) \subseteq\left(S_{b}, V_{b}, g_{b}, j_{b}\right)$ with $x \in R_{a}^{\prime} \cap$ $R_{b}^{\prime}, y \in S_{a}^{\prime} \cap S_{b}^{\prime}$, critical charts $\left(R_{a b}, U_{a b}, f_{a b}, i_{a b}\right),\left(S_{a b}, V_{a b}, g_{a b}, j_{a b}\right)$ on $(X, s)$, $(Y, t)$, and embeddings $\Psi_{a}:\left(R_{a}^{\prime}, U_{a}^{\prime}, f_{a}^{\prime}, i_{a}^{\prime}\right) \hookrightarrow\left(R_{a b}, U_{a b}, f_{a b}, i_{a b}\right), \Psi_{b}:\left(R_{b}^{\prime}, U_{b}^{\prime}\right.$, $\left.f_{b}^{\prime}, i_{b}^{\prime}\right) \hookrightarrow\left(R_{a b}, U_{a b}, f_{a b}, i_{a b}\right), \Omega_{a}:\left(S_{a}^{\prime}, V_{a}^{\prime}, g_{a}^{\prime}, j_{a}^{\prime}\right) \hookrightarrow\left(S_{a b}, V_{a b}, g_{a b}, j_{a b}\right)$, and $\Omega_{b}$ : $\left(S_{b}^{\prime}, V_{b}^{\prime}, g_{b}^{\prime}, j_{b}^{\prime}\right) \hookrightarrow\left(S_{a b}, V_{a b}, g_{a b}, j_{a b}\right)$.

By combining the proofs of Proposition 3.2 and Theorem 3.3 in [15], we can show that we can choose this data such that $\Phi_{a}\left(U_{a}^{\prime}\right) \subseteq V_{a}^{\prime}, \Phi_{b}\left(U_{b}^{\prime}\right) \subseteq V_{b}^{\prime}$, and with a morphism $\Phi_{a b}: U_{a b} \rightarrow V_{a b}$ smooth of relative dimension $d$ such that

$$
\begin{aligned}
f_{a b} & =g_{a b} \circ \Phi_{a b}, & & \Phi_{a b} \circ i_{a b}=j_{a b} \circ \phi_{a b}, \\
\Phi_{a b} \circ \Psi_{a} & =\left.\Omega_{a} \circ \Phi_{a}\right|_{U_{a}^{\prime}}, & & \Phi_{a b} \circ \Psi_{b}=\left.\Omega_{b} \circ \Phi_{a}\right|_{U_{a}^{\prime}} .
\end{aligned}
$$

As for (4.8) we have a commutative diagram

$$
\begin{aligned}
& \left.\left.\phi\right|_{R_{a b}} ^{*}[d]\left(P_{Y, t}^{\bullet}\right) \longrightarrow \phi\right|_{R_{a b}} ^{*}[d]\left(j_{a b}^{*}\left(\mathcal{P} \mathcal{V}_{V_{a b}, g_{a b}}^{\bullet}\right) \otimes_{\mathbb{Z} / 2 \mathbb{Z}} Q_{S_{a b}, V_{a b}, g_{a b}, j_{a b}}\right) \\
& \left.\downarrow \Delta_{a b} \quad \phi\right|_{R_{a b}} ^{*}[d]\left(\omega_{S_{a b}, V_{a b}, g_{a b}, j_{a b}}\right) \quad i_{a b}^{*}\left(\Xi_{\Phi_{a b}}\right) \otimes \alpha_{\Phi_{a b}} \downarrow \\
& \left.P_{X, s}^{\bullet}\right|_{R_{a b}} \stackrel{\omega_{R_{a b}, U_{a b}, f_{a b}, i a b}}{\longrightarrow} i_{a b}^{*}\left(\mathcal{P} \mathcal{V}_{U_{a b}, f_{a b}}^{\bullet}\right) \otimes_{\mathbb{Z} / 2 \mathbb{Z}} Q_{R_{a b}, U_{a b}, f_{a b}, i_{a b}} .
\end{aligned}
$$

Using [3, Th. 6.9(ii)] for the embeddings $\Psi_{a}, \Omega_{a}$ gives commutative diagrams

$$
\begin{aligned}
& \left.P_{X, s}^{\bullet}\right|_{R_{a}^{\prime}} \longrightarrow i_{a}^{\prime *}\left(\mathcal{P} \mathcal{V}_{U_{a}^{\prime}, f_{a}^{\prime}, U_{a}^{\prime}, f_{a}^{\prime}, i_{a}^{\prime}}\right) \otimes_{\mathbb{Z} / 2 \mathbb{Z}} Q_{R_{a}^{\prime}, U_{a}^{\prime}, f_{a}^{\prime}, i_{a}^{\prime}} \\
& \downarrow \omega_{R_{a b}, U_{a b}, f_{a b},\left.i_{a b}\right|_{R_{a}^{\prime}}} i_{a}^{\prime *}\left(\Theta_{\Psi_{a}}\right) \otimes \mathrm{id} \downarrow \\
& \left.i_{a b}^{*}\left(\mathcal{P} \mathcal{V}_{U_{a b}, f_{a b}}^{\bullet}\right)\right|_{R_{a}^{\prime}} \quad \mathrm{id} \otimes \Lambda_{\Psi_{a}} \longrightarrow i_{a}^{\prime *}\left(\Psi_{a}^{*}\left(\mathcal{P} \mathcal{V}_{U_{a b}, f_{a b}}^{\bullet}\right) \otimes_{\mathbb{Z} / 2 \mathbb{Z}} P_{\Psi_{a}}\right) \\
& \left.\otimes_{\mathbb{Z} / 2 \mathbb{Z}} Q_{R_{a b}, U_{a b}, f_{a b}, i_{a b}}\right|_{R_{a}^{\prime}} \longrightarrow \otimes_{\mathbb{Z} / 2 \mathbb{Z}} Q_{R_{a}^{\prime}, U_{a}^{\prime}, f_{a}^{\prime}, i_{a}^{\prime}}, \\
& \left.P_{Y, t}^{\bullet}\right|_{S_{a}^{\prime}} \underset{\omega_{S_{a}^{\prime}, V_{a}^{\prime}, g_{a}^{\prime}, i_{a}^{\prime}}}{\longrightarrow} j_{a}^{\prime *}\left(\mathcal{P} \mathcal{V}_{V_{a}^{\prime}, g_{a}^{\prime}}^{\bullet}\right) \otimes_{\mathbb{Z} / 2 \mathbb{Z}} Q_{S_{a}^{\prime}, V_{a}^{\prime}, g_{a}^{\prime}, j_{a}^{\prime}} \\
& \left|\omega_{S_{a b}, V_{a b}, g_{a b}, j_{a b} \mid}\right|_{S_{a}^{\prime}} \quad j_{a}^{*}\left(\Theta_{\Omega_{a}}\right) \otimes \mathrm{id} \mid \\
& \begin{array}{ll}
\left.j_{a b}^{*}\left(\mathcal{P} \mathcal{V}_{V_{a b}, g_{a b}}^{\bullet}\right)\right|_{S_{a}^{\prime}} \quad \operatorname{id} \otimes \Lambda_{\Omega_{a}} & j_{a}^{\prime *}\left(\Omega_{a}^{*}\left(\mathcal{P} \mathcal{V}_{V_{a b}, g_{a b}}^{\bullet}\right) \otimes_{\mathbb{Z} / 2 \mathbb{Z}} P_{\Omega_{a}}\right) \\
\left.\otimes_{\mathbb{Z} / 2 \mathbb{Z}} Q_{S_{a b}, V_{a b}, g_{a b}, j_{a b}}\right|_{S_{a}^{\prime}} & { }_{\otimes_{\mathbb{Z} / 2 \mathbb{Z}} Q_{S_{a}^{\prime}, V_{a}^{\prime}, g_{a}^{\prime}, j_{a}^{\prime}} .}
\end{array}
\end{aligned}
$$

Here $P_{\Psi_{a}}, P_{\Omega_{a}}$ are principal $\mathbb{Z} / 2 \mathbb{Z}$-bundles on $R_{a}^{\prime}, S_{a}^{\prime}$ from [3, Def. 5.2], and $\Theta_{\Psi_{a}}, \Theta_{\Omega_{a}}$ are isomorphisms of perverse sheaves from [3, Th. 5.4(a)], and $\Lambda_{\Psi_{a}}$, $\Lambda_{\Omega_{a}}$ are isomorphisms of principal $\mathbb{Z} / 2 \mathbb{Z}$-bundles from [3, Th. 6.9(ii)].

From the definitions of $P_{\Psi_{a}}, P_{\Omega_{a}}, \Theta_{\Psi_{a}}, \Theta_{\Omega_{a}}, \Lambda_{\Psi_{a}}, \Lambda_{\Omega_{a}}$ one can show that there is a natural isomorphism $\beta_{a}: \Phi_{a}^{*}[d]\left(P_{\Omega_{a}}\right) \rightarrow P_{\Psi_{a}}$ such that the follow- 
ing commute:

$$
\begin{aligned}
& \begin{array}{ll}
\Phi_{a}^{*}[d]\left(\mathcal{P} \mathcal{V}_{V_{a}^{\prime}, g_{a}^{\prime}}^{\bullet}\right) & \Phi_{a}^{*}[d]\left(\Omega_{a}^{*}\left(\mathcal{P} \mathcal{V}_{V_{a b}, g_{a b}}^{\bullet}\right) \otimes_{\mathbb{Z} / 2 \mathbb{Z}} P_{\Omega_{a}}\right)= \\
^{*}[d]\left(\Theta_{\Omega_{a}}\right)
\end{array} & \begin{array}{c}
\Psi_{a}^{*} \circ \Phi_{a b}^{*}[d]\left(\mathcal{P} \mathcal{V}_{V_{a b}, g_{a b}}^{\bullet}\right) \otimes_{\mathbb{Z} / 2 \mathbb{Z}} \Phi_{a}^{*}[d]\left(P_{\Omega_{a}}\right) \\
\Xi_{\Phi_{a}} \mathcal{V}_{U_{a}^{\prime}, f_{a}^{\prime}}^{\bullet} \\
\Theta_{\Psi_{a}}
\end{array} \\
& \Psi_{a}^{*}\left(\Xi_{\Phi_{a b}}\right) \otimes \beta_{a} \downarrow \\
& \Psi_{a}^{*}\left(\mathcal{P} \mathcal{V}_{U_{a b}, f_{a b}}^{\bullet}\right) \otimes_{\mathbb{Z} / 2 \mathbb{Z}} P_{\Psi_{a}},
\end{aligned} } \\
{\left.\left.\phi\right|_{R_{a b}} ^{*}[d]\left(Q_{S_{a b}, V_{a b}, g_{a b}, j_{a b}}\right) \longrightarrow \phi\right|_{R_{a b}} ^{*}[d]\left(j_{a}^{\prime *}\left(P_{\Omega_{a}}\right) \otimes_{\mathbb{Z} / 2 \mathbb{Z}} Q_{S_{a}^{\prime}, V_{a}^{\prime}, g_{a}^{\prime}, j_{a}^{\prime}}\right)}
\end{array}
$$

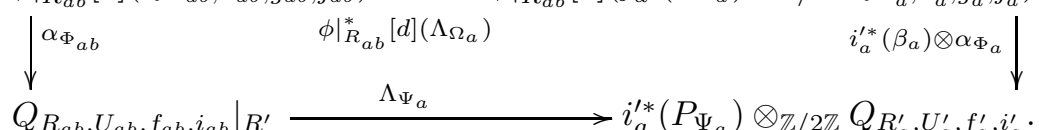

Combining (4.8)- (4.13) we see that $\left.\Delta_{a}\right|_{R_{a}^{\prime}}=\left.\Delta_{a b}\right|_{R_{a}^{\prime}}$. Similarly $\left.\Delta_{b}\right|_{R_{b}^{\prime}}=\left.\Delta_{a b}\right|_{R_{b}^{\prime}}$, so $\left.\Delta_{a}\right|_{R_{a}^{\prime} \cap R_{b}^{\prime}}=\left.\Delta_{b}\right|_{R_{a}^{\prime} \cap R_{b}^{\prime}}$, where $R_{a}^{\prime} \cap R_{b}^{\prime}$ is an open neighbourhood of $x$ in $R_{a} \cap R_{b}$. As we can cover $R_{a} \cap R_{b}$ by such open $R_{a}^{\prime} \cap R_{b}^{\prime}$, and (iso)morphisms of perverse sheaves form a sheaf, it follows that $\left.\Delta_{a}\right|_{R_{a} \cap R_{b}}=\left.\Delta_{b}\right|_{R_{a} \cap R_{b}}$.

By the Zariski topology version of Theorem 4.2(i), there exists a unique isomorphism $\Delta_{\phi}$ in (4.5) such that $\left.\Delta_{\phi}\right|_{R_{a}}=\Delta_{a}$ for all $a \in A$. As each $\Delta_{a}$ identifies $\left.\phi^{*}[d]\left(\Sigma_{Y, t}\right)\right|_{R_{a}},\left.\phi^{*}[d]\left(\mathrm{T}_{Y, t}\right)\right|_{R_{a}}$ with $\left.\Sigma_{X, s}\right|_{R_{a}},\left.\mathrm{~T}_{X, s}\right|_{R_{a}}$ from above, $\Delta_{\phi}$ identifies $\phi^{*}[d]\left(\Sigma_{Y, t}\right), \phi^{*}[d]\left(\mathrm{T}_{Y, t}\right)$ with $\Sigma_{X, s}, \mathrm{~T}_{X, s}$. By our usual argument involving taking disjoint union of two open covers, we see that $\Delta_{\phi}$ is independent of the choice of data $A$ and $\left(R_{a}, U_{a}, f_{a}, i_{a}\right),\left(S_{a}, V_{a}, g_{a}, j_{a}\right), \Phi_{a}$ for $a \in$ $A$. Let $(R, U, f, i),(S, V, g, j), \Phi$ be as in (a). By defining $\Delta_{\phi}$ using data $A,\left(R_{a}, U_{a}, f_{a}, i_{a}\right),\left(S_{a}, V_{a}, g_{a}, j_{a}\right), \Phi_{a}$ with $(R, U, f, i),(S, V, g, j), \Phi$ equal to $\left(R_{a}\right.$, $\left.U_{a}, f_{a}, i_{a}\right),\left(S_{a}, V_{a}, g_{a}, j_{a}\right), \Phi_{a}$ for some $a \in A$, we see that part (a) holds.

For (b), let $x \in X$ with $y=\phi(x) \in Y$ and $z=\psi(z) \in Z$. The proof of Proposition 3.2 in 15 shows we may choose critical charts $(R, U, f, i),(S, V, g, j)$, $(T, W, h, k)$ on $(X, s),(Y, t),(Z, u)$ with $x \in R, y \in \phi(R) \subseteq S, z \in \psi(S) \subseteq T$ of minimal dimensions $\operatorname{dim} U=\operatorname{dim} T_{x} X, \operatorname{dim} V=\operatorname{dim} T_{y} Y, \operatorname{dim} W=\operatorname{dim} T_{z} Z$, and $\Phi: U \rightarrow V, \Psi: V \rightarrow W$ smooth of relative dimensions $d$, $e$ with $f=g \circ \Phi$, $g=h \circ \Psi$ and $\Phi \circ i=j \circ \phi, \Psi \circ j=k \circ \psi$. Consider the diagram of isomorphisms:

$$
\begin{aligned}
& \left.\left.(\psi \circ \phi)\right|_{R} ^{*}[d+e] \longrightarrow(\psi \circ \phi)\right|_{R} ^{*}[d+e]
\end{aligned}
$$

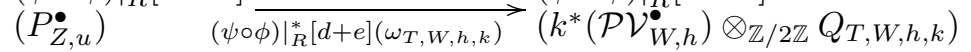

$$
\begin{aligned}
& \left.\Delta_{\psi \circ \phi}\right|_{R}\left(\begin{array}{lr}
\left.\downarrow_{\phi}\right|_{R} ^{*}\left(\Delta_{\psi}\right) \\
\left.\phi\right|_{R} ^{*}[d]\left(P_{Y, t}^{\bullet}\right) \\
\prod_{\left.\Delta_{\phi}\right|_{R}}^{\bullet}
\end{array}\right.
\end{aligned}
$$

The two inner and the outer rectangles commute by (4.6). Also $\alpha_{\Psi \circ \Phi}=$ $\left.\alpha_{\Phi} \circ \phi\right|_{R} ^{*}\left(\alpha_{\Psi}\right)$ is immediate and $\Xi_{\Psi \circ \Phi}=\left.\Xi_{\Phi} \circ \Phi\right|_{\text {Crit }(f)} ^{*}[d]\left(\Xi_{\Psi}\right)$ follows from the definition of $\Xi_{\Phi}$ in Proposition $4.3(\mathrm{~b})$, so the right hand semicircle commutes. Therefore the left hand semicircle commutes. This proves the restriction of (4.7) to $R \subseteq X$. As we can cover $X$ by such open $R$, equation (4.7) follows. 
For part (c), all the facts we have used about perverse sheaves on $\mathbb{C}$-schemes above also hold in the other settings of $l$-adic perverse sheaves on $\mathbb{K}$-schemes, $\mathscr{D}$-modules, and mixed Hodge modules. This completes the proof.

\subsection{Perverse sheaves on Artin stacks}

We first note that because of Proposition 4.1 and Theorem 4.2, any of the theories of perverse sheaves on $\mathbb{C}$-schemes or $\mathbb{K}$-schemes mentioned in $\$ 4.1$ can be extended to Artin $\mathbb{C}$-stacks or Artin $\mathbb{K}$-stacks $X$ in a naïve way, using the philosophy discussed in $\$ 3.2$ and [15, §2.7] of defining sheaves on $X$ in terms of sheaves on schemes $T$ for smooth $t: T \rightarrow X$, in particular Proposition 3.10;

Definition 4.6. Fix one of the theories of perverse sheaves on $\mathbb{K}$-schemes discussed in 84.1 , over an allowed base ring $A$, where we include the special case $\mathbb{K}=\mathbb{C}$ and $A$ is general as in Dimca 7 . Let $X$ be an Artin $\mathbb{K}$-stack, always assumed locally of finite type. We will explain how to define an abelian category $\operatorname{Perv}_{\text {nai }}(X)$ of naïve perverse sheaves on $X$ :

(A) Define an object $\mathcal{P}$ of $\operatorname{Perv}_{\text {nai }}(X)$ to assign

(a) For each $\mathbb{K}$-scheme $T$ and smooth 1-morphism $t: T \rightarrow X$, a perverse sheaf $\mathcal{P}(T, t) \in \operatorname{Perv}(T)$ on $T$ in our chosen $\mathbb{K}$-scheme perverse sheaf theory.

(b) For each 2-commutative diagram in Art $_{\mathbb{K}}$ :

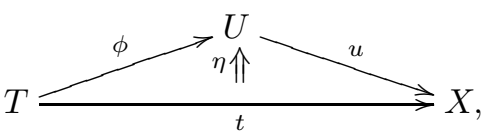

where $T, U$ are $\mathbb{K}$-schemes and $\phi, t, u$ are smooth with $\phi$ of dimension $d$, an isomorphism $\mathcal{P}(\phi, \eta): \phi^{*}[d](\mathcal{P}(U, u)) \rightarrow \mathcal{P}(T, t)$ in $\operatorname{Perv}(T)$.

This data must satisfy the following condition:

(i) For each 2-commutative diagram in $\mathrm{Art}_{\mathbb{K}}$ :

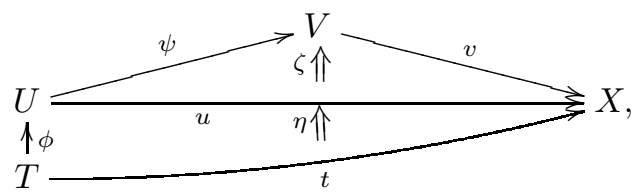

with $T, U, V \mathbb{K}$-schemes and $\phi, \psi, t, u, v$ smooth with $\phi, \psi$ of dimensions $d, e$, we must have

$$
\begin{aligned}
\mathcal{P}\left(\psi \circ \phi,\left(\zeta * \operatorname{id}_{\phi}\right) \odot \eta\right) & =\mathcal{P}(\phi, \eta) \circ \phi^{*}[d](\mathcal{P}(\psi, \zeta)) \quad \text { as morphisms } \\
(\psi \circ \phi)^{*}[d+e](\mathcal{P}(V, v)) & =\phi *[d] \circ \psi^{*}[e](\mathcal{P}(V, v)) \longrightarrow \mathcal{P}(T, t) .
\end{aligned}
$$


(B) Morphisms $\alpha: \mathcal{P} \rightarrow \mathcal{Q}$ of $\operatorname{Perv}_{\text {naï }}(X)$ comprise a morphism $\alpha(T, t)$ : $\mathcal{P}(T, t) \rightarrow \mathcal{Q}(T, t)$ in $\operatorname{Perv}(T)$ for all smooth 1-morphisms $t: T \rightarrow X$ from a scheme $T$, such that for each diagram (4.14) in (b) the following commutes:

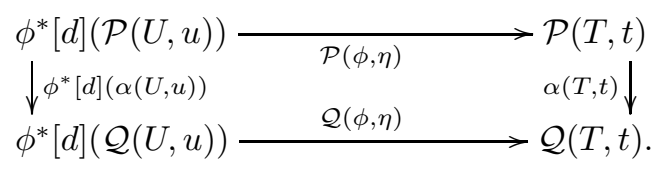

(C) Composition of morphisms $\mathcal{P} \stackrel{\alpha}{\longrightarrow} \mathcal{Q} \stackrel{\beta}{\longrightarrow} \mathcal{R}$ in $\operatorname{Perv}_{\text {naï }}(X)$ is $(\beta \circ \alpha)(T, t)=$ $\beta(T, t) \circ \alpha(T, t)$. Identity morphisms $\operatorname{id}_{\mathcal{P}}: \mathcal{P} \rightarrow \mathcal{P} \operatorname{are~}_{\mathcal{P}}(T, t)=\operatorname{id}_{\mathcal{P}(T, t)}$.

We can also define a category of naïve $\mathscr{D}$-modules on $X$ in the same way.

Remark 4.7. Definition 4.6 for $\mathcal{P}$ is modelled on Proposition 3.10 for $\mathcal{A}$, with the following differences:

(i) $\mathcal{P}(\phi, \eta)$ is an isomorphism always, but $\mathcal{A}(\phi, \eta)$ need only be an isomorphism if $\phi$ is étale. Now $\mathcal{A}$ in Proposition 3.10(A) is called a Cartesian sheaf on $X$ if $\mathcal{A}(\phi, \eta)$ is an isomorphism always. So $\mathcal{P}$ is the perverse analogue of a Cartesian sheaf $\mathcal{A}$ on $X$.

(ii) $\mathcal{P}(\phi, \eta)$ is defined only when $\phi$ is smooth, but $\mathcal{A}(\phi, \eta)$ is defined without requiring $\phi$ smooth. For Cartesian sheaves $\mathcal{A}$ on $X$, it is enough to give the data $\mathcal{A}(T, t), \mathcal{A}(\phi, \eta)$ and check the conditions for $\phi$ smooth; the remaining $\mathcal{A}(\phi, \eta)$ for non-smooth $\phi$ are then determined uniquely.

(iii) Definition [4.6 uses shifted pullbacks $\phi^{*}[d]$ where Proposition 3.10 uses sheaf pullbacks $\phi^{-1}$. This is because of Proposition 4.1.

Using Proposition 4.1, Theorem 4.2 and formal arguments, we can deduce:

(a) For any Artin stack $X, \operatorname{Perv}_{\text {naï }}(X)$ is an abelian category, and if $X$ is a scheme, the functor $\operatorname{Perv}_{\text {naï }}(X) \rightarrow \operatorname{Perv}(X)$ mapping $\mathcal{P} \mapsto \mathcal{P}\left(X, \operatorname{id}_{X}\right)$ is an equivalence of categories with the category $\operatorname{Perv}(X)$ discussed in 4.1

(b) If $\Phi: X \rightarrow Y$ is a 1-morphism of Artin stacks smooth of relative dimension $d$ then as in Proposition 4.1 there is a natural functor $\Phi_{\text {nai }}^{*}[d]$ : $\operatorname{Perv}_{\text {naï }}(Y) \rightarrow \operatorname{Perv}_{\text {naï }}(X)$.

(c) The analogue of Theorem4.2 holds for the categories Perv naï and pullbacks $\Phi_{\text {naii }}^{*}[d]$, taking the $U_{i}, U_{i j}, U_{i j k}$ to be either schemes or stacks.

This 'naïve' model of perverse sheaves on Artin stacks follows from the scheme case in an essentially trivial way, and is sufficient to prove the first part of the main result of this section, Theorem 4.8 below.

However, for a satisfactory theory of perverse sheaves on Artin stacks, we want more: we would like the category $\operatorname{Perv}(X)$ of perverse sheaves on $X$ to be the heart of a t-structure on a triangulated category $D_{c}^{b}(X)$ of 'constructible complexes', which may not be equivalent to $D^{b} \operatorname{Perv}(X)$, and we would like Grothendieck's "six operations on sheaves" $f^{*}, f^{!}, R f_{*}, R f_{!}, \mathcal{R H}$ Hom,$\stackrel{L}{\otimes}$, and Verdier duality operators $\mathbb{D}_{X}$, to act on these ambient categories $D_{c}^{b}(X)$. Other 
than pullbacks $f^{*}, f^{!}$by smooth 1-morphisms $f: X \rightarrow Y$ and operators $\mathbb{D}_{X}$, none of this is obvious using the definition of perverse sheaves Perv naï $(X)$ above.

Thus, the main issue in developing a good theories of perverse sheaves on Artin stacks $X$ is not defining the categories $\operatorname{Perv}(X)$ or $\operatorname{Perv}_{\text {naï }}(X)$ themselves, but defining the categories $D_{c}^{b}(X)$ and the six operations $f^{*}, \ldots, \stackrel{L}{\otimes}$ upon them, and then defining a perverse t-structure on $D_{c}^{b}(X)$ with heart $\operatorname{Perv}(X)$. If (a)(c) above hold for these $D_{c}^{b}(X), \operatorname{Perv}(X)$, it will then be automatic [24, §7] that $\operatorname{Perv}(X) \simeq \operatorname{Perv}_{\text {nai }}(X)$ for $\operatorname{Perv}_{\text {naï }}(X)$ as in Definition 4.6 .

Here are the foundational papers on perverse sheaves and $\mathscr{D}$-modules on Artin stacks known to the authors:

- Laszlo and Olsson 22 24 generalize the Beilinson-Bernstein-Deligne theory of perverse sheaves on $\mathbb{K}$-schemes with finite and $l$-adic coefficients [1] to Artin stacks. In [24, §7] they show that $\operatorname{Perv}(X)$ is equivalent to the category $\operatorname{Perv}_{\text {nai }}(X)$ in Definition 4.6.

- Liu and Zheng [25,26] develop a theory of perverse sheaves on higher Artin stacks using Lurie's $\infty$-categories, and show it is equivalent to Laszlo and Olsson's version for ordinary Artin stacks.

- Gaitsgory and Rozenblyum [10] construct a theory of crystals on (derived) schemes and stacks $\boldsymbol{X}$. For classical schemes $X$, the categories of crystals and $\mathscr{D}$-modules on $X$ are equivalent, so the authors argue that $\mathscr{D}$-modules on (derived) stacks should be defined to be crystals. The six functor formalism for crystals was not complete at the time of writing.

- In a brief note, for an Artin $\mathbb{C}$-stack $X$, Paulin 31 proposes definitions of constructible complexes $D_{c}^{b}(X)$ over $A=\mathbb{C}$, with its perverse t-structure, and (for smooth $X$ ) of the derived category $D_{r h}^{b}(X)$ of $\mathscr{D}$-modules on $X$ with t-structure, claims the six functor formalism holds, and proves a 'Riemann-Hilbert' equivalence of these categories with t-structures.

\subsection{The main result}

Here is the main result of this section, the analogue of Theorem 4.4 from 3 . Apart from the material in our previous papers [3, 15] and general properties of perverse sheaves on Artin stacks, the only extra ingredient is Proposition 4.5 .

We state Theorem 4.8 and Corollaries 4.9, 4.10 using Laszlo and Olsson's $l$-adic perverse sheaves on Artin stacks [22 24], but they would also work for any other theory of perverse sheaves, or $\mathscr{D}$-modules, or mixed Hodge modules, on Artin stacks, which has the expected package of properties discussed in 4.3 .

Theorem 4.8. Let $(X, s)$ be an oriented d-critical stack over $\mathbb{K}$ (allowing $\mathbb{K}=$ $\mathbb{C})$ with orientation $K_{X, s}^{1 / 2}$. Fix a theory of perverse sheaves on $\mathbb{K}$-schemes from 4.1. and let $\operatorname{Perv}_{\text {naï }}(X)$ be the corresponding category of naïve perverse sheaves on $X$ from Definition 4.6. Then we may define $\mathcal{P}_{X, s} \in \operatorname{Perv}_{\text {naï }}(X)$ and Verdier duality and monodromy isomorphisms

$$
\Sigma_{X, s}: \mathcal{P}_{X, s} \longrightarrow \mathbb{D}_{X}\left(\mathcal{P}_{X, s}\right), \quad \mathrm{T}_{X, s}: \mathcal{P}_{X, s} \longrightarrow \mathcal{P}_{X, s},
$$

as follows: 
(a) If $t: T \rightarrow X$ is smooth with $T$ a $\mathbb{K}$-scheme, so that $(T, s(T, t))$ is an algebraic d-critical locus with natural orientation $K_{T, s(T, t)}^{1 / 2}$ as in Lemma 3.17 then $\mathcal{P}_{X, s}(T, t)=P_{T, s(T, t)}^{\bullet}$ in $\operatorname{Perv}(T)$, where $P_{T, s(T, t)}^{\bullet}$ is the perverse sheaf on the oriented algebraic d-critical locus $(T, s(T, t))$ over $\mathbb{K}$ given by Theorem 4.4. Also $\Sigma_{X, s}(T, t)=\Sigma_{T, s(T, t)}$ and $\mathrm{T}_{X, s}(T, t)=\mathrm{T}_{T, s(T, t)}$.

(b) For each 2-commutative diagram in $\operatorname{Art}_{\mathbb{K}}$

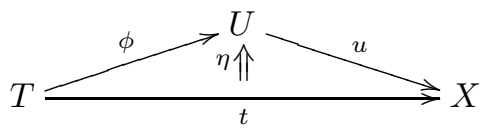

with $T, U \mathbb{K}$-schemes and $\phi, t, u$ smooth with $\phi$ of dimension $d$, we have

$$
\begin{gathered}
\mathcal{P}_{X, s}(\phi, \eta)=\Delta_{\phi}: \phi^{*}[d]\left(\mathcal{P}_{X, s}(U, u)\right)=\phi^{*}[d]\left(P_{U, s(U, u)}^{\bullet}\right) \\
\longrightarrow \mathcal{P}_{X, s}(T, t)=P_{T, s(T, t)}^{\bullet},
\end{gathered}
$$

where $\Delta_{\phi}$ is as in Proposition 4.5 .

If we work with perverse sheaves on $\mathbb{K}$-schemes in the sense of 1 over a base ring $A$ with either char $A>0$ coprime to char $\mathbb{K}$, or $A=\mathbb{Z}_{l}, \mathbb{Q}_{l}$ or $\overline{\mathbb{Q}}_{l}$ with l coprime to char $\mathbb{K}$, then $\operatorname{Perv}_{\text {naï }}(X) \simeq \operatorname{Perv}(X)$ as in 4.3 , where $\operatorname{Perv}(X) \subset D_{c}^{b}(X)$ is the category of perverse sheaves on $X$ over $A$ defined by Laszlo and Olsson 22 24. Thus $\mathcal{P}_{X, s}$ corresponds to $\check{P}_{X, s}^{\bullet} \in \operatorname{Perv}(X)$ unique up to canonical isomorphism, and $\Sigma_{X, s}, \mathrm{~T}_{X, s}$ correspond to isomorphisms

$$
\check{\Sigma}_{X, s}: \check{P}_{X, s}^{\bullet} \longrightarrow \mathbb{D}_{X}\left(\check{P}_{X, s}^{\bullet}\right), \quad \check{\mathrm{T}}_{X, s}: \check{P}_{X, s}^{\bullet} \longrightarrow \check{P}_{X, s}^{\bullet} \quad \text { in } \operatorname{Perv}(X) \text {. }
$$

The analogue of the above will also hold in any other theory of perverse sheaves or $\mathscr{D}$-modules on schemes and Artin stacks with the package of properties discussed in $\$ 4.3$, including the six operations $f^{*}, f^{!}, R f_{*}, R f_{!}, \mathcal{R H o m}, \stackrel{L}{\otimes}$, Verdier duality $\mathbb{D}_{X}$, and descent in the smooth topology as in Theorem 4.2 .

Proof. Proposition 4.5(b) implies that the data $\mathcal{P}_{X, s}(T, t), \mathcal{P}_{X, s}(\phi, \eta)$ in (a),(b) satisfy Definition 4.6(A)(i). Thus $\mathcal{P}_{X, s}$ is an object of $\operatorname{Perv}_{\text {nai }}(X)$. Similarly, the last part of Proposition 4.5(a) implies that $\Sigma_{X, s}, \mathrm{~T}_{X, s}$ are morphisms in $\operatorname{Perv}_{\text {nai }}(X)$. The last part is immediate from the discussion of 4.3 .

Combining Theorems 2.10, 3.18 and 4.8 and Corollary 3.19 yields:

Corollary 4.9. Let $\mathbb{K}$ be an algebraically closed field of characteristic zero, $(\boldsymbol{X}, \omega)$ a-1-shifted symplectic derived Artin $\mathbb{K}$-stack, and $X=t_{0}(\boldsymbol{X})$ the associated classical Artin $\mathbb{K}$-stack. Suppose we are given a square root $\left.\operatorname{det}\left(\mathbb{L}_{\boldsymbol{X}}\right)\right|_{X} ^{1 / 2}$.

Then working in l-adic perverse sheaves on stacks 22 24, we may define a perverse sheaf $\tilde{P}_{\boldsymbol{X}, \omega}^{\bullet}$ on $X$ uniquely up to canonical isomorphism, and Verdier duality and monodromy isomorphisms $\check{\Sigma}_{\boldsymbol{X}, \omega}: \check{P}_{\boldsymbol{X}, \omega}^{\bullet} \rightarrow \mathbb{D}_{X}\left(\check{P}_{\boldsymbol{X}, \omega}^{\bullet}\right)$ and $\check{\mathrm{T}}_{\boldsymbol{X}, \omega}$ : $\check{P}_{\boldsymbol{X}, \omega} \rightarrow \check{P}_{\boldsymbol{X}, \omega}$. These are characterized by the fact that given a diagram

$$
\boldsymbol{U}=\operatorname{Crit}\left(f: U \rightarrow \mathbb{A}^{1}\right) \longleftarrow i \quad \boldsymbol{X} \underset{\boldsymbol{C}}{\longrightarrow}
$$


such that $U$ is a smooth $\mathbb{K}$-scheme, $\boldsymbol{\varphi}$ smooth of dimension $n, \mathbb{L}_{\boldsymbol{V} / \boldsymbol{U}} \simeq \mathbb{T}_{\boldsymbol{V} / \boldsymbol{X}}[2]$, $\boldsymbol{\varphi}^{*}\left(\omega_{\boldsymbol{X}}\right) \sim \boldsymbol{i}^{*}\left(\omega_{\boldsymbol{U}}\right)$ for $\omega_{\boldsymbol{U}}$ the natural -1 -shifted symplectic structure on $\boldsymbol{U}=$ $\operatorname{Crit}\left(f: U \rightarrow \mathbb{A}^{1}\right)$, and $\varphi^{*}\left(\left.\operatorname{det}\left(\mathbb{L}_{\boldsymbol{X}}\right)\right|_{X} ^{1 / 2}\right) \cong i^{*}\left(K_{U}\right) \otimes \Lambda^{n} \mathbb{T}_{\boldsymbol{V} / \boldsymbol{X}}$, then $\varphi^{*}\left(\check{P}_{\boldsymbol{X}, \omega}^{\bullet}\right)[n]$, $\varphi^{*}\left(\check{\Sigma}_{\boldsymbol{X}, \omega}^{\bullet}\right)[n], \varphi^{*}\left(\check{\mathrm{T}}_{\boldsymbol{X}, \omega}^{\bullet}\right)[n]$ are canonically isomorphic to $i^{*}\left(\mathcal{P} \mathcal{V}_{U, f}\right), i^{*}\left(\sigma_{U, f}\right)$, $i^{*}\left(\tau_{U, f}\right)$, for $\mathcal{P} \mathcal{V}_{U, f}, \sigma_{U, f}, \tau_{U, f}$ as in $\$ 4.1$.

Corollary 4.10. Let $Y$ be a Calabi-Yau 3-fold over an algebraically closed field $\mathbb{K}$ of characteristic zero, and $\mathcal{M}$ a classical moduli $\mathbb{K}$-stack of coherent sheaves $F$ in $\operatorname{coh}(Y)$, or of complexes $F^{\bullet}$ in $D^{b} \operatorname{coh}(Y)$ with $\operatorname{Ext}^{<0}\left(F^{\bullet}, F^{\bullet}\right)=0$, with obstruction theory $\phi: \mathcal{E}^{\bullet} \rightarrow \mathbb{L}_{\mathcal{M}}$. Suppose we are given a square root $\operatorname{det}\left(\mathcal{E}^{\bullet}\right)^{1 / 2}$.

Then working in l-adic perverse sheaves on stacks [22 24, we may define a natural perverse sheaf $\check{P}_{\mathcal{M}}^{\mathbf{M}} \in \operatorname{Perv}(\mathcal{M})$, and Verdier duality and monodromy isomorphisms $\check{\Sigma}_{\mathcal{M}}: \check{P}_{\mathcal{M}} \rightarrow \mathbb{D}_{\mathcal{M}}\left(\check{P}_{\mathcal{M}}^{\bullet}\right)$ and $\check{\mathrm{T}}_{\mathcal{M}}: \check{P}_{\mathcal{M}} \rightarrow \check{P}_{\mathcal{M}}^{\bullet}$. The pointwise Euler characteristic of $\check{P}_{\mathcal{M}}$ is the Behrend function $\nu_{\mathcal{M}}$ of $\mathcal{M}$ from Joyce and Song [16, §4], so that $\check{P}_{\mathcal{M}}^{\bullet}$ is in effect a categorification of the Donaldson-Thomas theory of $\mathcal{M}$.

Example 4.11. Suppose an algebraic $\mathbb{K}$-group $G$ acts on a $\mathbb{K}$-scheme $T$ with action $\mu: G \times T \rightarrow T$, and write $X$ for the quotient Artin $\mathbb{K}$-stack $[T / G]$, and $t: T \rightarrow[T / G]$ for the natural quotient 1-morphism.

As in Example 3.14 there is a 1-1 correspondence between d-critical structures $s$ on $X=[T / G]$ and $G$-invariant d-critical structures $s^{\prime}$ on $T$, such that $s^{\prime}=s(T, t)$. Also, from Lemma 3.17 we see that there is a 1-1 correspondence between orientations $K_{X, s}^{1 / 2}$ for $(X, s)$, and $G$-invariant orientations $K_{T, s^{\prime}}^{1 / 2}$ for $\left(T, s^{\prime}\right)$, given by $K_{T, s^{\prime}}^{1 / 2}=\left.K_{X, s}^{1 / 2}\left(T^{\text {red }}, t^{\text {red }}\right) \otimes\left(\Lambda^{\text {top }} \mathbb{L}_{T / X}\right)\right|_{T^{\text {red }}}$.

Choose such $s, s^{\prime}, K_{X, s}^{1 / 2}, K_{T, s^{\prime}}^{1 / 2}$, so that Theorems 4.4 and 4.8 give perverse sheaves $P_{T, s^{\prime}}^{\bullet}, \check{P}_{X, s}^{\bullet}$ on $T, X$. We would like to relate the hypercohomologies $\mathbb{H}^{*}\left(T, P_{T, s^{\prime}}^{\bullet}\right), \mathbb{H}^{*}\left(X, \check{P}_{X, s}^{\bullet}\right)$. We have $t^{*}\left(\check{P}_{X, s}^{\bullet}\right)[\operatorname{dim} G] \cong P_{T, s^{\prime}}^{\bullet}$ and thus

$$
R^{q} t_{*} P_{T, s^{\prime}}^{\bullet} \cong R^{q} t_{*} t^{*}\left(\check{P}_{X, s}^{\bullet}\right)[\operatorname{dim} G] \cong \check{P}_{X, s}^{\bullet} \otimes_{A_{X}} R^{q} t_{*}\left(A_{T}\right)[\operatorname{dim} G],
$$

where $A_{T}$ is the constant sheaf on $T$ with fibre the base ring $A$. Therefore, the Leray-Serre spectral sequence for the fibration $t: T \rightarrow X$ with fibre $G$, twisted by $\check{P}_{X, s}^{\bullet}$, can be interpreted as a spectral sequence

$$
E^{\bullet, \bullet} \Longrightarrow \mathbb{H}^{\bullet}\left(T, P_{T, s^{\prime}}^{\bullet}\right) \quad \text { with } \quad E_{2}^{p, q}=\mathbb{H}^{p}\left(X, \check{P}_{X, s}^{\bullet} \otimes_{A_{X}} R^{q} t_{*}\left(A_{T}\right)[\operatorname{dim} G]\right),
$$

where $R^{q} t_{*}\left(A_{T}\right)[\operatorname{dim} G]$ is locally constant on $X$ with fibre $H^{q-\operatorname{dim} G}(G, A)$.

We also have a projection $\pi: X=[T / G] \rightarrow[* / G]$ for $*=$ Spec $\mathbb{K}$ with fibre $T$. The Leray-Serre spectral sequence for $\pi$ gives a spectral sequence

$$
E^{\bullet, \bullet} \Longrightarrow \mathbb{H}^{\bullet}\left(X, \check{P}_{X, s}^{\bullet}\right) \quad \text { with } \quad E_{2}^{p, q}=\mathbb{H}^{p}\left([* / G], \mathbb{H}^{q+\operatorname{dim} G}\left(T, P_{T, s^{\prime}}^{\bullet}\right)\right)
$$

If $G$ is finite we can consider the $\mathbb{H}^{*}\left(T, P_{T, s^{\prime}}^{\bullet}\right)$ as $G$-modules and $\mathbb{H}^{*}([* / G],-)$ as group cohomology $H_{\mathrm{grp}}^{*}(G,-)$, giving a spectral sequence

$$
H_{\mathrm{grp}}^{p}\left(G, \mathbb{H}^{q}\left(T, P_{T, s^{\prime}}^{\bullet}\right)\right) \Longrightarrow \mathbb{H}^{p+q}\left(X, \check{P}_{X, s}^{\bullet}\right) .
$$


Example 4.12. Suppose that $\left(\boldsymbol{X}, \omega_{\boldsymbol{X}}\right)$ is an oriented -1-shifted symplectic derived Artin $\mathbb{K}$-stack, and a finite group $G$ acts on $\boldsymbol{X}$ preserving $\omega_{\boldsymbol{X}}$ and the orientation. Let $\boldsymbol{Y}$ be the derived Artin $\mathbb{K}$-stack $[\boldsymbol{X} / G]$ equipped with the natural quotient -1 -shifted symplectic structure $\omega_{\boldsymbol{Y}}$ and orientation, and write $\boldsymbol{f}: \boldsymbol{X} \rightarrow \boldsymbol{Y}$ for the étale quotient morphism of derived Artin $\mathbb{K}$-stacks. Then we have $\boldsymbol{f}^{*}\left(\omega_{\boldsymbol{Y}}\right) \sim \omega_{\boldsymbol{X}}$ and $f^{*}\left(\check{P}_{\boldsymbol{Y}, \omega_{\boldsymbol{Y}}}^{\bullet}\right) \cong \check{P}_{\boldsymbol{X}, \omega_{\boldsymbol{X}}}^{\bullet}$, and therefore

$$
R^{q} f_{*} P_{\boldsymbol{X}, \omega_{\boldsymbol{X}}}^{\bullet} \cong R^{q} f_{*} f^{*}\left(\check{P}_{\boldsymbol{Y}, \omega_{\boldsymbol{Y}}}^{\bullet}\right) \cong \check{P}_{\boldsymbol{Y}, \omega_{\boldsymbol{Y}}}^{\bullet} \otimes_{A_{\boldsymbol{Y}}} R^{q} f_{*}\left(A_{\boldsymbol{X}}\right) .
$$

Therefore, the Leray-Serre spectral sequence for the fibration $\boldsymbol{f}: \boldsymbol{X} \rightarrow \boldsymbol{Y}$ with fibre $G$ can be interpreted as a spectral sequence

$$
E^{\bullet, \bullet} \Longrightarrow \mathbb{H}^{\bullet}\left(X, \check{P}_{\boldsymbol{X}, \omega_{\boldsymbol{X}}}^{\bullet}\right) \quad \text { with } \quad E_{2}^{p, q}=\mathbb{H}^{p}\left(Y, \check{P}_{\boldsymbol{Y}, \omega_{\boldsymbol{Y}}}^{\bullet} \otimes_{A_{\boldsymbol{Y}}} R^{q} f_{*}\left(A_{\boldsymbol{X}}\right)\right)
$$

Since $G$ is finite, only $q=0$ contributes and we get isomorphisms

$$
\mathbb{H}^{p}\left(X, \check{P}_{\boldsymbol{X}, \omega_{\boldsymbol{X}}}^{\bullet}\right) \cong \mathbb{H}^{p}\left(Y, \check{P}_{\boldsymbol{Y}, \omega_{\boldsymbol{Y}}}^{\bullet} \otimes_{A_{\boldsymbol{Y}}} f_{*}\left(A_{\boldsymbol{X}}\right)\right) .
$$

We also have a projection $\boldsymbol{\pi}: \boldsymbol{Y}=[\boldsymbol{X} / G] \rightarrow[* / G]$ for $*=$ Spec $\mathbb{K}$ with fibre $\boldsymbol{X}$. The Leray-Serre spectral sequence for $\pi$ gives a spectral sequence

$$
E^{\bullet, \bullet} \Longrightarrow \mathbb{H}^{\bullet}\left(Y, \check{P}_{\boldsymbol{Y}, \omega_{\boldsymbol{Y}}}\right) \quad \text { with } \quad E_{2}^{p, q}=\mathbb{H}^{p}\left([* / G], \mathbb{H}^{q}\left(X, \check{P}_{\boldsymbol{X}, \omega_{\boldsymbol{X}}}^{\bullet}\right)\right)
$$

We consider the $\mathbb{H}^{*}\left(X, \check{P}_{\boldsymbol{X}, \omega_{\boldsymbol{X}}}^{\bullet}\right)$ as $G$-modules and observe $\mathbb{H}^{*}([* / G],-)$ is the same as group cohomology $H_{\mathrm{grp}}^{*}(G,-)$, giving a spectral sequence

$$
H_{\mathrm{grp}}^{p}\left(G, \mathbb{H}^{q}\left(X, \check{P}_{\boldsymbol{X}, \omega_{\boldsymbol{X}}}^{\bullet}\right)\right) \Longrightarrow \mathbb{H}^{\bullet}\left(Y, \check{P}_{\boldsymbol{Y}, \omega_{\boldsymbol{Y}}}\right) .
$$

\section{Motives on d-critical stacks}

We now extend the results of [5] to d-critical stacks. Our main result Theorem 5.14 in $\$ 5.4$, proved in $\$ 5.5$, states that an oriented d-critical stack $(X, s)$ which is of finite type and locally a global quotient carries a natural motive in a certain ring of motives $\overline{\mathcal{M}}_{X}^{\text {st, } \hat{\mu}}$, defined in $\$ 5.3$

In this section, $\mathbb{K}$ is an algebraically closed field of characteristic zero, and all $\mathbb{K}$-schemes and Artin $\mathbb{K}$-stacks will be assumed to be of finite type unless we explicitly say otherwise. From after Proposition [5.10, all Artin $\mathbb{K}$-stacks will also be assumed to have affine geometric stabilizers.

\subsection{Rings of motives on $\mathbb{K}$-schemes}

We begin by defining rings of motives $K_{0}\left(\operatorname{Sch}_{X}\right), \mathcal{M}_{X}, K_{0}^{\hat{\mu}}\left(\operatorname{Sch}_{X}\right), \mathcal{M}_{X}^{\hat{\mu}}$ for a $\mathbb{K}$-scheme $X$. Some references are Denef and Loeser [6], Looijenga [27, and Joyce [14]. Our notation follows Bussi, Joyce and Meinhardt [5].

Definition 5.1. Let $X$ be a $\mathbb{K}$-scheme (always assumed of finite type). Consider pairs $(R, \rho)$, where $R$ is a $\mathbb{K}$-scheme and $\rho: R \rightarrow X$ is a morphism. Call two pairs $(R, \rho),\left(R^{\prime}, \rho^{\prime}\right)$ equivalent if there is an isomorphism $\iota: R \rightarrow R^{\prime}$ with $\rho=\rho^{\prime} \circ \iota$. Write $[R, \rho]$ for the equivalence class of $(R, \rho)$. If $(R, \rho)$ is a pair 
and $S$ is a closed $\mathbb{K}$-subscheme of $R$ then $\left(S,\left.\rho\right|_{S}\right),\left(R \backslash S,\left.\rho\right|_{R \backslash S}\right)$ are pairs of the same kind. Define the Grothendieck ring $K_{0}\left(\operatorname{Sch}_{X}\right)$ of the category $\operatorname{Sch}_{X}$ of $\mathbb{K}$-schemes over $X$ to be the abelian group generated by equivalence classes $[R, \rho]$, with the relation that for each closed $\mathbb{K}$-subscheme $S$ of $R$ we have

$$
[R, \rho]=\left[S,\left.\rho\right|_{S}\right]+\left[R \backslash S,\left.\rho\right|_{R \backslash S}\right] .
$$

Define a product '.' on $K_{0}\left(\operatorname{Sch}_{X}\right)$ by

$$
[R, \rho] \cdot[S, \sigma]=\left[R \times_{\rho, X, \sigma} S, \rho \circ \pi_{R}\right] .
$$

This is compatible with (5.1), and extends to a biadditive, commutative, associative product $\cdot: K_{0}\left(\operatorname{Sch}_{X}\right) \times K_{0}\left(\operatorname{Sch}_{X}\right) \rightarrow K_{0}\left(\operatorname{Sch}_{X}\right)$. It makes $K_{0}\left(\operatorname{Sch}_{X}\right)$ into a commutative ring, with identity $1_{X}=\left[X, \mathrm{id}_{X}\right]$.

Define $\mathbb{L}=\left[\mathbb{A}^{1} \times X, \pi_{X}\right]$ in $K_{0}\left(\operatorname{Sch}_{X}\right)$. We denote by

$$
\mathcal{M}_{X}=K_{0}\left(\operatorname{Sch}_{X}\right)\left[\mathbb{L}^{-1}\right]
$$

the ring obtained from $K_{0}\left(\operatorname{Sch}_{X}\right)$ by inverting $\mathbb{L}$. When $X=$ Spec $\mathbb{K}$ we write $K_{0}\left(\mathrm{Sch}_{\mathbb{K}}\right), \mathcal{M}_{\mathbb{K}}$ instead of $K_{0}\left(\operatorname{Sch}_{X}\right), \mathcal{M}_{X}$.

The external tensor products $\otimes: K_{0}\left(\operatorname{Sch}_{X}\right) \times K_{0}\left(\operatorname{Sch}_{Y}\right) \rightarrow K_{0}\left(\operatorname{Sch}_{X \times Y}\right)$ and $\otimes: \mathcal{M}_{X} \times \mathcal{M}_{Y} \rightarrow \mathcal{M}_{X \times Y}$ are

$$
\left(\sum_{i \in I} c_{i}\left[R_{i}, \rho_{i}\right]\right) \otimes\left(\sum_{j \in J} d_{j}\left[S_{j}, \sigma_{j}\right]\right)=\sum_{i \in I, j \in J} c_{i} d_{j}\left[R_{i} \times S_{j}, \rho_{i} \times \sigma_{j}\right],
$$

for finite $I, J$. They are biadditive, commutative, and associative. Taking $Y=$ Spec $\mathbb{K}$, we see that $\otimes$ makes $K_{0}\left(\operatorname{Sch}_{X}\right), \mathcal{M}_{X}$ into modules over $K_{0}\left(\operatorname{Sch}_{\mathbb{K}}\right), \mathcal{M}_{\mathbb{K}}$.

Let $\phi: X \rightarrow Y$ be a morphism of $\mathbb{K}$-schemes. Define the pushforwards $\phi_{*}: K_{0}\left(\operatorname{Sch}_{X}\right) \rightarrow K_{0}\left(\operatorname{Sch}_{Y}\right)$ and $\phi_{*}: \mathcal{M}_{X} \rightarrow \mathcal{M}_{Y}$ by

$$
\phi_{*}: \sum_{i=1}^{n} c_{i}\left[R_{i}, \rho_{i}\right] \longmapsto \sum_{i=1}^{n} c_{i}\left[R_{i}, \phi \circ \rho_{i}\right] .
$$

Define pullbacks $\phi^{*}: K_{0}\left(\operatorname{Sch}_{Y}\right) \rightarrow K_{0}\left(\operatorname{Sch}_{X}\right)$ and $\phi^{*}: \mathcal{M}_{Y} \rightarrow \mathcal{M}_{X}$ by

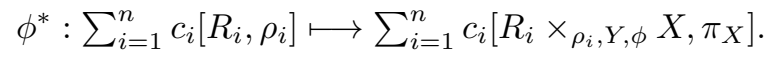

Pushforwards and pullbacks have the obvious functoriality properties. As in [14, Th. 3.5], pushforwards and pullbacks commute in Cartesian squares, that is, if

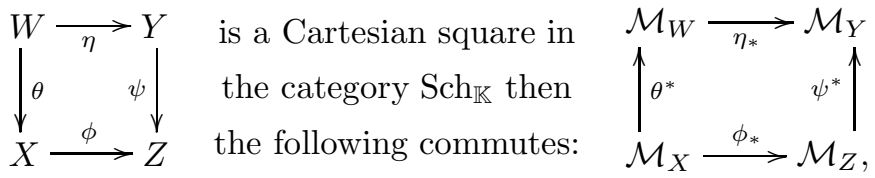

and the analogue holds for $K_{0}\left(\operatorname{Sch}_{W}\right), \ldots, K_{0}\left(\operatorname{Sch}_{Z}\right)$.

Definition 5.2. For $n=1,2, \ldots$, write $\mu_{n}$ for the group of all $n^{\text {th }}$ roots of unity in $\mathbb{K}$, which is assumed algebraically closed of characteristic zero, so that $\mu_{n} \cong \mathbb{Z}_{n}$. The $\mu_{n}$ form a projective system, with respect to the maps $\mu_{n d} \rightarrow \mu_{n}$ mapping $x \mapsto x^{d}$. Define the group $\hat{\mu}$ to be the projective limit of the $\mu_{n}$. 
Let $R$ be a $\mathbb{K}$-scheme. A good $\mu_{n}$-action on $R$ is a group action $r_{n}: \mu_{n} \times R \rightarrow$ $R$ such that such that each orbit is contained in an open affine subscheme of $R$ and $\rho \circ r_{n}(\gamma) \cong \rho$ for all $\gamma \in \mu_{n}$. A good $\hat{\mu}$-action on $R$ is a group action $\hat{r}: \hat{\mu} \times R \rightarrow R$ which factors through a good $\mu_{n}$-action, for some $n$. We will write $\hat{\iota}: \hat{\mu} \times R \rightarrow R$ for the trivial $\hat{\mu}$-action on $R$, which is automatically good.

Consider triples $(R, \rho, \hat{r})$, where $R$ is a $\mathbb{K}$-scheme, $\rho: R \rightarrow X$ a morphism, and $\hat{r}: \hat{\mu} \times R \rightarrow R$ a good $\hat{\mu}$-action on $R$. Call two such triples $(R, \rho, \hat{r}),\left(R^{\prime}, \rho^{\prime}, \hat{r}^{\prime}\right)$ equivalent if there exists a $\hat{\mu}$-equivariant isomorphism $\iota: R \rightarrow R^{\prime}$ with $\rho=\rho^{\prime} \circ \iota$. Write $[R, \rho, \hat{r}]$ for the equivalence class of $(R, \rho, \hat{r})$.

The monodromic Grothendieck group $K_{0}^{\hat{\mu}}\left(\mathrm{Sch}_{X}\right)$ is the abelian group generated by such equivalence classes $[R, \rho, \hat{r}]$, with the relations:

(i) for each closed $\hat{\mu}$-invariant $\mathbb{K}$-subscheme $S$ of $R$, we have

$$
[R, \rho, \hat{r}]=\left[S,\left.\rho\right|_{S},\left.\hat{r}\right|_{S}\right]+\left[R \backslash S,\left.\rho\right|_{R \backslash S},\left.\hat{r}\right|_{R \backslash S}\right] ;
$$

(ii) given $\left[R_{1}, \rho_{1}, \hat{r}_{1}\right],\left[R_{2}, \rho_{2}, \hat{r}_{2}\right]$ with $\pi: R_{2} \rightarrow R_{1}$ a $\hat{\mu}$-equivariant vector bundle of rank $d$ over $R_{1}$ and $\rho_{2}=\rho_{1} \circ \pi$, then

$$
\left[R_{2}, \rho_{2}\right]=\left[R_{1} \times \mathbb{A}^{d}, \rho_{1} \circ \pi, \hat{r}_{1} \times \hat{\imath}\right] .
$$

There is a natural biadditive product '.' on $K_{0}^{\hat{\mu}}\left(\operatorname{Sch}_{X}\right)$ given by

$$
[R, \rho, \hat{r}] \cdot[S, \sigma, \hat{s}]=\left[R \times_{\rho, X, \sigma} S, \rho \circ \pi_{R}, \hat{r} \times \hat{s}\right],
$$

making $K_{0}^{\hat{\mu}}\left(\operatorname{Sch}_{X}\right)$ into a commutative ring, with identity $1_{X}=\left[X, \operatorname{id}_{X}, \hat{\iota}\right]$.

Define $\mathbb{L}=\left[\mathbb{A}^{1} \times X, \pi_{X}, \hat{\iota}\right]$ in $K_{0}^{\hat{\mu}}\left(\operatorname{Sch}_{X}\right)$. We denote by

$$
\mathcal{M}_{X}^{\hat{\mu}}=K_{0}^{\hat{\mu}}\left(\operatorname{Sch}_{X}\right)\left[\mathbb{L}^{-1}\right]
$$

the ring obtained from $K_{0}^{\hat{\mu}}\left(\operatorname{Sch}_{X}\right)$ by inverting $\mathbb{L}$. When $X=$ Spec $\mathbb{K}$ we write $K_{0}^{\hat{\mu}}\left(\mathrm{Sch}_{\mathbb{K}}\right), \mathcal{M}_{\mathbb{K}}^{\hat{\mu}}$ instead of $K_{0}^{\hat{\mu}}\left(\operatorname{Sch}_{X}\right), \mathcal{M}_{X}^{\hat{\mu}}$.

The external tensor products $\otimes: K_{0}^{\hat{\mu}}\left(\operatorname{Sch}_{X}\right) \times K_{0}^{\hat{\mu}}\left(\operatorname{Sch}_{Y}\right) \rightarrow K_{0}^{\hat{\mu}}\left(\operatorname{Sch}_{X \times Y}\right)$ and $\otimes: \mathcal{M}_{X}^{\hat{\mu}} \times \mathcal{M}_{Y}^{\hat{\mu}} \rightarrow \mathcal{M}_{X \times Y}^{\hat{\mu}}$ are

$$
\left(\sum_{i \in I} c_{i}\left[R_{i}, \rho_{i}, \hat{r}_{i}\right]\right) \otimes\left(\sum_{j \in J} d_{j}\left[S_{j}, \sigma_{j}, \hat{s}_{j}\right]\right)=\sum_{i \in I, j \in J} c_{i} d_{j}\left[R_{i} \times S_{j}, \rho_{i} \times \sigma_{j}, \hat{r}_{i} \times \hat{s}_{j}\right],
$$

for finite $I, J$. Pushforwards $\phi_{*}$ and pullbacks $\phi^{*}$ are defined for $K_{0}^{\hat{\mu}}\left(\operatorname{Sch}_{X}\right), \mathcal{M}_{X}^{\hat{\mu}}$ in the obvious way, and the analogue of (5.7) holds.

There are natural morphisms of commutative rings

$$
\begin{aligned}
i_{X}: K_{0}\left(\operatorname{Sch}_{X}\right) & \longrightarrow K_{0}^{\hat{\mu}}\left(\operatorname{Sch}_{X}\right), \quad i_{X}: \mathcal{M}_{X} \longrightarrow \mathcal{M}_{X}^{\hat{\mu}}, \\
\Pi_{X}: K_{0}^{\hat{\mu}}\left(\operatorname{Sch}_{X}\right) & \longrightarrow K_{0}\left(\operatorname{Sch}_{X}\right), \quad \Pi_{X}: \mathcal{M}_{X}^{\hat{\mu}} \longrightarrow \mathcal{M}_{X},
\end{aligned}
$$

given by $i_{X}:[R, \rho] \mapsto[R, \rho, \hat{\imath}]$ and $\Pi_{X}:[R, \rho, \hat{r}] \mapsto[R, \rho]$.

Following Looijenga [27, §7] and Denef and Loeser [6, §5], we introduce a second multiplication ' $\odot$ ' on $K_{0}^{\hat{\mu}}\left(\operatorname{Sch}_{X}\right), \mathcal{M}_{X}^{\hat{\mu}}$ (written ' $*$ ' in [6, 27] ). 
Definition 5.3. Let $X$ be a $\mathbb{K}$-scheme and $[R, \rho, \hat{r}],[S, \sigma, \hat{s}]$ be generators of $K_{0}^{\hat{\mu}}\left(\operatorname{Sch}_{X}\right)$. Then there exists $n \geqslant 1$ such that the $\hat{\mu}$-actions $\hat{r}, \hat{s}$ on $R, S$ factor through $\mu_{n}$-actions $r_{n}, s_{n}$. Define $J_{n}$ to be the Fermat curve

$$
J_{n}=\left\{(t, u) \in\left(\mathbb{A}^{1} \backslash\{0\}\right)^{2}: t^{n}+u^{n}=1\right\} .
$$

Let $\mu_{n} \times \mu_{n}$ act on $J_{n} \times\left(R \times_{X} S\right)$ by

$$
\left(\alpha, \alpha^{\prime}\right) \cdot((t, u),(v, w))=\left(\left(\alpha \cdot t, \alpha^{\prime} \cdot u\right),\left(r_{n}(\alpha)(v), s_{n}\left(\alpha^{\prime}\right)(w)\right)\right) .
$$

Write $J_{n}(R, S)=\left(J_{n} \times\left(R \times \times_{X} S\right)\right) /\left(\mu_{n} \times \mu_{n}\right)$ for the quotient $\mathbb{K}$-scheme, and define a $\mu_{n}$-action $v_{n}$ on $J_{n}(R, S)$ by

$$
v_{n}(\alpha)((t, u), v, w)\left(\mu_{n} \times \mu_{n}\right)=((\alpha \cdot t, \alpha \cdot u), v, w)\left(\mu_{n} \times \mu_{n}\right) .
$$

Let $\hat{v}$ be the induced good $\hat{\mu}$-action on $J_{n}(R, S)$, and set

$$
[R, \rho, \hat{r}] \odot[S, \sigma, \hat{s}]=(\mathbb{L}-1) \cdot\left[\left(R \times_{X} S\right) / \mu_{n}, \hat{\iota}\right]-\left[J_{n}(R, S), \hat{v}\right]
$$

in $K_{0}^{\hat{\mu}}\left(\operatorname{Sch}_{X}\right)$ and $\mathcal{M}_{X}^{\hat{\mu}}$. This turns out to be independent of $n$, and defines commutative, associative products $\odot$ on $K_{0}^{\hat{\mu}}\left(\operatorname{Sch}_{X}\right)$ and $\mathcal{M}_{X}^{\hat{\mu}}$.

Let $X, Y$ be $\mathbb{K}$-schemes. As for Definitions 5.1 and 5.2, we define products

$$
\bullet: K_{0}^{\hat{\mu}}\left(\operatorname{Sch}_{X}\right) \times K_{0}^{\hat{\mu}}\left(\operatorname{Sch}_{Y}\right) \rightarrow K_{0}^{\hat{\mu}}\left(\operatorname{Sch}_{X \times Y}\right), \bullet: \mathcal{M}_{X}^{\hat{\mu}} \times \mathcal{M}_{X}^{\hat{\mu}} \rightarrow \mathcal{M}_{X \times Y}^{\hat{\mu}}
$$

by following the definition above for $\odot$, but taking products $R \times S$ rather than fibre products $R \times_{X} S$. These $\square$ are commutative and associative. Taking $Y=$ Spec $\mathbb{K}$, we see that $\square$ makes $K_{0}^{\hat{\mu}}\left(\operatorname{Sch}_{X}\right), \mathcal{M}_{X}^{\hat{\mu}}$ into modules over $K_{0}^{\hat{\mu}}\left(\operatorname{Sch}_{\mathbb{K}}\right), \mathcal{M}_{\mathbb{K}}^{\hat{\mu}}$.

For generators $[R, \rho, \hat{r}]$ and $[S, \sigma, \hat{\imath}]=i_{X}([S, \sigma])$ in $K_{0}^{\hat{\mu}}\left(\operatorname{Sch}_{X}\right)$ or $\mathcal{M}_{X}^{\hat{\mu}}$ where $[S, \sigma, \hat{\iota}]$ has trivial $\hat{\mu}$-action $\hat{\iota}$, one can show that $[R, \rho, \hat{r}] \odot[S, \sigma, \hat{\iota}]=[R, \rho, \hat{r}]$. $[S, \sigma, \hat{\imath}]$. Thus $i_{X}$ is a ring morphism $\left(K_{0}\left(\operatorname{Sch}_{X}\right), \cdot\right) \rightarrow\left(K_{0}^{\hat{\mu}}\left(\operatorname{Sch}_{X}\right), \odot\right)$ and $\left(\mathcal{M}_{X}, \cdot\right) \rightarrow\left(\mathcal{M}_{X}^{\hat{\mu}}, \odot\right)$. However, $\Pi_{X}$ is not a ring morphism $\left(K_{0}^{\hat{\mu}}\left(\operatorname{Sch}_{X}\right), \odot\right) \rightarrow$ $\left(K_{0}\left(\operatorname{Sch}_{X}\right), \cdot\right)$ or $\left(\mathcal{M}_{X}^{\hat{\mu}}, \odot\right) \rightarrow\left(\mathcal{M}_{X}, \cdot\right)$. Since $\mathbb{L}=\left[\mathbb{A}^{1} \times X, \pi_{X}, \hat{\iota}\right]$ this implies that $M \cdot \mathbb{L}=M \odot \mathbb{L}$ for all $M$ in $K_{0}^{\hat{\mu}}\left(\operatorname{Sch}_{X}\right), \mathcal{M}_{X}^{\hat{\mu}}$.

Definition 5.4. Define the element $\mathbb{L}^{1 / 2}$ in $K_{0}^{\hat{\mu}}\left(\operatorname{Sch}_{X}\right)$ and $\mathcal{M}_{X}^{\hat{\mu}}$ by

$$
\mathbb{L}^{1 / 2}=\left[X, \operatorname{id}_{X}, \hat{\iota}\right]-\left[X \times \mu_{2}, \hat{r}\right],
$$

where $\left[X, \operatorname{id}_{X}, \hat{\iota}\right]$ with trivial $\hat{\mu}$-action $\hat{\iota}$ is the identity $1_{X}$ in $K_{0}^{\hat{\mu}}\left(\operatorname{Sch}_{X}\right), \mathcal{M}_{X}^{\hat{\mu}}$, and $X \times \mu_{2}=X \times\{1,-1\}$ is two copies of $X$ with nontrivial $\hat{\mu}$-action $\hat{r}$ induced by the left action of $\mu_{2}$ on itself, exchanging the two copies of $X$. Applying (5.11) with $n=2$, we can show that $\mathbb{L}^{1 / 2} \odot \mathbb{L}^{1 / 2}=\mathbb{L}$. Thus, $\mathbb{L}^{1 / 2}$ in $(5.12)$ is a square root for $\mathbb{L}$ in the rings $\left(K_{0}^{\hat{\mu}}\left(\operatorname{Sch}_{X}\right), \odot\right),\left(\mathcal{M}_{X}^{\hat{\mu}}, \odot\right)$. Note that $\mathbb{L}^{1 / 2} \cdot \mathbb{L}^{1 / 2} \neq \mathbb{L}$.

Equivalently, we could have defined

$$
\mathbb{L}_{X}^{1 / 2}=\left[X, \operatorname{id}_{X}, \hat{\imath}\right] \sqcup \mathbb{L}_{\mathbb{K}}^{1 / 2} \in K_{0}^{\hat{\mu}}\left(\operatorname{Sch}_{X}\right),
$$

where $\mathbb{L}_{\mathbb{K}}^{1 / 2} \in K_{0}^{\hat{\mu}}\left(\mathrm{Sch}_{\mathbb{K}}\right)$. We can now define $\mathbb{L}^{n / 2} \in K_{0}^{\hat{\mu}}\left(\mathrm{Sch}_{X}\right)$ for $n \geqslant 0$ and $\mathbb{L}^{n / 2} \in \mathcal{M}_{X}^{\hat{\mu}}$ for $n \in \mathbb{Z}$ in the obvious way, such that $\mathbb{L}^{m / 2} \odot \mathbb{L}^{n / 2}=\mathbb{L}^{(m+n) / 2}$. 
Next, following [5, §2.5], which was motivated by ideas in Kontsevich and Soibelman [18, §4.5], we define principal $\mathbb{Z} / 2 \mathbb{Z}$-bundles $P \rightarrow X$, associated motives $\Upsilon(P)$, and a quotient ring of motives $\overline{\mathcal{M}}_{X}^{\hat{\mu}}$ in which $\Upsilon\left(P \otimes_{\mathbb{Z} / 2 \mathbb{Z}} Q\right)=$ $\Upsilon(P) \odot \Upsilon(Q)$ for all $P, Q$.

Definition 5.5. Let $X$ be a $\mathbb{K}$-scheme. A principal $\mathbb{Z} / 2 \mathbb{Z}$-bundle $P \rightarrow X$ is a proper, surjective, étale morphism of $\mathbb{K}$-schemes $\pi: P \rightarrow X$ together with a free involution $\sigma: P \rightarrow P$, such that the orbits of $\mathbb{Z} / 2 \mathbb{Z}=\{1, \sigma\}$ are the fibres of $\pi$. The trivial $\mathbb{Z} / 2 \mathbb{Z}$-bundle is $\pi_{X}: X \times \mathbb{Z} / 2 \mathbb{Z} \rightarrow X$. We will use the ideas of isomorphism of principal bundles $\iota: P \rightarrow Q$, section $s: X \rightarrow P$, tensor product $P \otimes_{\mathbb{Z} / 2 \mathbb{Z}} Q$, and pullback $f^{*}(P) \rightarrow Y$ under a 1-morphism of stacks $f: Y \rightarrow X$, all of which are defined in the obvious ways.

Write $(\mathbb{Z} / 2 \mathbb{Z})(X)$ for the abelian group of isomorphism classes $[P]$ of principal $\mathbb{Z} / 2 \mathbb{Z}$-bundles $P \rightarrow X$, with multiplication $[P] \cdot[Q]=\left[P \otimes_{\mathbb{Z} / 2 \mathbb{Z}} Q\right]$ and identity $[X \times \mathbb{Z} / 2 \mathbb{Z}]$. Since $P \otimes_{\mathbb{Z} / 2 \mathbb{Z}} P \cong X \times \mathbb{Z} / 2 \mathbb{Z}$ for each $P \rightarrow X$, each element of $(\mathbb{Z} / 2 \mathbb{Z})(X)$ is self-inverse, and has order 1 or 2 .

If $\pi: P \rightarrow X$ is a principal $\mathbb{Z} / 2 \mathbb{Z}$-bundle over $X$, define a motive

$$
\Upsilon(P)=\mathbb{L}^{-1 / 2} \odot([X, \mathrm{id}, \hat{\imath}]-[P, \pi, \hat{r}]) \in \mathcal{M}_{X}^{\hat{\mu}},
$$

where $\hat{r}$ is the $\hat{\mu}$-action on $P$ induced by the $\mu_{2}$-action on $P$ from the principal $\mathbb{Z} / 2 \mathbb{Z}$-bundle structure, as $\mu_{2} \cong \mathbb{Z} / 2 \mathbb{Z}$. If $P=X \times \mathbb{Z} / 2 \mathbb{Z}$ is trivial then

$$
\begin{aligned}
\Upsilon(X \times \mathbb{Z} / 2 \mathbb{Z}) & =\mathbb{L}^{-1 / 2} \odot([X, \mathrm{id}, \hat{\iota}]-[X \times \mathbb{Z} / 2 \mathbb{Z}, \pi, \hat{r}]) \\
& =\mathbb{L}^{-1 / 2} \odot \mathbb{L}^{1 / 2} \odot[X, \mathrm{id}, \hat{\iota}]=[X, \mathrm{id}, \hat{\iota}]
\end{aligned}
$$

using $(5.12)$. Note that $[X, \mathrm{id}, \hat{\imath}]$ is the identity in the $\operatorname{ring} \mathcal{M}_{X}^{\hat{\mu}}$.

As $\Upsilon(P)$ only depends on $P$ up to isomorphism, $\Upsilon$ factors via $(\mathbb{Z} / 2 \mathbb{Z})(X)$, and we may consider $\Upsilon$ as a map $(\mathbb{Z} / 2 \mathbb{Z})(X) \rightarrow \mathcal{M}_{X}^{\hat{\mu}}$.

For our applications, we want $\Upsilon:(\mathbb{Z} / 2 \mathbb{Z})(X) \rightarrow \mathcal{M}_{X}^{\hat{\mu}}$ to be a group morphism with respect to the multiplication $\odot$ on $\mathcal{M}_{X}^{\hat{\mu}}$, but we cannot prove that it is. Our solution is to pass to a quotient ring $\overline{\mathcal{M}}_{X}^{\hat{\mu}}$ of $\mathcal{M}_{X}^{\hat{\mu}}$ such that the induced map $\Upsilon:(\mathbb{Z} / 2 \mathbb{Z})(X) \rightarrow \overline{\mathcal{M}}_{X}^{\hat{\mu}}$ is a group morphism. If we simply defined $\overline{\mathcal{M}}_{X}^{\hat{\mu}}$ to be the quotient ring of $\mathcal{M}_{X}^{\hat{\mu}}$ by the relations $\Upsilon\left(P \otimes_{\mathbb{Z} / 2 \mathbb{Z}} Q\right)-\Upsilon(P) \odot \Upsilon(Q)=0$ for all $[P],[Q]$ in $(\mathbb{Z} / 2 \mathbb{Z})(X)$ then pushforwards $\phi_{*}: \overline{\mathcal{M}}_{X}^{\hat{\mu}} \rightarrow \overline{\mathcal{M}}_{Y}^{\hat{\mu}}$ would not be defined for general $\phi: X \rightarrow Y$. So we impose a more complicated relation.

For each $\mathbb{K}$-scheme $Y$, define $I_{Y}^{\hat{\mu}}$ to be the ideal in the commutative ring $\left(\mathcal{M}_{Y}^{\hat{\mu}}, \odot\right)$ generated by elements $\phi_{*}\left(\Upsilon\left(P \otimes_{\mathbb{Z} / 2 \mathbb{Z}} Q\right)-\Upsilon(P) \odot \Upsilon(Q)\right)$ for all $\mathbb{K}$ scheme morphisms $\phi: X \rightarrow Y$ and principal $\mathbb{Z} / 2 \mathbb{Z}$-bundles $P, Q \rightarrow X$, and define $\overline{\mathcal{M}}_{Y}^{\hat{\mu}}=\mathcal{M}_{Y}^{\hat{\mu}} / I_{Y}^{\hat{\mu}}$ to be the quotient, as a commutative ring with multiplication ' $\odot$ ', with projection $\Pi_{Y}^{\hat{\mu}}: \mathcal{M}_{Y}^{\hat{\mu}} \rightarrow \overline{\mathcal{M}}_{Y}^{\hat{\mu}}$. Kontsevich and Soibelman [18, §4.5] introduce a relation in their motivic rings which has a similar effect.

Note that in $\overline{\mathcal{M}}_{Y}^{\hat{\mu}}$ we do not have the second multiplication '.', since we do not require $I_{Y}^{\hat{\mu}}$ to be an ideal in $\left(\mathcal{M}_{Y}^{\hat{\mu}}, \cdot\right)$. Also $\otimes$ and $\Pi_{Y}: \mathcal{M}_{Y}^{\hat{\mu}} \rightarrow \mathcal{M}_{Y}$ on $\mathcal{M}_{Y}^{\hat{\mu}}$ do not descend to $\overline{\mathcal{M}}_{Y}^{\hat{\mu}}$. Apart from this, all the structures on $\mathcal{M}_{Y}^{\hat{\mu}}$ 
above descend to $\overline{\mathcal{M}}_{Y}^{\hat{\mu}}$ : operations $\odot, \odot$, pushforwards $\phi_{*}$ and pullbacks $\phi^{*}$, and elements $\mathbb{L}, \mathbb{L}^{1 / 2}, \Upsilon(P)$. By definition, $\overline{\mathcal{M}}_{X}^{\hat{\mu}}$ has the property that

$$
\Upsilon\left(P \otimes_{\mathbb{Z} / 2 \mathbb{Z}} Q\right)=\Upsilon(P) \odot \Upsilon(Q) \quad \text { in } \overline{\mathcal{M}}_{X}^{\hat{\mu}}
$$

for all principal $\mathbb{Z} / 2 \mathbb{Z}$-bundles $P, Q \rightarrow X$.

\subsection{Motivic vanishing cycles, and d-critical loci}

Following Denef and Loeser [6], we define motivic nearby cycles, motivic Milnor fibres, and motivic vanishing cycles:

Definition 5.6. Let $U$ be a smooth $\mathbb{K}$-scheme and $f: U \rightarrow \mathbb{A}^{1}$ a regular function, and set $U_{0}=f^{-1}(0) \subseteq U$. Then Denef and Loeser [6, §3.5] and Looijenga [27, §5] define the motivic nearby cycle of $f$, an element $M F_{U, f}^{\operatorname{mot}}$ of $\mathcal{M}_{U_{0}}^{\hat{\mu}}$ or $\overline{\mathcal{M}}_{U_{0}}^{\hat{\mu}}$. It has an intrinsic definition using arc spaces and the motivic zeta function, which we will not explain, but we will give a formula [6, §3.3], 27, §5] for $M F_{U, f}^{\text {mot }}$ involving choosing a resolution of $f$.

If $f=0$ then $M F_{U, f}^{\text {mot }}=0$, so suppose $f$ is not constant. By Hironaka's Theorem [13] we can choose a resolution $(\tilde{U}, \pi)$ of $f$. That is, $\tilde{U}$ is a smooth $\mathbb{K}$-scheme and $\pi: \tilde{U} \rightarrow U$ a proper morphism, such that $\left.\pi\right|_{\tilde{U} \backslash \pi^{-1}\left(U_{0}\right)}: \tilde{U} \backslash$ $\pi^{-1}\left(U_{0}\right) \rightarrow U \backslash U_{0}$ is an isomorphism, and $\pi^{-1}\left(U_{0}\right)^{\text {red }}$ has only normal crossings as a $\mathbb{K}$-subscheme of $\tilde{U}$.

Write $E_{i}, i \in J$ for the irreducible components of $\pi^{-1}\left(U_{0}\right)$. For each $i \in J$, denote by $N_{i}$ the multiplicity of $E_{i}$ in the divisor of $f \circ \pi$ on $\tilde{U}$, and by $\nu_{i}-1$ the multiplicity of $E_{i}$ in the divisor of $\pi^{*}(\mathrm{~d} x)$, where $\mathrm{d} x$ is a local non vanishing volume form at any point of $\pi\left(E_{i}\right)$. For $I \subset J$, we consider the smooth $\mathbb{K}$ scheme $E_{I}^{\circ}=\left(\bigcap_{i \in I} E_{i}\right) \backslash\left(\bigcup_{j \in J \backslash I} E_{j}\right)$.

Let $m_{I}=\operatorname{gcd}\left(N_{i}\right)_{i \in I}$. We introduce an unramified Galois cover $\tilde{E}_{I}^{\circ}$ of $E_{I}^{\circ}$, with Galois group $\mu_{m_{I}}$, as follows. Let $\tilde{U}^{\prime}$ be an affine Zariski open subset of $\tilde{U}$, such that, on $\tilde{U}^{\prime}, f \circ \pi=u v^{m_{I}}$, with $u: \tilde{U}^{\prime} \rightarrow \mathbb{A}^{1} \backslash\{0\}$ and $v: \tilde{U}^{\prime} \rightarrow \mathbb{A}^{1}$. Then the restriction of $\tilde{E}_{I}^{\circ}$ above $E_{I}^{\circ} \cap \tilde{U}^{\prime}$, denoted by $\tilde{E}_{I}^{\circ} \cap \tilde{U}^{\prime}$, is defined as

$$
\tilde{E}_{I}^{\circ} \cap \tilde{U}^{\prime}=\left\{(z, w) \in \mathbb{A}^{1} \times\left(E_{I}^{\circ} \cap \tilde{U}^{\prime}\right): z^{m_{I}}=u(w)^{-1}\right\} .
$$

Gluing together the covers $\tilde{E}_{I}^{\circ} \cap \tilde{U}^{\prime}$ in the obvious way, we obtain the cover $\tilde{E}_{I}^{\circ}$ of $E_{I}^{\circ}$ which has a natural $\mu_{m_{I}}$-action $\rho_{I}$, obtained by multiplying the $z$-coordinate by elements of $\mu_{m_{I}}$. This $\mu_{m_{I}}$-action on $\tilde{E}_{I}^{\circ}$ induces a $\hat{\mu}$-action $\hat{\rho}_{I}$ on $\tilde{E}_{I}^{\circ}$. Then

$$
M F_{U, f}^{\operatorname{mot}}=\sum_{\emptyset \neq I \subseteq J}(1-\mathbb{L})^{|I|-1}\left[\tilde{E}_{I}^{\circ}, \pi_{U_{0}}, \hat{\rho}_{I}\right] \quad \text { in } \mathcal{M}_{U_{0}}^{\hat{\mu}} .
$$

It is independent of the choice of resolution $(\tilde{U}, \pi)$. The fibre $\left.M F_{U, f}^{\operatorname{mot}}\right|_{x}$ at each $x \in U_{0}$ is called the motivic Milnor fibre of $f$ at $x$.

Now let $X=\operatorname{Crit}(f) \subseteq U$, as a closed $\mathbb{K}$-subscheme of $U$. Since $f$ is constant on the reduced scheme $X^{\text {red }}, f(X)$ is finite, and we may write $X=\coprod_{c \in f(X)} X_{c}$, where $X_{c} \subseteq X$ is the open and closed $\mathbb{K}$-subscheme with $X_{c}^{\text {red }}=\left.f\right|_{X^{\text {red }}} ^{-1}(c)$. 
Consider the restriction $\left.M F_{U, f}^{\text {mot }}\right|_{U_{0} \backslash X_{0}}$ in $\mathcal{M}_{U_{0} \backslash X_{0}}^{\hat{\hat{}}}$ or $\overline{\mathcal{M}}_{U_{0} \backslash X_{0}}^{\hat{\mu}}$. We can choose $(\tilde{U}, \pi)$ above with $\left.\pi\right|_{\tilde{U} \backslash \pi^{-1}\left(X_{0}\right)}: \tilde{U} \backslash \pi^{-1}\left(X_{0}\right) \rightarrow U \backslash X_{0}$ an isomorphism. Write $D_{1}, \ldots, D_{k}$ for the irreducible components of $\pi^{-1}\left(U_{0} \backslash X_{0}\right) \cong U_{0} \backslash X_{0}$. They are disjoint as $\pi^{-1}\left(U_{0} \backslash X_{0}\right)$ is nonsingular. The closures $\bar{D}_{1}, \ldots, \bar{D}_{k}$ (which need not be disjoint) are among the divisors $E_{i}$, so we write $\bar{D}_{a}=E_{i_{a}}$ for $a=1, \ldots, k$, with $\left\{i_{1}, \ldots, i_{k}\right\} \subseteq I$. Clearly $N_{i_{a}}=\nu_{i_{a}}=1$ for $a=1, \ldots, k$.

Then in (5.14) the only nonzero contributions to $\left.M F_{U, f}^{\operatorname{mot}}\right|_{U_{0} \backslash X_{0}}$ are from $I=\left\{i_{a}\right\}$ for $a=1, \ldots, k$, with $\tilde{E}_{\left\{i_{a}\right\}}^{\circ} \cong E_{\left\{i_{a}\right\}}^{\circ} \cong D_{a}$, and the $\hat{\mu}$-action on $\tilde{E}_{\left\{i_{a}\right\}}^{\circ}$ is trivial as it factors through the action of $\mu_{1}=\{1\}$. Hence

$$
\left.M F_{U, f}^{\mathrm{mot}}\right|_{U_{0} \backslash X_{0}}=\sum_{a=1}^{k}\left[\tilde{E}_{\left\{i_{a}\right\}}^{\circ}, \pi_{U_{0} \backslash X_{0}}, \hat{\iota}\right]=\sum_{a=1}^{k}\left[D_{a}, \pi_{U_{0} \backslash X_{0}}, \hat{\iota}\right]=\left[U_{0} \backslash X_{0}, \operatorname{id}_{U_{0} \backslash X_{0}}, \hat{\iota}\right] .
$$

Therefore $\left[U_{0}, \operatorname{id}_{U_{0}}, \hat{\imath}\right]-M F_{U, f}^{\text {mot }}$ is supported on $X_{0} \subseteq U_{0}$, and by restricting to $X_{0}$ we regard it as an element of $\mathcal{M}_{X_{0}}^{\hat{\mu}}$ or $\overline{\mathcal{M}}_{X_{0}}^{\hat{\mu}}$.

Define the motivic vanishing cycle $M F_{U, f}^{\text {mot, } \phi}$ of $f$ in $\mathcal{M}_{X}^{\hat{\mu}}$ or $\overline{\mathcal{M}}_{X}^{\hat{\mu}}$ by

$$
\left.M F_{U, f}^{\operatorname{mot}, \phi}\right|_{X_{c}}=\left.\mathbb{L}^{-\operatorname{dim} U / 2} \odot\left(\left[U_{c}, \operatorname{id}_{U_{c}}, \hat{\imath}\right]-M F_{U, f-c}^{\operatorname{mot}}\right)\right|_{X_{c}}
$$

for each $c \in f(X)$, where $\odot$ and $\mathbb{L}^{-\operatorname{dim} U / 2}$ are as in Definitions 5.3 and 5.4

Here is [5, Th. 5.10], which we will generalize to stacks in Theorem 5.14.

Theorem 5.7. Let $(X, s)$ be an algebraic d-critical locus with orientation $K_{X, s}^{1 / 2}$, for $X$ of finite type. Then there exists a unique motive $M F_{X, s} \in \overline{\mathcal{M}}_{X}^{\hat{\mu}}$ with the property that if $(R, U, f, i)$ is a critical chart on $(X, s)$, then

$$
\left.M F_{X, s}\right|_{R}=i^{*}\left(M F_{U, f}^{\mathrm{mot}, \phi}\right) \odot \Upsilon\left(Q_{R, U, f, i}\right) \text { in } \overline{\mathcal{M}}_{R}^{\hat{\mu}},
$$

where $Q_{R, U, f, i} \rightarrow R$ is the principal $\mathbb{Z} / 2 \mathbb{Z}$-bundle parametrizing local isomorphisms $\alpha:\left.\left.K_{X, s}^{1 / 2}\right|_{R^{\mathrm{red}}} \rightarrow i^{*}\left(K_{U}\right)\right|_{R^{\mathrm{red}}}$ with $\alpha \otimes \alpha=\iota_{R, U, f, i}$, for $\iota_{R, U, f, i}$ as in (3.4).

We prove a result on smooth pullbacks and pushforwards of the motives $M F_{X, s}$ of Theorem 5.7, a motivic analogue of Proposition 4.5(a).

Proposition 5.8. Let $\phi:(X, s) \rightarrow(Y, t)$ be a morphism of (finite type) algebraic d-critical loci in the sense of 33.1, and suppose $\phi: X \rightarrow Y$ is smooth of relative dimension $n$. Let $K_{Y, t}^{1 / 2}$ be an orientation for $(Y, t)$, so that Corollary 3.8 defines an induced orientation $K_{X, s}^{1 / 2}$ for $(X, s)$. Theorem 5.7 now defines motives $M F_{X, s}, M F_{Y, t}$ on $X, Y$. These are related by

$$
\begin{aligned}
\phi^{*}\left(M F_{Y, t}\right) & =\mathbb{L}^{n / 2} \odot M F_{X, s} \in \overline{\mathcal{M}}_{X}^{\hat{\mu}}, \\
\phi_{*}\left(M F_{X, s}\right) & =\mathbb{L}^{-n / 2} \odot M F_{Y, t} \odot[X, \phi, \hat{\iota}] \in \overline{\mathcal{M}}_{Y}^{\hat{\mu}} .
\end{aligned}
$$


Proof. If $x \in X$ with $\phi(x)=y \in Y$ then the proof of Proposition 3.2 above in [15] shows we may choose critical charts $(R, U, f, i),(S, V, g, j)$ on $(X, s),(Y, t)$ with $x \in R, y \in \phi(R) \subseteq S$ of minimal dimensions $\operatorname{dim} U=\operatorname{dim} T_{x} X, \operatorname{dim} V=$ $\operatorname{dim} T_{y} Y$, and $\Phi: U \rightarrow V$ smooth of relative dimension $n$ with $f=g \circ \Phi$ and $\Phi \circ i=j \circ \phi$.

Let $\pi: \tilde{V} \rightarrow V$ be an embedded resolution of singularities of $g$. Then $\tilde{U}:=$ $U \times_{\Phi, V, \pi} \tilde{V}$ is an embedded resolution of singularities of $f$, since $\Phi$ is smooth and $f=g \circ \Phi$. As in Definition 5.6, let $F_{i}$ for $i \in J$ be the irreducible components of $\pi^{-1}\left(V_{0}\right)$, so that $\pi^{-1}\left(V_{0}\right)=\bigcup_{i \in J} F_{i}$, with multiplicities $N_{i}$ in the divisor of $g \circ \pi$ on $\tilde{V}$, and $\nu_{i}-1$ in the divisor of $\pi^{*}(\mathrm{~d} x)$, and define $F_{I}^{\circ}=\left(\bigcap_{i \in I} F_{i}\right) \backslash\left(\bigcup_{j \in J \backslash I} F_{j}\right)$ and covers $\tilde{F}_{I}^{\circ} \rightarrow F_{I}^{\circ}$ for all $I \subseteq J$.

Define $E_{i}=U \times_{\Phi, V,\left.\pi\right|_{F_{i}}} F_{i} \subset \pi^{-1}\left(U_{0}\right) \subset \tilde{U}$. Then $\pi^{-1}\left(U_{0}\right)=\bigcup_{i \in J} E_{i}$. The $E_{i}$ need not be irreducible, or nonempty, but this is not important. Neglecting this, we can treat the $E_{i}, i \in J$ as the components for $(\tilde{U}, \pi)$ in Definition 5.6. and then they have the same multiplicities $N_{i}, \nu_{i}$ as the $F_{i}$ for $(\tilde{V}, \pi)$, and the $E_{I}^{\circ}, \tilde{E}_{I}^{\circ}$ for $I \subseteq J$ defined in Definition [5.6] satisfy $E_{I}^{\circ} \cong U \times_{V} F_{I}^{\circ}$ and $\tilde{E}_{I}^{\circ} \cong U \times_{V} \tilde{F}_{I}^{\circ}$. Thus we have

$$
\begin{aligned}
M F_{U, f}^{\operatorname{mot}} & =\sum_{\emptyset \neq I \subseteq J}(1-\mathbb{L})^{|I|-1}\left[\tilde{E}_{I}^{\circ}, \pi_{U_{0}}, \hat{\rho}_{I}\right] \\
& =\sum_{\emptyset \neq I \subseteq J}(1-\mathbb{L})^{|I|-1}\left[\tilde{F}_{I}^{\circ} \times_{\pi_{V_{0}}, V_{0},\left.\Phi\right|_{U_{0}}} U_{0}, \pi_{U_{0}}, \hat{\rho}_{I}\right] \\
& =\left.\Phi\right|_{U_{0}} ^{*}\left[\sum_{\emptyset \neq I \subseteq J}(1-\mathbb{L})^{|I|-1}\left[\tilde{F}_{I}^{\circ}, \pi_{V_{0}}, \hat{\rho}_{I}\right]\right]=\left.\Phi\right|_{U_{0}} ^{*}\left(M F_{V, g}^{\mathrm{mot}}\right) .
\end{aligned}
$$

So from (5.15) we deduce that

$$
\left.\Phi\right|_{\operatorname{Crit}(f)} ^{*}\left(M F_{V, g}^{\mathrm{mot}, \phi}\right)=\mathbb{L}^{n / 2} \odot M F_{U, f}^{\mathrm{mot}, \phi},
$$

using $\left.\Phi\right|_{U_{c}} ^{*}\left(\left[V_{c}, \operatorname{id}_{V_{c}}, \hat{\imath}\right]\right)=\left[U_{c}, \mathrm{id}_{U_{c}}, \hat{\imath}\right]$, where the factor $\mathbb{L}^{n / 2}$ is to convert the factor $\mathbb{L}^{-\operatorname{dim} U / 2}$ in $M F_{U, f}^{\operatorname{mot}, \phi}$ to the factor $\mathbb{L}^{-\operatorname{dim} V / 2}$ in $M F_{V, g}^{\operatorname{mot}, \phi}$.

Combining (5.19) with (5.16) for $(X, s),(R, U, f, i)$ and the pullback of (5.16) for $(Y, t),(S, V, g, j)$ by $\left.\phi\right|_{R}: R \rightarrow S$, and noting that $\phi^{*} \circ j^{*}=\left.i^{*} \circ \Phi\right|_{\operatorname{Crit}(f)} ^{*}$ since $j \circ \phi=\Phi \circ i$, we deduce the restriction of (5.17) to $R \subseteq X$. As we can cover $X$ by such open $R$, this proves (5.17). Equation (5.18) follows by applying $\phi_{*}$ and noting that $\phi_{*} \circ \phi^{*}(M)=M \odot[X, \phi, \hat{\imath}]$ for all $\phi: X \rightarrow Y$ and $M \in \overline{\mathcal{M}}_{Y}^{\hat{\mu}}$.

\subsection{Rings of motives over Artin stacks}

We now generalize the material of $\$ 5.1$ to Artin stacks. Our definitions are new, but very similar to work by Joyce [14 on 'stack functions', and Kontsevich and Soibelman [19, §4.1-§4.2]. As in [14, we restrict our attention to Artin $\mathbb{K}$-stacks $X$ (always assumed of finite type) with affine geometric stabilizers. In $\$ 5.4-55.5$ we will restrict further, to stacks which are locally a global quotient.

Definition 5.9. An Artin $\mathbb{K}$-stack $X$ has affine geometric stabilizers if the stabilizer group $\operatorname{Iso}_{X}(x)$ is an affine algebraic group for all points $x \in X$. 
An Artin $\mathbb{K}$-stack $X$ is locally a global quotient if we may cover $X$ by Zariski open $\mathbb{K}$-substacks $Y \subseteq X$ equivalent to global quotients $[S / \mathrm{GL}(n, \mathbb{K})]$, where $S$ is a $\mathbb{K}$-scheme with a $\mathrm{GL}(n, \mathbb{K})$-action.

If $X$ is locally a global quotient then it has affine geometric stabilizers, since the stabilizer groups of $[S / \mathrm{GL}(n, \mathbb{K})]$ are closed $\mathbb{K}$-subgroups of $\mathrm{GL}(n, \mathbb{K})$, and so are affine. The authors do not know any example of an Artin $\mathbb{K}$-stack with affine geometric stabilizers which is not locally a global quotient.

Deligne-Mumford stacks have affine geometric stabilizers, and are locally a global quotient if their stabilizers are generically trivial. If $\mathcal{M}$ is a moduli stack of coherent sheaves $F$ on a projective scheme $Y$, then using Quot-schemes one can show that $\mathcal{M}$ is locally a global quotient. If $\mathcal{M}$ is a moduli stack of complexes $F^{\bullet}$ in $D^{b} \operatorname{coh}(Y)$ with $\operatorname{Ext}^{<0}\left(F^{\bullet}, F^{\bullet}\right)=0$ then $\mathcal{M}$ has affine geometric stabilizers, since $\operatorname{Iso}_{\mathcal{M}}\left(F^{\bullet}\right)$ is the invertible elements in the finite-dimensional algebra $\operatorname{Hom}\left(F^{\bullet}, F^{\bullet}\right)$, and so is affine. We require affine geometric stabilizers to use a result of Kresch [20, Prop. 3.5.9]:

Proposition 5.10 (Kresch). Let $X$ be a (finite type) Artin $\mathbb{K}$-stack with affine geometric stabilizers. Then $X$ admits a stratification $X=\coprod_{i \in I} X_{i}$, for $I$ a finite set and $X_{i} \subseteq X$ a locally closed $\mathbb{K}$-substack, such that $X_{i}$ is equivalent to a global quotient stack $\left[S_{i} / \mathrm{GL}\left(n_{i}, \mathbb{K}\right)\right]$ for each $i \in I$, where $S_{i}$ is a (finite type) $\mathbb{K}$-scheme with an action of $\mathrm{GL}\left(n_{i}, \mathbb{K}\right)$. Conversely, any Artin $\mathbb{K}$-stack $X$ admitting such a stratification has affine geometric stabilizers.

For the rest of this paper, all Artin $\mathbb{K}$-stacks $X$ are assumed to have affine geometric stabilizers. Here are the analogues of Definitions 5.1 and 5.2

Definition 5.11. Let $X$ be an Artin $\mathbb{K}$-stack (always assumed to be of finite type, with affine geometric stabilizers). Consider pairs $(R, \rho)$, where $R$ is a $\mathbb{K}$ scheme and $\rho: R \rightarrow X$ a 1-morphism. Call two pairs $(R, \rho),\left(R^{\prime}, \rho^{\prime}\right)$ equivalent if there exists an isomorphism $\iota: R \rightarrow R^{\prime}$ such that $\rho^{\prime} \circ \iota$ and $\rho$ are 2 -isomorphic 1 -morphisms $R \rightarrow X$. Write $[R, \rho]$ for the equivalence class of $(R, \rho)$. Define the Grothendieck ring $K_{0}\left(\operatorname{Sch}_{X}\right)$ of the category of $\mathbb{K}$-schemes over $X$ to be the abelian group generated by equivalence classes $[R, \rho]$, such that as for (5.1) for each closed $\mathbb{K}$-subscheme $S$ of $R$ we have

$$
[R, \rho]=\left[S,\left.\rho\right|_{S}\right]+\left[R \backslash S,\left.\rho\right|_{R \backslash S}\right] .
$$

When $X=\operatorname{Spec} \mathbb{K}$ we write $K_{0}\left(\mathrm{Sch}_{\mathbb{K}}\right)$ instead of $K_{0}\left(\mathrm{Sch}_{X}\right)$.

Define a biadditive, commutative, associative product '. ' on $K_{0}\left(\mathrm{Sch}_{X}\right)$ as in (5.2). It makes $K_{0}\left(\operatorname{Sch}_{X}\right)$ into a commutative ring, in general without identity. If $X$ is a $\mathbb{K}$-scheme $K_{0}\left(\operatorname{Sch}_{X}\right)$ is as in Definition [5.1, with identity $\left[X, \mathrm{id}_{X}\right]$.

For Artin $\mathbb{K}$-stacks $X, Y$, define a biadditive, commutative, associative external tensor product $\square: K_{0}\left(\operatorname{Sch}_{X}\right) \times K_{0}\left(\operatorname{Sch}_{Y}\right) \rightarrow K_{0}\left(\operatorname{Sch}_{X \times Y}\right)$ by (5.4). Taking $Y=\operatorname{Spec} \mathbb{K}$ we see that $\otimes$ makes $K_{0}\left(\operatorname{Sch}_{X}\right)$ into a module over $K_{0}\left(\mathrm{Sch}_{\mathbb{K}}\right)$.

Next we will define a stack analogue $\mathcal{M}_{X}^{\text {st }}$ of the motivic ring $\mathcal{M}_{X}$ of (5.3) for $\mathbb{K}$-schemes $X$. Since we have no identity in $K_{0}\left(\operatorname{Sch}_{X}\right)$ if $X$ is not a scheme, and we have not defined a Tate motive $\mathbb{L}$ in $K_{0}\left(\mathrm{Sch}_{X}\right)$, the analogue of (5.3) does not make sense. Instead, we use the $K_{0}\left(\mathrm{Sch}_{\mathbb{K}}\right)$-module structure, and define

$$
\mathcal{M}_{X}^{\text {st }}=K_{0}\left(\operatorname{Sch}_{X}\right) \otimes_{K_{0}\left(\operatorname{Sch}_{\mathbb{K}}\right)} K_{0}\left(\mathrm{Sch}_{\mathbb{K}}\right)\left[\mathbb{L}^{-1},\left(\mathbb{L}^{k}-1\right)^{-1}, k=1,2, \ldots\right],
$$


where $\mathbb{L} \in K_{0}\left(\mathrm{Sch}_{\mathbb{K}}\right)$ is as in Definition 5.1. The product '.' descends to $\mathcal{M}_{X}^{\text {st }}$. When $X=$ Spec $\mathbb{K}$ we write $\mathcal{M}_{\mathbb{K}}^{\text {st }}$ instead of $\mathcal{M}_{X}^{\text {st }}$.

Note that for $X$ a $\mathbb{K}$-scheme, $\mathcal{M}_{X}^{\text {st }}$ is not isomorphic to $\mathcal{M}_{X}$ in (5.3), since we invert $\mathbb{L}^{k}-1$ in $\mathcal{M}_{X}^{\text {st }}$ but not in $\mathcal{M}_{X}$. There is a natural projection $\mathcal{M}_{X} \rightarrow \mathcal{M}_{X}^{\text {st }}$. The reason we invert $\mathbb{L}^{k}-1$ as well as $\mathbb{L}$ is that the motive of $\operatorname{GL}(n, \mathbb{K})$ in $\mathcal{M}_{\mathbb{K}}$ is

$$
[\operatorname{GL}(n, \mathbb{K})]:=\left[\mathrm{GL}(n, \mathbb{K}), \pi_{\text {Spec } \mathbb{K}}\right]=\mathbb{L}^{n(n-1) / 2} \prod_{k=1}^{n}\left(\mathbb{L}^{k}-1\right),
$$

so that $[\mathrm{GL}(n, \mathbb{K})]$ is invertible in $\mathcal{M}_{\mathbb{K}}^{\text {st }}$.

Let $X$ be an Artin $\mathbb{K}$-stack (as usual of finite type, with affine geometric stabilizers). Then Proposition 5.10 gives a finite stratification $X=\coprod_{i \in I} X_{i}$ with $X_{i} \simeq\left[S_{i} / \mathrm{GL}\left(n_{i}, \mathbb{K}\right)\right]$. Write $\pi_{i}: S_{i} \rightarrow X$ for the composition of 1-morphisms $S_{i} \rightarrow\left[S_{i} / \mathrm{GL}\left(n_{i}, \mathbb{K}\right)\right] \stackrel{\sim}{\longrightarrow} X_{i} \hookrightarrow X$. Define elements $1_{X}, \mathbb{L} \in \mathcal{M}_{X}^{\text {st }}$ by

$$
\begin{aligned}
1_{X} & =\sum_{i \in I}\left[\mathrm{GL}\left(n_{i}, \mathbb{K}\right)\right]^{-1} \otimes\left[S_{i}, \pi_{i}\right], \\
\mathbb{L} & =\sum_{i \in I}\left[\mathrm{GL}\left(n_{i}, \mathbb{K}\right)\right]^{-1} \otimes\left[\mathbb{A}^{1} \times S_{i}, \pi_{i} \circ \pi_{S_{i}}\right],
\end{aligned}
$$

where $\left[\mathrm{GL}\left(n_{i}, \mathbb{K}\right)\right]^{-1} \in \mathcal{M}_{\mathbb{K}}^{\text {st }}$ exists as above. It is easy to show that these $1_{X}, \mathbb{L}$ are independent of the choice of $I, X_{i}, S_{i}, n_{i}$, and $1_{X}$ is the identity in $\left(\mathcal{M}_{X}^{\text {st }}, \cdot\right)$.

Let $\phi: X \rightarrow Y$ be a 1-morphism of Artin $\mathbb{K}$-stacks. Define the pushforwards $\phi_{*}: K_{0}\left(\operatorname{Sch}_{X}\right) \rightarrow K_{0}\left(\operatorname{Sch}_{Y}\right)$ and $\phi_{*}: \mathcal{M}_{X}^{\text {st }} \rightarrow \mathcal{M}_{Y}^{\text {st }}$ by (5.5) . If $\phi$ is representable in $\mathbb{K}$-schemes we may also define pullbacks $\phi^{*}: K_{0}\left(\operatorname{Sch}_{Y}\right) \rightarrow K_{0}\left(\operatorname{Sch}_{X}\right)$ and $\phi^{*}: \mathcal{M}_{Y}^{\mathrm{st}} \rightarrow \mathcal{M}_{X}^{\mathrm{st}}$ by (5.6). (Here $\phi$ is representable in $\mathbb{K}$-schemes if $X \times_{\phi, Y, u} U$ is a $\mathbb{K}$-scheme for all $u: U \rightarrow Y$ with $U$ a $\mathbb{K}$-scheme.) But if $\phi$ is not representable in $\mathbb{K}$-schemes then $R_{i} \times_{\rho_{i}, Y, \phi} X$ in (5.6) may not be a $\mathbb{K}$-scheme, so (5.6) does not make sense.

However, for general 1-morphisms $\phi: X \rightarrow Y$ we can still define a pullback morphism $\phi^{*}: \mathcal{M}_{Y}^{\text {st }} \rightarrow \mathcal{M}_{X}^{\text {st }}$ as follows. Proposition 5.10 gives a finite stratification $X=\coprod_{i \in I} X_{i}$ with $X_{i} \simeq\left[S_{i} / \mathrm{GL}\left(n_{i}, \mathbb{K}\right)\right]$. Let $\pi_{i}: S_{i} \rightarrow X$ be as above, and define a group morphism $\phi^{*}: \mathcal{M}_{Y}^{\text {st }} \rightarrow \mathcal{M}_{X}^{\text {st }}$ by

$$
\phi^{*}: \sum_{j=1}^{n} c_{j}\left[R_{j}, \rho_{j}\right] \longmapsto \sum_{j=1}^{n} c_{j} \sum_{i \in I}\left[\mathrm{GL}\left(n_{i}, \mathbb{K}\right)\right]^{-1} \otimes\left[R_{j} \times_{\rho_{j}, Y, \phi \circ \pi_{i}} S_{i}, \pi_{X}\right] .
$$

If $\phi$ is representable in $\mathbb{K}$-schemes, this is the result of multiplying (5.6) by equation (5.21) for $1_{X}$, and so the two definitions of $\phi^{*}$ agree. As for $1_{X}, \mathbb{L}$ one can show that $\phi^{*}$ is independent of the choice of $I, X_{i}, S_{i}, n_{i}$, and that pullbacks $\phi^{*}$ have the usual functoriality properties. As in [14, Th. 3.5], the analogue of (5.7) holds for 2-Cartesian squares in Artin $\mathbb{K}$-stacks.

Definition 5.12. Let $X$ be an Artin $\mathbb{K}$-stack. Consider triples $(R, \rho, \hat{r})$, where $R$ is a $\mathbb{K}$-scheme, $\rho: R \rightarrow X$ a 1-morphism, and $\hat{r}: \hat{\mu} \times R \rightarrow R$ a good $\hat{\mu}$-action on $R$, in the sense of Definition 5.2. Call two such triples $(R, \rho, \hat{r}),\left(R^{\prime}, \rho^{\prime}, \hat{r}^{\prime}\right)$ equivalent if there exists a $\hat{\mu}$-equivariant isomorphism $\iota: R \rightarrow R^{\prime}$ and a 2 isomorphism $\rho \cong \rho^{\prime} \circ \iota$. Write $[R, \rho, \hat{r}]$ for the equivalence class of $(R, \rho, \hat{r})$.

The monodromic Grothendieck group $K_{0}^{\hat{\mu}}\left(\mathrm{Sch}_{X}\right)$ is the abelian group generated by such equivalence classes $[R, \rho, \hat{r}]$, with relations (i),(ii) as in Definition 5.2. except that we require a 2-isomorphism $\rho_{2} \cong \rho_{1} \circ \pi$ rather than equality $\rho_{2}=\rho_{1} \circ \pi$ in (ii). Define a biadditive, commutative, associative product 
'.' on $K_{0}^{\hat{\mu}}\left(\mathrm{Sch}_{X}\right)$ as in (5.8). As for $K_{0}\left(\mathrm{Sch}_{X}\right)$ in Definition 5.11, this makes $K_{0}^{\hat{\mu}}\left(\operatorname{Sch}_{X}\right)$ into a commutative ring, in general without identity. If $X$ is a $\mathbb{K}$ scheme $K_{0}^{\hat{\mu}}\left(\operatorname{Sch}_{X}\right)$ is as in Definition [5.2, with identity $\left[X, \mathrm{id}_{X}, \hat{\imath}\right]$.

For Artin $\mathbb{K}$-stacks $X, Y$, define a biadditive, commutative, associative external tensor product $\otimes: K_{0}^{\hat{\mu}}\left(\operatorname{Sch}_{X}\right) \times K_{0}^{\hat{\mu}}\left(\operatorname{Sch}_{Y}\right) \rightarrow K_{0}^{\hat{\mu}}\left(\operatorname{Sch}_{X \times Y}\right)$ by (5.9). Taking $Y=\operatorname{Spec} \mathbb{K}$, this makes $K_{0}^{\hat{\mu}}\left(\operatorname{Sch}_{X}\right)$ into a module over $K_{0}^{\hat{\mu}}\left(\operatorname{Sch}_{\mathbb{K}}\right)$.

As for (5.20), using the $K_{0}^{\hat{\mu}}\left(\operatorname{Sch}_{\mathbb{K}}\right)$-module structure on $K_{0}^{\hat{\mu}}\left(\operatorname{Sch}_{X}\right)$ define

$$
\mathcal{M}_{X}^{\text {st, }, \hat{\mu}}=K_{0}^{\hat{\mu}}\left(\operatorname{Sch}_{X}\right) \otimes_{K_{0}^{\hat{\mu}}\left(\operatorname{Sch}_{\mathbb{K}}\right)} K_{0}^{\hat{\mu}}\left(\operatorname{Sch}_{\mathbb{K}}\right)\left[\mathbb{L}^{-1},\left(\mathbb{L}^{k}-1\right)^{-1}, k=1,2, \ldots\right] .
$$

The product '.' descends to $\mathcal{M}_{X}^{\text {st, }, \hat{\mu}}$. When $X=\operatorname{Spec} \mathbb{K}$ we write $\mathcal{M}_{\mathbb{K}}^{\text {st, } \hat{\mu}}$ instead of $\mathcal{M}_{X}^{\text {st, } \hat{\mu}}$. Using the data $X_{i}, S_{i}, n_{i}$ of Proposition [5.10, as in (5.21) define elements $1_{X}, \mathbb{L} \in \mathcal{M}_{X}^{\text {st, } \hat{\mu}}$ by

$$
\begin{aligned}
1_{X} & =\sum_{i \in I}\left[\mathrm{GL}\left(n_{i}, \mathbb{K}\right)\right]^{-1} \otimes\left[S_{i}, \pi_{i}, \hat{\iota}\right], \\
\mathbb{L} & =\sum_{i \in I}\left[\mathrm{GL}\left(n_{i}, \mathbb{K}\right)\right]^{-1} \otimes\left[\mathbb{A}^{1} \times S_{i}, \pi_{i} \circ \pi_{S_{i}}, \hat{\imath}\right] .
\end{aligned}
$$

These are independent of choices, and $1_{X}$ is the identity in $\mathcal{M}_{X}^{\text {st, } \hat{\mu}}$.

Let $\phi: X \rightarrow Y$ be a 1-morphism of Artin $\mathbb{K}$-stacks. Define the pushforwards $\phi_{*}: K_{0}^{\hat{\mu}}\left(\operatorname{Sch}_{X}\right) \rightarrow K_{0}^{\hat{\mu}}\left(\operatorname{Sch}_{Y}\right)$ and $\phi_{*}: \mathcal{M}_{X}^{\text {st, } \hat{\mu}} \rightarrow \mathcal{M}_{Y}^{\text {st, } \hat{\mu}}$ by the analogue of (5.5). If $\phi$ is representable in $\mathbb{K}$-schemes we may also define pullbacks $\phi^{*}$ : $K_{0}^{\hat{\mu}}\left(\operatorname{Sch}_{Y}\right) \rightarrow K_{0}^{\hat{\mu}}\left(\operatorname{Sch}_{X}\right)$ and $\phi^{*}: \mathcal{M}_{Y}^{\text {st, }, \hat{\mu}} \rightarrow \mathcal{M}_{X}^{\text {st, } \hat{\mu}}$ by the analogue of (5.6). If $\phi$ is not representable in $\mathbb{K}$-schemes, we can still define $\phi^{*}: \mathcal{M}_{Y}^{\text {st, }, \hat{\mu}} \rightarrow \mathcal{M}_{X}^{\text {st, } \hat{\mu}}$ by the analogue of (5.22). Pushforwards and pullbacks have the usual functoriality properties, and the analogue of (5.7) holds for 2-Cartesian squares in Art $_{\mathbb{K}}$.

As for (5.10), there are natural morphisms of commutative rings

$$
\begin{aligned}
i_{X}: K_{0}\left(\operatorname{Sch}_{X}\right) & \longrightarrow K_{0}^{\hat{\mu}}\left(\operatorname{Sch}_{X}\right), & i_{X}: \mathcal{M}_{X}^{\text {st }} & \longrightarrow \mathcal{M}_{X}^{\text {st, } \hat{\mu}}, \\
\Pi_{X}: K_{0}^{\hat{\mu}}\left(\operatorname{Sch}_{X}\right) & \longrightarrow K_{0}\left(\operatorname{Sch}_{X}\right), & \Pi_{X}: \mathcal{M}_{X}^{\text {st, }, \hat{\mu}} & \longrightarrow \mathcal{M}_{X}^{\text {st }},
\end{aligned}
$$

given by $i_{X}:[R, \rho] \mapsto[R, \rho, \hat{\iota}]$ and $\Pi_{X}:[R, \rho, \hat{r}] \mapsto[R, \rho]$. If $X$ is a $\mathbb{K}$-scheme, there is a natural projection $\mathcal{M}_{X}^{\hat{\mu}} \rightarrow \mathcal{M}_{X}^{\text {st, }, \hat{\mu}}$.

The analogue of Definition [5.3, defining another associative, commutative product ' $\odot$ ' on $K_{0}^{\hat{\mu}}\left(\mathrm{Sch}_{X}\right), \mathcal{M}_{X}^{\text {st, } \hat{\mu}}$ and an external version ' $\square$ ', works essentially without change. For the analogue of Definition 5.4, following (5.13) we define $\mathbb{L}^{1 / 2}$ in $\mathcal{M}_{X}^{\text {st, } \hat{\mu}}$ only by $\mathbb{L}^{1 / 2}=1_{X} \boxminus \mathbb{L}_{\mathbb{K}}^{1 / 2} \in \mathcal{M}_{X}^{\text {st, } \hat{\mu}}$, where $1_{X}$ is as in (5.23), and $\mathbb{L}_{\mathbb{K}}^{1 / 2} \in \mathcal{M}_{\mathbb{K}}^{\text {st, }, \hat{\mu}}$ as in (5.12). Then $\mathbb{L}^{1 / 2} \odot \mathbb{L}^{1 / 2}=\mathbb{L}$ in $\mathcal{M}_{X}^{\text {st, }, \hat{\mu}}$, and we can define $\mathbb{L}^{n / 2}$ in $\mathcal{M}_{X}^{\text {st, } \hat{\mu}}$ for all $n \in \mathbb{Z}$ in the obvious way.

Here is the stack analogue of Definition 5.5

Definition 5.13. For each Artin $\mathbb{K}$-stack $Y$, define $I_{Y}^{\text {st, }, \hat{\mu}}$ to be the ideal in the commutative ring $\left(\mathcal{M}_{Y}^{\text {st }, \hat{\mu}}, \odot\right)$ generated by elements $\phi_{*}\left(\Upsilon^{\text {st }}\left(P \otimes_{\mathbb{Z} / 2 \mathbb{Z}} Q\right)-\right.$ $\left.\Upsilon(P)^{\text {st }} \odot \Upsilon^{\text {st }}(Q)\right)$ for all 1-morphisms $\phi: X \rightarrow Y$ with $X$ a $\mathbb{K}$-scheme and principal $\mathbb{Z} / 2 \mathbb{Z}$-bundles $P, Q \rightarrow X$, where $\Upsilon^{\text {st }}(P), \Upsilon^{\text {st }}(Q), \Upsilon^{\text {st }}\left(P \otimes_{\mathbb{Z} / 2 \mathbb{Z}} Q\right)$ are 
the images in $\mathcal{M}_{X}^{\text {st, } \hat{\mu}}$ of the elements $\Upsilon(P), \Upsilon(Q), \Upsilon\left(P \otimes_{\mathbb{Z} / 2 \mathbb{Z}} Q\right)$ in $\mathcal{M}_{X}^{\hat{\mu}}$ from Definition 5.5. Define $\overline{\mathcal{M}}_{Y}^{\mathrm{st}, \hat{\mu}}=\mathcal{M}_{Y}^{\mathrm{st}, \hat{\mu}} / I_{Y}^{\mathrm{st}, \hat{\mu}}$ to be the quotient, as a commutative ring with multiplication ' $\odot$ ', with projection $\Pi_{Y}^{\hat{\mu}}: \mathcal{M}_{Y}^{\hat{\mu}} \rightarrow \overline{\mathcal{M}}_{Y}^{\hat{\mu}}$.

The second multiplication '.', external product $\otimes$, and projection $\Pi_{Y}$ : $\mathcal{M}_{Y}^{\text {st, } \hat{\mu}} \rightarrow \mathcal{M}_{Y}^{\text {st }}$ on $\mathcal{M}_{Y}^{\text {st, } \hat{\mu}}$ do not descend to $\overline{\mathcal{M}}_{Y}^{\text {st, } \hat{\mu}}$. The other structures $\odot$, $\square, 1_{Y}, \mathbb{L}, \phi_{*}, \phi^{*}, i_{Y}, \mathbb{L}^{1 / 2}$ do descend to $\overline{\mathcal{M}}_{Y}^{\text {st, } \hat{\mu}}$. If $X$ is a $\mathbb{K}$-scheme, we have a natural projection $\overline{\mathcal{M}}_{X}^{\hat{\mu}} \rightarrow \overline{\mathcal{M}}_{X}^{\text {st, } \hat{\mu}}$. So in particular, the motives $M F_{X, s} \in \overline{\mathcal{M}}_{X}^{\hat{\mu}}$ in Theorem 5.7 also make sense in $\overline{\mathcal{M}}_{X}^{\text {st, } \hat{\mu}}$. We will use this in Theorem 5.14.

\subsection{The main result}

Here is the main result of this section, the analogue of Theorem 5.7 from [5]. The proof uses our previous results from [5, 15, the theory of rings of motives $\overline{\mathcal{M}}_{X}^{\text {st }, \hat{\mu}}$ on Artin stacks $X$ from $\$ 5.3$ and two new ingredients: Proposition $[5.8$ which says that the motives $M F_{X, s}$ from [5, Th. 5.10] pull back as one would expect under smooth morphisms of d-critical loci, and Proposition 5.19] which is a cunning trick to get round the fact that motives do not have descent in the smooth topology, that is, we do not have a motivic analogue of Theorem 4.2 .

Theorem 5.14. Let $(X, s)$ be an oriented d-critical stack, with orientation $K_{X, s}^{1 / 2}$, where $X$ is assumed of finite type and locally a global quotient. Then there exists a unique motive $M F_{X, s} \in \overline{\mathcal{M}}_{X}^{\text {st, }, \hat{\mu}}$ such that if $T$ is a finite type $\mathbb{K}$-scheme and $t: T \rightarrow X$ is smooth of relative dimension $n$, so that $(T, s(T, t))$ is an algebraic d-critical locus over $\mathbb{K}$ with natural orientation $K_{T, s(T, t)}^{1 / 2}$ as in Lemma 3.17, then

$$
t^{*}\left(M F_{X, s}\right)=\mathbb{L}^{n / 2} \odot M F_{T, s(T, t)} \quad \text { in } \overline{\mathcal{M}}_{T}^{\mathrm{st}, \hat{\mu}},
$$

where $M F_{T, s(T, t)} \in \overline{\mathcal{M}}_{T}^{\text {st, }, \hat{\mu}}$ is as in Theorem [5.7, projected from $\overline{\mathcal{M}}_{T}^{\hat{\mu}}$ in $\$ 5.1$ to $\overline{\mathcal{M}}_{T}^{\mathrm{st}, \hat{\mu}}$ in $\$ 5.3$, and $t^{*}: \overline{\mathcal{M}}_{X}^{\mathrm{st}, \hat{\mu}} \rightarrow \overline{\mathcal{M}}_{T}^{\mathrm{st}, \hat{\mu}}$ is the pullback.

We discuss how to relax the assumptions in Theorem [5.14 that $X$ is of finite type, and locally a global quotient.

Remark 5.15. (a) Let $X$ be an Artin $\mathbb{K}$-stack locally of finite type (but not necessarily of finite type), with affine geometric stabilizers. Then one can define motivic rings $K_{0}\left(\mathrm{Sch}_{X}\right), \mathcal{M}_{X}^{\mathrm{st}}, K_{0}^{\hat{\mu}}\left(\mathrm{Sch}_{X}\right), \mathcal{M}_{X}^{\mathrm{st}, \hat{\mu}}, \overline{\mathcal{M}}_{X}^{\mathrm{st}, \hat{\mu}}$ generalizing those in \$5.3. using the idea of 'local stack functions' $\operatorname{LSF}(X)$ from Joyce [14, Def. 3.9].

Elements of $K_{0}\left(\mathrm{Sch}_{X}\right)$ are $\sim$-equivalence classes of sums $\sum_{i \in I} c_{i}\left[R_{i}, \rho_{i}\right]$ for $I$ a possibly infinite indexing set, $R_{i}$ a $\mathbb{K}$-scheme locally of finite type, $\rho_{i}: R_{i} \rightarrow X$ a finite type 1-morphism, and $c_{i} \in \mathbb{Z}$ for $i \in I$, such that for any finite type $\mathbb{K}$ substack $Y \subseteq X$, we have $R_{i} \times_{X} Y \neq \emptyset$ for only finitely many $i \in I$. We set $\sum_{i \in I} c_{i}\left[R_{i}, \rho_{i}\right] \sim \sum_{j \in J} d_{j}\left[S_{j}, \sigma_{j}\right]$ if for all finite type $Y \subseteq X$, we have $\sum_{i \in I} c_{i}\left[R_{i} \times_{\rho_{i}, X, \text { inc }} Y, \pi_{Y}\right]=\sum_{j \in J} d_{j}\left[S_{j} \times_{\sigma_{j}, X \text {,inc }} Y, \pi_{Y}\right]$ in $K_{0}\left(\operatorname{Sch}_{Y}\right)$, where $K_{0}\left(\mathrm{Sch}_{Y}\right)$ is as in $\$ 5.3$ as $Y$ is of finite type. 
Then pushforwards $\phi_{*}$ on $K_{0}\left(\mathrm{Sch}_{X}\right), \mathcal{M}_{X}^{\text {st }}, \ldots$ can be defined only if $\phi: X \rightarrow$ $Y$ is a finite type 1-morphism, but pullbacks $\phi^{*}$ can be defined for arbitrary $\phi$ (requiring $\phi$ representable in $\mathbb{K}$-schemes for $K_{0}\left(\operatorname{Sch}_{X}\right), K_{0}^{\hat{\mu}}\left(\operatorname{Sch}_{X}\right)$ ).

As discussed in [5, Rem. 5.11] for $\mathbb{K}$-schemes, it is now easy to generalize Theorem 5.14 to d-critical stacks $(X, s)$ which are locally of finite type rather than of finite type, giving a unique $M F_{X, s} \in \overline{\mathcal{M}}_{X}^{\text {st, } \hat{\mu}}$ satisfying (5.24), where it is enough to consider only finite type $\mathbb{K}$-schemes $T$. Note that we cannot push $M F_{X, s}$ forward to $\overline{\mathcal{M}}_{\mathbb{K}}^{\text {st, } \hat{\mu}}$ if $X$ is not of finite type, since $\pi: X \rightarrow$ Spec $\mathbb{K}$ is not a finite type 1-morphism, and $\pi_{*}: \overline{\mathcal{M}}_{X}^{\text {st, } \hat{\mu}} \rightarrow \overline{\mathcal{M}}_{\mathbb{K}}^{\text {st, } \hat{\mu}}$ is not defined.

(b) The assumption in Theorem 5.14 that $X$ is locally a global quotient is used to prove Proposition 5.19 in $\$ 5.5$. We would have preferred to make the weaker assumption that $X$ has affine geometric stabilizers.

The issue is this: we want to characterize $M F_{X, s} \in \overline{\mathcal{M}}_{X}^{\text {st, } \hat{\mu}}$ by prescribing $t^{*}\left(M F_{X, s}\right) \in \overline{\mathcal{M}}_{T}^{\text {st, } \hat{\mu}}$ whenever $T$ is a $\mathbb{K}$-scheme and $t: T \rightarrow X$ is a smooth 1-morphism. However, if $X$ is not locally a global quotient, it seems conceivable this may not determine $M F_{X, s}$ uniquely, as there might exist $0 \neq M \in \overline{\mathcal{M}}_{X}^{\text {st, } \hat{\mu}}$ with $t^{*}(M)=0$ for all such $t: T \rightarrow X$.

One way to fix this might be to expand our whole set-up to include a suitable class of formal schemes, and then prescribe $t^{*}\left(M F_{X, s}\right)$ when $T$ is a formal scheme and $t: T \rightarrow X$ a smooth 1-morphism. If $X$ has affine geometric stabilizers, there should be enough such $t: T \rightarrow X$ to determine $M F_{X, s}$ uniquely.

Combining Theorems 2.10, 3.18, 5.14 and Corollary 3.19, and noting as in $\$ 5.1$ that moduli stacks of coherent sheaves are locally global quotients, yields:

Corollary 5.16. Let $(\boldsymbol{X}, \omega)$ be a-1-shifted symplectic derived Artin $\mathbb{K}$-stack in the sense of Pantev et al. [30, and $X=t_{0}(\boldsymbol{X})$ the associated classical Artin $\mathbb{K}$-stack, assumed of finite type and locally a global quotient. Suppose we are given a square root $\left.\operatorname{det}\left(\mathbb{L}_{\boldsymbol{X}}\right)\right|_{X} ^{1 / 2}$ for $\left.\operatorname{det}\left(\mathbb{L}_{\boldsymbol{X}}\right)\right|_{X}$. Then we may define a natural motive $M F_{\boldsymbol{X}, \omega} \in \overline{\mathcal{M}}_{X}^{\mathrm{st}, \hat{\mu}}$, which is characterized by the fact that given a diagram

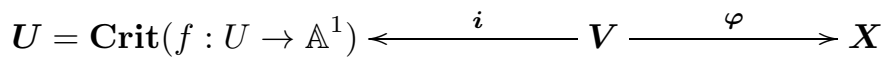

such that $U$ is a smooth $\mathbb{K}$-scheme, $\boldsymbol{\varphi}$ is smooth of dimension $n, \mathbb{L}_{\boldsymbol{V} / \boldsymbol{U}} \simeq$ $\mathbb{T}_{\boldsymbol{V} / \boldsymbol{X}}[2], \boldsymbol{\varphi}^{*}\left(\omega_{\boldsymbol{X}}\right) \sim \boldsymbol{i}^{*}\left(\omega_{\boldsymbol{U}}\right)$ for $\omega_{\boldsymbol{U}}$ the natural -1 -shifted symplectic structure on $\boldsymbol{U}=\boldsymbol{C r i t}\left(f: U \rightarrow \mathbb{A}^{1}\right)$, and $\varphi^{*}\left(\left.\operatorname{det}\left(\mathbb{L}_{\boldsymbol{X}}\right)\right|_{X} ^{1 / 2}\right) \cong i^{*}\left(K_{U}\right) \otimes \Lambda^{n} \mathbb{T}_{\boldsymbol{V} / \boldsymbol{X}}$, then $\varphi^{*}\left(M F_{\boldsymbol{X}, \omega}\right)=\mathbb{L}^{n / 2} \odot i^{*}\left(M F_{U, f}^{\mathrm{mot}, \phi}\right)$ in $\overline{\mathcal{M}}_{V}^{\mathrm{st}, \hat{\mu}}$.

Corollary 5.17. Let $Y$ be a Calabi-Yau 3-fold over $\mathbb{K}$, and $\mathcal{M}$ a finite type classical moduli $\mathbb{K}$-stack of coherent sheaves in $\operatorname{coh}(Y)$, with natural obstruction theory $\phi: \mathcal{E}^{\bullet} \rightarrow \mathbb{L}_{\mathcal{M}}$. Suppose we are given a square root $\operatorname{det}\left(\mathcal{E}^{\bullet}\right)^{1 / 2}$ for $\operatorname{det}\left(\mathcal{E}^{\bullet}\right)$. Then we may define a natural motive $M F_{\mathcal{M}} \in \overline{\mathcal{M}}_{\mathcal{M}}^{\text {st, } \hat{\mu}}$.

Corollary 5.17 is relevant to Kontsevich and Soibelman's theory of motivic Donaldson-Thomas invariants [18. Our square root $\operatorname{det}\left(\mathcal{E}^{\bullet}\right)^{1 / 2}$ roughly coincides with their orientation data [18, §5]. In [18, §6.2], given a finite type moduli stack $\mathcal{M}$ of coherent sheaves on a Calabi-Yau 3-fold $Y$ with orientation data, 
they define a motive $\int_{\mathcal{M}} 1$ in a ring $D^{\mu}$ isomorphic to our $\overline{\mathcal{M}}_{\mathbb{K}}^{\text {st, } \hat{\mu}}$. We expect this should agree with $\pi_{*}\left(M F_{\mathcal{M}}\right)$ in our notation, with $\pi: \mathcal{M} \rightarrow$ Spec $\mathbb{K}$ the projection. This $\int_{\mathcal{M}} 1$ is roughly the motivic Donaldson-Thomas invariant of $\mathcal{M}$. Their construction involves expressing $\mathcal{M}$ near each point in terms of the critical locus of a formal power series. Kontsevich and Soibelman's constructions were partly conjectural, and our results may fill some gaps in their theory.

Example 5.18. As in [14, Def. 2.1], an algebraic $\mathbb{K}$-group $G$ is called special if every étale locally trivial principal $G$-bundle over a $\mathbb{K}$-scheme is Zariski locally trivial. Any special $\mathbb{K}$-group can be embedded as a closed $\mathbb{K}$-subgroup $G \subseteq$ $\mathrm{GL}(n, \mathbb{K})$, and then $\mathrm{GL}(n, \mathbb{K}) \rightarrow \mathrm{GL}(n, \mathbb{K}) / G$ is a Zariski locally trivial principal $G$-bundle, so taking motives in $\mathcal{M}_{\mathbb{K}}^{\text {st }}$ gives $[\operatorname{GL}(n, \mathbb{K})]=[G] \cdot[\operatorname{GL}(n, \mathbb{K}) / G]$. Hence $[G]$ is invertible in $\mathcal{M}_{\mathbb{K}}^{\text {st }}$, with $[G]^{-1}=[\operatorname{GL}(n, \mathbb{K}) / G] \cdot[\operatorname{GL}(n, \mathbb{K})]^{-1}$.

Some examples of special $\mathbb{K}$-groups are $\mathbb{G}_{m}, \operatorname{GL}(n, \mathbb{K}), \operatorname{SL}(n, \mathbb{K}), \operatorname{Sp}(2 n, \mathbb{K})$, and the group of invertible elements $A^{\times}$of any finite-dimensional $\mathbb{K}$-algebra $A$. Products of special groups are special. Special $\mathbb{K}$-groups are always affine and connected, so nontrivial finite groups are not special.

Suppose a special $\mathbb{K}$-group $G$ of dimension $n$ acts on a finite type, oriented algebraic d-critical locus $\left(T, s^{\prime}\right)$ over $\mathbb{K}$ preserving $s^{\prime} \in H^{0}\left(\mathcal{S}_{T}^{0}\right)$ and the orientation $K_{T, s^{\prime}}^{1 / 2}$. Write $X=[T / G]$ for the quotient stack and $t: T \rightarrow X$ for the projection. Then $s^{\prime}$ descends to a unique d-critical structure $s$ on $X$ with $s^{\prime}=s(T, t)$ as in Example 3.14, and using Theorem 3.15 we also find that the orientation $K_{T, s^{\prime}}^{1 / 2}$ descends to a unique orientation $K_{X, s}^{1 / 2}$ on the d-critical stack $(X, s)$ with $\left.K_{X, s}^{1 / 2}\left(T^{\text {red }}, t^{\text {red }}\right) \cong K_{T, s^{\prime}}^{1 / 2} \otimes\left(\Lambda^{\text {top }} T_{T / X}^{*}\right)\right|_{T^{\text {red }}} ^{\otimes^{-1}}$.

Theorem 5.14 gives $M F_{X, s} \in \overline{\mathcal{M}}_{X}^{\text {st, } \hat{\mu}}$ with $t^{*}\left(M F_{X, s}\right)=\mathbb{L}^{n / 2} \odot M F_{T, s^{\prime}}$ in $\overline{\mathcal{M}}_{T}^{\text {st, }, \hat{\mu}}$. Applying $t_{*}$ and using $t_{*} \circ t^{*}(M)=[T, t, \hat{\imath}] \odot M$ for $M \in \overline{\mathcal{M}}_{X}^{\text {st, } \hat{\mu}}$ gives

$$
M F_{X, s} \odot[T, t, \hat{\imath}]=\mathbb{L}^{n / 2} \odot t_{*}\left(M F_{T, s^{\prime}}\right) .
$$

Now $t: T \rightarrow X$ is a principal $G$-bundle, and so Zariski locally trivial as $G$ is special. Therefore $[T, t, \hat{\iota}]=[G, \hat{\iota}] \square 1_{X}$, where $[G, \hat{\iota}]=i_{\mathbb{K}}([G]) \in \mathcal{M}_{\mathbb{K}}^{\text {st, } \hat{\mu}}$. As $[G]$ is invertible, so is $[G, \hat{\imath}]$. Thus multiplying (5.25) by $[G, \hat{\imath}]^{-1}$ gives

$$
M F_{X, s}=[G, \hat{\imath}]^{-1} \bullet\left(\mathbb{L}^{n / 2} \odot t_{*}\left(M F_{T, s^{\prime}}\right)\right) .
$$

\subsection{Proof of Theorem 5.14}

We begin with the following result, related to Proposition 5.10 .

Proposition 5.19. Let $X$ be a finite type Artin $\mathbb{K}$-stack which is locally a global quotient. Then we can find a stratification $X=\coprod_{j \in J} X_{j}$, for $J$ a finite set and $X_{j} \subseteq X$ a locally closed $\mathbb{K}$-substack, and 1-morphisms $\phi_{j}: S_{j} \rightarrow X$ smooth of relative dimension $n_{j}$ with $S_{j}$ a $\mathbb{K}$-scheme such that $\left[S_{j} \times_{X} X_{j}, \pi_{X_{j}}\right]$ is an invertible element of $\mathcal{M}_{X_{j}}^{\text {st }}$ for all $j \in J$.

Proof. As $X$ is finite type and locally a global quotient, there exist Zariski open $\mathbb{K}$-substacks $Y_{j} \subseteq X$ and equivalences $Y_{j} \simeq\left[S_{j} / \mathrm{GL}\left(n_{j}, \mathbb{K}\right)\right]$ for $j=1, \ldots, m$, where $S_{j}$ is a $\mathbb{K}$-scheme with a $\operatorname{GL}\left(n_{j}, \mathbb{K}\right)$-action, such that $X=Y_{1} \cup \cdots \cup Y_{m}$. 
Define $\phi_{j}: S_{j} \rightarrow X$ to be the composition $S_{j} \rightarrow\left[S_{j} / \mathrm{GL}\left(n_{j}, \mathbb{K}\right)\right] \stackrel{\sim}{\longrightarrow} Y_{j} \hookrightarrow X$. For $j=1, \ldots, m$, define a locally closed $\mathbb{K}$-substack $X_{j} \subseteq X$ by $X_{j}=Y_{j} \backslash\left(Y_{1} \cup\right.$ $\left.\cdots \cup Y_{j-1}\right)$. Set $J=\{1, \ldots, m\}$. Then $X=\coprod_{j \in J} X_{j}$ as $X=Y_{1} \cup \cdots \cup Y_{m}$.

Since $X_{j} \subseteq Y_{j}$ and $\phi_{j}: S_{j} \rightarrow Y_{j}$ is a principal $\operatorname{GL}\left(n_{j}, \mathbb{K}\right)$-bundle, we see that $\pi_{X_{j}}: S_{j} \times_{X} X_{j} \rightarrow X_{j}$ is a principal $\mathrm{GL}\left(n_{j}, \mathbb{K}\right)$-bundle, which is automatically Zariski locally trivial. Hence $\left[S_{j} \times_{X} X_{j}, \pi_{X_{j}}\right]=\left[\mathrm{GL}\left(n_{i}, \mathbb{K}\right)\right] \bullet 1_{X_{j}}$, which is invertible in $\mathcal{M}_{X_{j}}^{\text {st }}$ with inverse $\left[\mathrm{GL}\left(n_{i}, \mathbb{K}\right)\right]^{-1} \square 1_{X_{j}}$.

We now prove Theorem 5.14 Suppose first that there exists $M F_{X, s} \in \overline{\mathcal{M}}_{X}^{\text {st, } \hat{\mu}}$ such that (5.24) holds for all $t: T \rightarrow X$ smooth of dimension $n$ with $T$ a $\mathbb{K}$ scheme. Let $J, X_{j}, S_{j}, \phi_{j}, n_{j}$ be as in Proposition 5.19] and write $\iota_{j}: X_{j} \hookrightarrow X$ for the inclusion. Then we have

$$
\begin{aligned}
& M F_{X, s}=\sum_{j \in J}\left(\iota_{j}\right)_{*}\left(\iota_{j}^{*}\left(M F_{X, s}\right)\right) \\
& =\sum_{j \in J}\left(\iota_{j}\right)_{*}\left(\left[S_{j} \times_{X} X_{j}, \pi_{X_{j}}, \hat{\iota}\right]^{-1} \odot\left[S_{j} \times_{X} X_{j}, \pi_{X_{j}}, \hat{\iota}\right] \odot \iota_{j}^{*}\left(M F_{X, s}\right)\right) \\
& \left.=\sum_{j \in J}\left(\iota_{j}\right)_{*}\left(\left[S_{j} \times_{X} X_{j}, \pi_{X_{j}}, \hat{\iota}\right]^{-1} \odot \iota_{j}^{*}\left(\left[S_{j}, \phi_{j}, \hat{\iota}\right] \odot M F_{X, s}\right)\right)\right) \\
& =\sum_{j \in J}\left(\iota_{j}\right)_{*}\left(\left[S_{j} \times_{X} X_{j}, \pi_{X_{j}}, \hat{\iota}\right]^{-1}\right) \odot\left(\left(\phi_{j}\right)_{*} \circ \phi_{j}^{*}\left(M F_{X, s}\right)\right) \\
& =\sum_{j \in J}\left(\iota_{j}\right)_{*}\left(\left[S_{j} \times_{X} X_{j}, \pi_{X_{j}}, \hat{\iota}\right]^{-1}\right) \odot\left(\left(\phi_{j}\right)_{*}\left(\mathbb{L}^{n_{j} / 2} \odot M F_{S_{j}, s\left(S_{j}, \phi_{j}\right.}\right)\right),
\end{aligned}
$$

using $X=\coprod_{j \in J} X_{j}$ in the first step, $\left[S_{j} \times_{X} X_{j}, \pi_{X_{j}}\right]$ invertible in $\mathcal{M}_{X_{j}}^{\text {st }}$ so that $\left[S_{j} \times_{X} X_{j}, \pi_{X_{j}}, \hat{\imath}\right]=i_{X_{j}}\left(\left[S_{j} \times_{X} X_{j}, \pi_{X_{j}}\right]\right)$ is invertible in $\overline{\mathcal{M}}_{X_{j}}^{\text {st, } \hat{\mu}}$ in the second, $\left[S_{j} \times_{X} X_{j}, \pi_{X_{j}}, \hat{\iota}\right]=\iota_{j}^{*}\left(\left[S_{j}, \phi_{j}, \hat{\imath}\right]\right)$ and $\iota_{j}^{*}$ multiplicative for $\odot$ in the third, $\left[S_{j}, \phi_{j}, \hat{\iota}\right] \odot=\left(\phi_{j}\right)_{*} \circ \phi_{j}^{*}$ and $\left(\iota_{j}\right)_{*}\left(M \odot \iota_{j}^{*}(N)\right)=\left(\left(\iota_{j}\right)_{*} \circ \iota_{j}^{*}(M)\right) \odot N$ in the fourth, and (5.24) with $S_{j}, \phi_{j}, n_{j}$ in place of $T, t, n$ in the fifth. Equation (5.26) proves $M F_{X, s}$ in Theorem 5.14 is unique if it exists, and gives a formula for it.

Now define $M F_{X, s}$ to be the bottom line of (5.26). Suppose $t: T \rightarrow X$ is smooth of dimension $n$, with $T$ a $\mathbb{K}$-scheme. Define $T_{j}=X_{j} \times_{\iota_{j}, X, t} T \subseteq T$ and $U_{j}=S_{j} \times_{\phi_{j}, X, t} T$ for each $j \in J$. Then $T_{j}, U_{j}$ are $\mathbb{K}$-schemes as $X_{j} \hookrightarrow X$ and $S_{j} \rightarrow X$ are representable in $\mathbb{K}$-schemes, and we have 2 -Cartesian squares
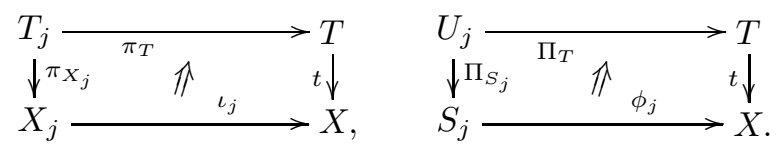

Then

$$
\begin{aligned}
& t^{*}\left(M F_{X, s}\right)=\sum_{j \in J} t^{*} \circ\left(\iota_{j}\right)_{*}\left(\left[S_{j} \times_{X} X_{j}, \pi_{X_{j}, \hat{\iota}}\right]^{-1}\right) \odot t^{*} \circ\left(\phi_{j}\right)_{*}\left(\mathbb{L}^{n_{j} / 2} \odot M F_{S_{j}, s\left(S_{j}, \phi_{j}\right)}\right) \\
& =\sum_{j \in J}\left(\pi_{T}\right)_{*} \circ \pi_{X_{j}}^{*}\left(\left[S_{j} \times_{X} X_{j}, \pi_{X_{j}}, \hat{\iota}\right]^{-1}\right) \odot\left(\Pi_{T}\right)_{*} \circ \Pi_{S_{j}}^{*}\left(\mathbb{L}^{n_{j} / 2} \odot M F_{S_{j}, s\left(S_{j}, \phi_{j}\right)}\right) \\
& =\sum_{j \in J}\left(\pi_{T}\right)_{*}\left(\left(\pi_{X_{j}}^{*}\left(\left[S_{j} \times_{X} X_{j}, \pi_{X_{j}}, \hat{\iota}\right]\right)\right){ }^{-1}\right) \odot\left(\Pi_{T}\right)_{*}\left(\mathbb{L}^{\left(n+n_{j}\right) / 2} \odot M F_{U_{j}, s\left(U_{j}, \phi_{j} \circ \Pi_{S_{j}}\right)}\right) \\
& =\sum_{j \in J}\left(\pi_{T}\right)_{*}\left(\left[S_{j} \times_{X} X_{j} \times_{X_{j}} T_{j}, \pi_{T_{j}}, \hat{\iota}\right]^{-1}\right) \odot\left(\Pi_{T}\right)_{*} \circ \Pi_{T}^{*}\left(\mathbb{L}^{n / 2} \odot M F_{T, s(T, t)}\right) \\
& =\sum_{j \in J}\left(\pi_{T}\right)_{*}\left(\left[U_{j} \times_{T} T_{j}, \pi_{T_{j}}, \hat{\iota}\right]^{-1}\right) \odot\left[U_{j}, \Pi_{T}, \hat{\iota}\right] \odot \mathbb{L}^{n / 2} \odot M F_{T, s(T, t)} \\
& \left.=\sum_{j \in J}\left(\pi_{T}\right)_{*}\left(\pi_{T}^{*}\left(\left[U_{j}, \Pi_{T}, \hat{\iota}\right]\right)^{-1} \odot \pi_{T}^{*}\left(\left[U_{j}, \Pi_{T}, \hat{\iota}\right]\right)\right) \odot \mathbb{L}^{n / 2} \odot M F_{T, s(T, t)}\right) \\
& =\sum_{j \in J}\left(\pi_{T}\right)_{*}\left(1_{T_{j}}\right) \odot \mathbb{L}^{n / 2} \odot M F_{T, s(T, t)}=\left(\sum_{j \in J}\left[T_{j}, \pi_{T}, \hat{\iota}\right]\right) \odot \mathbb{L}^{n / 2} \odot M F_{T, s(T, t)} \\
& =\left[T, \operatorname{id}_{T}, \hat{\iota}\right] \odot \mathbb{L}^{n / 2} \odot M F_{T, s(T, t)}=\mathbb{L}^{n / 2} \odot M F_{T, s(T, t)},
\end{aligned}
$$


using (5.26) and $t^{*}$ multiplicative for $\odot$ in the first step, the analogue of (5.7) for the 2-Cartesian squares (5.27) in the second, that $\pi_{X_{j}}^{*}$ is a ring morphism for $\odot$ and (5.17) for the morphism $\Pi_{S_{j}}:\left(U_{j}, s\left(U_{j}, \phi_{j} \circ \Pi_{S_{j}}\right)\right) \rightarrow\left(S_{j}, s\left(S_{j}, \phi_{j}\right)\right)$ of oriented d-critical loci which is smooth of dimension $n$ in the third, the definition of $\pi_{X_{j}}^{*}$ and (5.17) for $\Pi_{T}:\left(U_{j}, s\left(U_{j}, \phi_{j} \circ \Pi_{S_{j}}\right)\right) \rightarrow(T, s(T, t))$ smooth of dimension $n_{j}$ in the fourth, $S_{j} \times_{X} X_{j} \times_{X_{j}} T_{j} \cong S_{j} \times_{X} T_{j}=U_{j} \times_{T} T_{j}$ and $\left(\Pi_{T}\right)_{*} \circ \Pi_{T}^{*}=\left[U_{j}, \Pi_{T}, \hat{\iota}\right] \odot$ in the fifth, $\left(\pi_{T}\right)_{*}(M) \odot N=\left(\pi_{T}\right)_{*}\left(M \odot \pi_{T}^{*}(N)\right)$ in the sixth, and $T=\coprod_{j} T_{j}$ in the ninth.

Equation (5.28) proves (5.24) for all $t: T \rightarrow X$ smooth of dimension $n$ with $T$ a $\mathbb{K}$-scheme, as we want, for $M F_{X, s}$ the bottom line of (5.26). The argument of (5.26) shows $M F_{X, s}$ is unique, and is in particular independent of the choice of $J, X_{j}, S_{j}, \phi_{j}, n_{j}$ in Proposition 5.19. This completes the proof.

\section{References}

[1] A.A. Beilinson, J. Bernstein, and P. Deligne, Faisceaux pervers, Astérisque 100, 1982.

[2] E. Bouaziz and I. Grojnowski, A d-shifted Darboux theorem, arXiv:1309.2197, 2013.

[3] C. Brav, V. Bussi, D. Dupont, D. Joyce, and B. Szendrői, Symmetries and stabilization for sheaves of vanishing cycles, Journal of Singularities 11 (2015), 85-151. arXiv:1211.3259.

[4] C. Brav, V. Bussi and D. Joyce, A Darboux theorem for derived schemes with shifted symplectic structure, arXiv:1305.6302, 2013.

[5] V. Bussi, D. Joyce and S. Meinhardt, On motivic vanishing cycles of critical loci, arXiv:1305.6428, 2013.

[6] J. Denef and F. Loeser, Geometry on arc spaces of algebraic varieties, European Congress of Mathematics, Vol. I (Barcelona, 2000), 327-348, Progr. Math. 201, Birkhäuser, Basel, 2001. math.AG/0006050.

[7] A. Dimca, Sheaves in Topology, Universitext, Springer-Verlag, Berlin, 2004.

[8] T. Ekedahl, On the adic formalism, pages 197-218 in The Grothendieck Festschrift, vol. II, Progr. Math. 87, Birkhäuser, Boston, 1990.

[9] E. Freitag and R. Kiehl, Etale cohomology and the Weil Conjecture, Ergeb. der Math. und ihrer Grenzgebiete 13, Springer-Verlag, 1988.

[10] D. Gaitsgory and N. Rozenblyum, Crystals and $\mathscr{D}$-modules, arXiv:1111.2087, 2011.

[11] S.I. Gelfand and Y.I. Manin, Methods of Homological Algebra, second edition, Springer-Verlag, Berlin, 2003. 
[12] R. Hartshorne, Algebraic Geometry, Graduate Texts in Math. 52, Springer, New York, 1977.

[13] H. Hironaka, Resolution of singularities of an algebraic variety over a field of characteristic zero I, II, Ann. Math. 79 (1964), 109-203 and 205-326.

[14] D. Joyce, Motivic invariants of Artin stacks and 'stack functions', Quart. J. Math. 58 (2007), 345-392. math.AG/0509722.

[15] D. Joyce, A classical model for derived critical loci, to appear in Journal of Differential Geometry, 2014. arXiv:1304.4508.

[16] D. Joyce, Y. Song, A theory of generalized Donaldson-Thomas invariants, Mem. Amer. Math. Soc. 217 (2012), no. 1020. arXiv:0810.5645.

[17] R. Kiehl and R. Weissauer, Weil Conjectures, perverse sheaves and l'adic Fourier transform, Springer-Verlag, 2001.

[18] M. Kontsevich and Y. Soibelman, Stability structures, motivic DonaldsonThomas invariants and cluster transformations, arXiv:0811.2435, 2008.

[19] M. Kontsevich and Y. Soibelman, Cohomological Hall algebra, exponential Hodge structures and motivic Donaldson-Thomas invariants, Commun. Number Theory Phys. 5 (2011), 231-352. arXiv:1006.2706.

[20] A. Kresch, Cycle groups for Artin stacks, Invent. Math. 138 (1999), 495536. math.AG/9810166

[21] G. Laumon and L. Moret-Bailly, Champs algébriques, Ergeb. der Math. und ihrer Grenzgebiete 39, Springer-Verlag, Berlin, 2000.

[22] Y. Laszlo and M. Olsson, The six operations for sheaves on Artin stacks. I Publ. Math. I.H.E.S. 107 (2008), 109-168. math.AG/0512097.

[23] Y. Laszlo and M. Olsson, The six operations for sheaves on Artin stacks. II, Publ. Math. I.H.E.S. 107 (2008), 169-210. math.AG/0603680.

[24] Y. Laszlo and M. Olsson, Perverse t-structure on Artin stacks, Math. Z. 261 (2009), 737-748. math.AG/0606175.

[25] Y. Liu and W. Zheng, Enhanced six operations and base change theorem for sheaves on Artin stacks, arXiv:1211.5948, 2012.

[26] Y. Liu and W. Zheng, Enhanced adic formalism, biduality, and perverse t-structures for higher Artin stacks, preprint, 2012.

[27] E. Looijenga, Motivic measures, Séminaire Bourbaki, Vol. 1999/2000. Astérisque 276 (2002), 267-297. math.AG/0006220.

[28] D. Massey, Natural commuting of vanishing cycles and the Verdier dual, arXiv:0908.2799, 2009. 
[29] M. Olsson, Sheaves on Artin stacks, J. Reine Angew. Math. 603 (2007), $55-112$.

[30] T. Pantev, B. Toën, M. Vaquié and G. Vezzosi, Shifted symplectic structures, Publ. Math. I.H.E.S. 117 (2013), 271-328. arXiv:1111.3209.

[31] A.G.M. Paulin, The Riemann-Hilbert correspondence for algebraic stacks, arXiv:1308.5890, 2013.

[32] B. Toën, Higher and derived stacks: a global overview, pages 435-487 in Algebraic Geometry - Seattle 2005, Proc. Symp. Pure Math. 80, Part 1, A.M.S., Providence, RI, 2009. math.AG/0604504.

[33] B. Toën and G. Vezzosi, Homotopical Algebraic Geometry II: Geometric Stacks and Applications, Mem. Amer. Math. Soc. 193 (2008), no. 902. math.AG/0404373.

Address for Christopher Brav:

Institute for Advanced Study, Einstein Drive, Princeton, NJ 08540, U.S.A.

E-mail: brav@ias.edu.

Address for Oren Ben-Bassat:

Department of Mathematics, University of Haifa, Haifa, Israel.

E-mail: ben-bassat@math.haifa.ac.il. Address for Vittoria Bussi and Dominic Joyce:

The Mathematical Institute, Radcliffe Observatory Quarter, Woodstock Road, Oxford, OX2 6GG, U.K.

E-mails: bussi@maths.ox.ac.uk, joyce@maths.ox.ac.uk. 